\title{
Códigos cíclicos como ideais em álgebras de grupos
}

\author{
Valéria Ostete Jannis Luchetta
}

DISSERTAÇÃO APRESENTADA

$\mathrm{AO}$

INSTITUTO DE MATEMÁTICA E ESTATÍSTICA

DA

UNIVERSIDADE DE SÃO PAULO

PARA

OBTENÇÃO DO GRAU DE MESTRE EM

MATEMÁTICA

Área de Concentração: Álgebra
Orientador: Prof. Dr. Francisco César Polcino Milies 


\section{Códigos cíclicos como ideais em álgebras de grupos}

Este exemplar corresponde à redação final da dissertação devidamente corrigida e defendida por Valéria Ostete Jannis Luchetta

e aprovada pela comissão julgadora.

São Paulo, 26 de julho de 2005.

Banca examinadora:

Prof. Dr. Francisco César Polcino Milies (orientador) IME - USP Prof. Dr. Raul Antônio Ferraz IME - USP

Prof. Dr. Antônio Paques

UNICAMP 
aos amores de minha vida:

Sérgio e Giovanna. 


\section{Agradecimentos}

Agradeço a meu orientador professor Francisco César Polcino Milies, pela amizade que fizemos, pela dedicação, compreensão e paciência que teve durante nosso trabalho. No entanto, seu maior papel para mim foi mostrar com mestria o significado da palavra mestre.

Aos professores do IME muitíssimo obrigado por aguçar meu desejo ao conhecimento, entre eles, professor Leônidas de Oliveira Brandão, cuja amizade e incentivo me trouxeram até aqui. A professora Roseli Fernandez por suas excelentes aulas e o trabalho de iniciação cientifica, que me inspiram.

Agradeço aos professores que se tornaram meus amigos e ao qual prezo muito: professor Henrique Panzarelli e professor Jorge Aragona.

Ao professor Antônio Paques e aos amigos professor Raul Ferraz e Osnel agradeço pelo apoio, participação dos seminários e por todos os comentários, sugestões e ajuda que me deram quando começamos a estudar o assunto.

Agradeço aos amigos que fiz durante o curso de mestrado aos quais nos apoiávamos mutuamente: Andréia, Fabio, Eliza, Márcia e Antônio.

Acima de tudo, eu agradeço a minha família: Sérgio e Giovanna. Vocês foram maravilhosos durante todo este caminho, me suportaram, me apoiaram, me compreenderam e me incentivaram. Amo vocês.

Agradeço também meus pais: Lorival e Mércia, meus sogros Antônio e Isabel, minha irmã Valesca e meu cunhado Edgard, minha cunhada Maria Inês e minha tia Ivani pela torcida.

A todos que durante esta jornada cruzaram meu caminho e deixaram um pouquinho de seu conhecimento em mim, muitíssimo obrigada. 


\begin{abstract}
The aim of this work is to study cyclic codes as ideals in semisimple group algebras, building such codes from the set of primitive idempotents of this group algebra. To do so, we study some topics from the theory of error-correctory codes and, in particular, $\mathrm{BCH}$ and Reed-Solomon codes; the computation of the number of simple components in the group algebra and the construction of the set of primitive idempotents under certain restrictions that appear naturally.
\end{abstract}

\title{
Resumo
}

O objetivo deste trabalho é estudar códigos cíclicos como ideais em álgebras de grupo semisimples, construindo tais códigos a partir do conjunto de idempotentes primitivos desta álgebra de grupo. Para isso, estudaremos alguns tópicos da Teoria dos Códigos Corretores de Erros e, em particular, os códigos $\mathrm{BCH}$ e Reed-Solomon; o cálculo do número de componentes simples na álgebra de grupo e a construção do conjunto de idempotentes primitivos sob certas restrições que aparecem naturalmente. 


\section{Sumário}

Introdução $\quad$ iii

1 Preliminares Algébricos 1

1.1 Corpos Finitos . . . . . . . . . . . . . . . . . . . . . 1

1.2 Anéis Semisimples . . . . . . . . . . . . . . . . 6

1.3 Anéis de Polinômios . . . . . . . . . . . . . . . 7

1.4 Anéis de Grupos . . . . . . . . . . . . . . . . . . . . . . . . 12

1.5 Representações de Grupos . . . . . . . . . . . . . . . . . . . . . . 15

1.6 Caracteres de Grupos . . . . . . . . . . . . . . . . . . . . . . . . 16

1.7 Idempotentes . . . . . . . . . . . . . . . . . . . . . . . 22

2 O Número de Componentes Simples 25

2.1 Introdução . . . . . . . . . . . . . . . . . . . . . 25

2.2 O número de componentes simples . . . . . . . . . . . 27

3 Conceitos Básicos da Teoria de Códigos 31

3.1 Códigos Corretores de Erros . . . . . . . . . . . . . . . . . 31

3.2 Códigos Lineares . . . . . . . . . . . . . . . . . . . . . . . . . . . . . . . . . . . . . . . . . . . . . . .

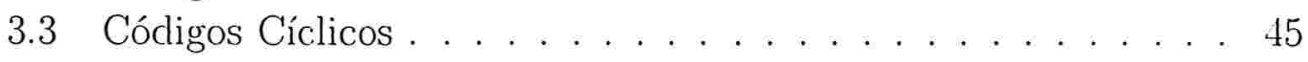

3.4 Zeros do código cíclico . . . . . . . . . . . . . . . . . . . 52

4 Códigos BCH e Reed-Solomon $\quad 57$

4.1 Códigos Bose-Chaudhuri-Hocquenghem -BCH . . . . . . . . . 57

4.2 Códigos Reed-Solomon . . . . . . . . . . . . . . . 63

5 Um caso particular 65

5.1 Idempotentes Primitivos . . . . . . . . . . . . . . . . . . 65

5.2 Determinação dos códigos BCH e Reed-Solomon . . . . . . . 68 
5.3 Apêndice . . . . . . . . . . . . . . . . . . . . . . 73

6 Códigos Cíclicos Minimais $\quad 75$

6.1 Preliminares . . . . . . . . . . . . . . 75

6.2 Códigos Minimais . . . . . . . . . . . . . . . . . . . . . 80

6.3 Apêndice . . . . . . . . . . . . . . . . . . . . . . . . . 91

7 Códigos Abelianos Minimais $\quad 101$

7.1 O Cálculo dos Idempotentes . . . . . . . . . . . . . . . . . 101

7.2 Dimensão e distância mínima . . . . . . . . . . . . . . 106

Referências Bibliográficas 110 


\section{Introdução}

A teoria dos códigos corretores cle erros é um campo amplamente pesquisado até hoje por diversas áreas do conhecimento: matemática, computação, engenharia elétrica e estatística entre outras.

$\mathrm{Na}$ década de quarenta quando os computadores eram máquinas muito caras, apenas instituições de grande porte como o governo e as universidades tinham condições de mantê-los, usando-os para executar tarefas numéricas complexas, como calcular a órbita precisa de Marte ou analisar estatisticamente o Censo [26].

O Laboratório Bell de Tecnologia possuia tais computadores e Richard W. Hamming deparou-se com estas máquinas em 1947, cujo acesso era restrito aos fins de semanas. Ele relembra

Dois finais de semanas consecutivos eu fui e, descobri que todas minhas coisas tinham sido descarregadas e nada foi feito. Eu estava realmente aborrecido e irritado porque queria estas respostas e dois finais de semanas tinham sido perdidos. E então eu disse "Maldição", se as máquinas podem detectar um erro, porque não podemos localizar a posição do erro e corrigi-lo. [9]

Esta questão foi crucial para o desenvolvimento dos códigos corretores de erros.

Nesta época as máquinas paravam seu funcionamento quando detectavam um erro e o trabalho não podia ser concluido. Assim, Hamming ficou pensando nesta questão, embora este problema não fosse novo, pois já ocorria nas centrais telefônicas e de telegrafos.

Sua pesquisa tinha como objetivo principal a trasmissão de uma cadeia de caracteres (strings) composta de 0 e 1 . Isto é devido ao fato que o sistema binário é a forma natural para representarmos dispositivos abertos e fechados de um circuito, circuitos flip-flop ${ }^{1}$, pontos e traços ou valores positivos e negativos. Assim, assumiremos que o equipamento de transmissão opera informações na linguagem binária, na forma de sequências de zeros e uns.

Uma cadeia de $m$ dígitos será chamada de palavra de comprimento $m$. Um conjunto de palavras denomina-se código. Por exemplo, podemos considerar

\footnotetext{
${ }^{1}$ Flip-flop é um tipo especial de circuito de memória, que usa dois transístores para armazenar cada bit de dados, ao invés de apenas um transistor, como na mémoria RAM comum.
} 
o código $\mathcal{C}$ formado por todas as possíveis palavras binárias de comprimento 3:

$$
\begin{array}{llll}
000 & 001 & 010 & 100 \\
011 & 101 & 110 & 111
\end{array}
$$

Se o canal de transmissão sofrer interferência, a palavra recebida pode ser diferente da palavra enviada. Por exemplo, se queremos enviar a palavra 010 pertencente ao código descrito acima e a enviamos por um canal onde ocorra um erro, então a palavra recebida pode ser, por exemplo, 011. Esta palavra pertence ao código e não será recebida como errada, pior ainda, esta palavra terá um outro significado, que poderá alterar a mensagem.

Assim, uma possível solução para que não ocorra o descrito acima seria que as palavras que constituem um código sejem bem diferentes uma das outras, para que possamos detectar e corrigir um ou mais erros.

Uma solução seria enviarmos palavras bem compridas e, é claro que, isto implicaria numa quantidade de informação grande enviada por unidade de tempo, o que acarretaria um ganho na confiabilidade, mas este ganho seria compensado por um menor fluxo de informação durante a transmissão.

Suponha que queremos enviar apenas duas informações, que representaremos pelas palavras 0 e 1 respectivamente. Vamos construir o código $\mathcal{C}=\{00$, $11\}$ formado repetindo uma vez cadla palavra a ser enviada. Este código detectará erros únicos ocorridos durante o envio da palavra, pois qualquer erro numa palavra a transformará em uma palavra que não pertence ao código. Observe que se dois erros ocorrerem durante a transmissão de uma palavra deste código, aquela será transformada em outra palavra que também pertence ao código. Por exemplo, se enviarmos a palavra 00 e dois erros ocorrem na transmissão, transformando-a em 11, esta nova palavra também pertence ao código. Portanto, este código não permite detectar dois erros.

Assim, repetindo cada palavra formada por um dígito $n-1$ vezes, nos formamos o código de repetição, que consiste de duas palavras de comprimento $n$, a palavra cujos dígitos são todos iguais a 1 e a palavra cujos dígitos são todos iguais a 0 . Este código detecta até $n-1$ erros ocorridos durante a transmissão. Neste caso, o primeiro dígito de cada palavra denomina-se dígito de informação (ou mensagem) enquanto os $n-1$ dígitos restantes são os dígitos de redundância (ou verificação). Este código, embora eficiente, permite o envio de apenas duas palavras diferentes.

Queremos um código que nos permita o envio de mais palavras, não apenas cluas e, mesmo assim, detectam erros. 
Observe que o número total de palavras de um dado comprimento $m$ é $2^{m}$. Vamos construir um código de repetição de blocos.

Uma palavra no código de repetição será formada repetindo $s$ vezes uma palavras de comprimento $m$. Portanto, este código é constituido por $2^{m}$ palavras de comprimento $n=s m$.

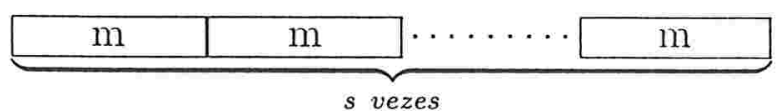

Note que, uma mensagem que se deseja transmitir é uma palavra constituida por informações de comprimento $m$, e os $s-1$ blocos restantes que constituem uma palavra no código de repetição são as redundâncias.

Por exemplo, se $m=4$ e $s=2$ podemos formar $16\left(=2^{4}\right)$ mensagens, e consequentemente, 16 palavras de comprimento $8(n=s m)$, cujos primeiros quatro dígitos coincidem com os quatro últimos.

Todas as mensagens possíveis de comprimento 4 são:

\begin{tabular}{|l||l||l||l|}
0000 & 1000 & 0110 & 1011 \\
0001 & 0011 & 1010 & 1101 \\
0010 & 0101 & 1100 & 1110 \\
0100 & 1001 & 0111 & 1111
\end{tabular} \mid

Assim, o código de repetição é formado pelas palavras:

\begin{tabular}{|l||l||l||l||}
00000000 & 10001000 & 01100110 & 10111011 \\
00010001 & 00110011 & 10101010 & 11011101 \\
00100010 & 01010101 & 11001100 & 11101110 \\
01000100 & 10011001 & 01110111 & 1111111
\end{tabular} \mid

Este código é capaz de detectar todo erro único. Por exemplo, se a palavra 11001100 foi enviada e ocorreu um erro durante a transmissão localizado no oitavo dígito, então a palavra recebida será 11001101 que não pertence ao código. Comparando o primeiro com o segundo bloco, é possível detectar que ocorreu um erro na quarta posição do bloco, mas não é possível saber em qual dos blocos ocorreu o erro.

Esta transmissão pode ser ilustrada como:

$$
11001101 \longrightarrow 11001101
$$

(A linha horizontal lê-se "é recebida como") 
Como antes dois erros não serão sempre detectados, como por exemplo no seguinte caso:

$$
11001100 \longrightarrow 11101110
$$

Obter precisão num canal de transmissão que sofra intcrferências requer dígitos de redundância nas palavras e, portanto palavras mais longas, e isto diminui o fluxo de informação. O código de repetição embora bastante confiável torna-se muito lento.

Um código formado por palavras de comprimento $n$, cujas mensagens tenham comprimento $m$ será denotado por $(n, m)$-código.

O objetivo da teoria dos códigos corretores de erros é desenvolver métodos para enviar mensagens rápidas nas quais seja possível detectar e corrigir erros.

Em canais em que poderão acontecer no máximo um erro por palavra, a concepção de "paridade" ajuda a detectar erros com rapidez e confiabilidade ao mesmo tempo.

Diz-se que uma palavra composta por dígitos binários tem paridade 0 se o número de dígitos iguais a 1 na palavra é par ou, equivalentemente, se a soma dos dígitos da palavra é igual a 0 em módulo 2. Caso contrário, a palavra tem paridade 1 .

Com o auxílio da verificação de paridade podemos desenvolver um código eficiente capaz de detectar um erro único.

Por exemplo, consideremos palavras de comprimento quatro, logo podemos formar $16\left(=2^{4}\right)$ palavras. Anexaremos um quinto dígito, no final da palavra, de modo que a palavra formada tenha comprimento 5 e paridade 0 . Portanto, formamos um código composto pelas palavras:

$$
\begin{array}{|l||l||l||l|}
00000 & 10001 & 01100 & 10111 \\
00011 & 00110 & 10100 & 11011 \\
00101 & 01010 & 11000 & 11101 \\
01001 & 10010 & 01111 & 11110
\end{array} \mid
$$

Qualquer erro único na transmissão de uma palavra faz a paridade desta ficar igual a 1 , o que nos permite detectar o erro.

Por exemplo, se recebermos a palavra 11010 sabemos que um erro ocorreu, rlescle que $1+1+0+1+0 \equiv 1 \quad(\bmod 2)$. Mas, se ocorrerem dois erros isto escapa da detecção, uma vez que a paridade da palarra recebicla é novamente 0 . 
Por volta de 1940, no Laboratório Bell de Telefonia usavam um código similar ao $(5,4)$-código de verificação de paridade que corrigia um erro único, mas adiante este códligo foi aprimorado.

As comunicações entre máquinas e entre seus componentes internos estão sujeitos a interferências internas e externas. Hamming interessou-se pelos erros que ocorriam internamento nos computadores e desenvolveu um código corretor de erro único e códigos que detectam até dois erros e corrigem erro único, mas somente em abril de 1950 seu trabalho foi publicado no "The Bell System Technical Journal"[10] (A publicação tardia deste artigo ocorreu devido ao pedido de patente destes códigos matemáticos que o Laboratório Bell fez).

Durante os três anos de elaboração destes códigos e da publicação de seu trabalho, Hamming publicou alguns memorandos conforme sua pesquisa evoluía.

Ele queria fazer um código mais eficiente e indagou se era possível construir um código corretor de erro único onde as palavras teriam quatro dígitos de informações e menos do que oito dígitos de redundâncias por palavra. Esta questão foi respondida indiretamente em outubro de 1948, por C. E. Shannon em seu artigo intitulado "A Mathematical Theory of Communication", publicado no "The Bell System Technical Journal"[20]. O artigo de C. E. Shannon deu início a um novo campo da engenharia elétrica, a Teoria da Informação, cuja enfase era o estudo do canal de comunicação que recebia interferência durante as transmissões de dados, e um ramo dela chamou-se códigos corretores de erros. A partir deste artigo, podemos dizer, que houve um desenvolvimento contínuo e bastante significativo da Teoria dos Códigos.

Em seu artigo, Shannon enfatiza que o problema fundamental cla comunicação é a reprodução exata de cada caracter do modo como este foi enviado, pois cada mensagem tem significado próprio.

O esquema de representação de um sistema de comunicação que Shannon propõe em seu trabalho é utilizado até hoje e, iremos reproduzi-lo a seguir :

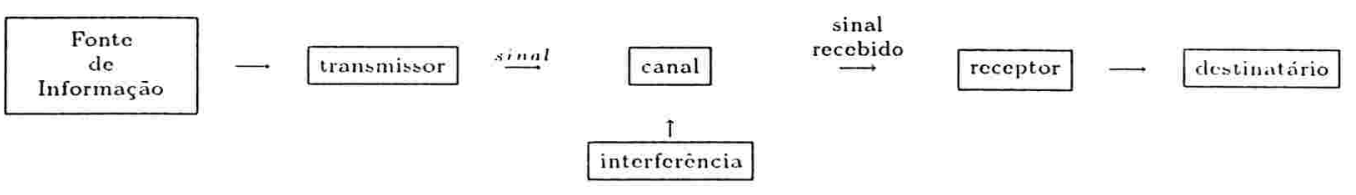

Este esquema consiste essencialmente de cinco partes:

(1) Fonte de Informação que produz uma mensagem ou sequência do 
mensagens para serem transmitidas a um terminal receptor. A mensagem pode ser de vários tipos: (a) Uma sequência de símbolos como no sistema de telegrafos; (b) Uma função de tempo $f(t)$ como em rádio ou telefonia; (c) Uma função de tempo e outras variáveis como em televisão branca e preta; (d) Duas ou mais funções, digamos $f(t), g(t), h(t)$ - neste caso como em transmissão de som; (d) Várias funções de várias variáveis - em televisões coloridas.

(2) Um transmissor ou codificador que opera a mensagem produzindo um sinal para transmissão sobre um canal. Em telefonia esta operação consiste apenas em transformar a pressão do som em uma corrente elétrica proporcional. Em telegrafia temos uma operação de codificação que reproduz uma sequência de pontos, traços e espaços que correspondem a mensagem.

(3) O canal é apenas o meio usado para transmitir o sinal do transmissor para o receptor. Ele pode ser um par de fios, cabos coaxiais, um canal de radiofrequência, circuitos integrados, um feixe de luz, etc.

(4) O receptor ou decodificador normalmente desempenha a operação inversa feita pelo transmissor, reconstruindo a mensagem.

(5) O destinatário é a pessoa (ou objeto) para quem a mensagem é destinada.

Marcel J. E. Golay que trabalhava no Signal Corps Enginneering Laboratories at Fort Monmouth, em Nova Jersey, leu a descrição do (7, 4)-código de Hamming no artigo de Shannon em 1948, e estendeu o resultado para código corretor de erro único de comprimento primo $p$. Seu trabalho foi publicado em julho de 1949 em Proceedings of the I. R. E. (I. E. E. E.), o artigo foi intitulado "Notes on Digital Coding".

Ainda com base neste artigo, Golay desenvolveu os hoje chamados $(23,12)$ e $(11,6)$ códigos de Golay. Posteriormente desenvolveu o $(24,8)$-código de Golay que foi usado pela espaçonave Voyager para transmitir fotografias coloridas de Júpiter e Saturno.

Golay, Hamming ou Shannon, quem foi o criador da teoria dos códigos? Esta é uma questão muito discuticla ainda, mas o fato que nos interessa é que estes foram os grandes nomes que começaram a trabalhar com este assunto e desenvolverem estudos e teorias que são usadas até hoje no nosso dia a dia. 
como por exemplo: na comunicação móvel (telefones celular), nos aparelhos de armazenamentos de dados (gravador, compact disk, DVD), além de na comunicação via satélite, processamento de imagens digitais, proteção de mémoria SRAM (células de mémoria estática), internet e rádio, entre outras.

Recentemente Robert F. Rice e Marvin Perlman receberam prêmios concediclos pela $\mathrm{NASA}^{2}$ por suas invenções e contribuições; suas descobertas tiveram um significante impacto nos programas espaciais e na economia dos EUA. Rice, que era membro do comando de vôo do $\mathrm{JPL}^{3}$ e da seção de administração de sistemas de dados, desenvolveu um sistema de compressão de dados digitais, trabalhou na codificação protegida de erros, desenvolveu e promoveu uma concepção chamada Advanced Imaging Communication System (AICS) no início dos anos 70. Este sistema promoveu uma enorme melhoria na eficiência das telecomunicações por combinar uma sofisticada compressão de dados com técnicas de codificação com correção de erros. O sistema combina técnicas chamada de codificação Reed-Solomon (que estudaremos no capítulo IV) com o método padrão existente na época. Esta técnica ficou conhecida como algoritmo Rice, o AICS.

Perlman, um empregado aposentado e colega de seção de Rice, trabalhou na codificação algébrica com correção de erros, na decodificação da comunicação espacial, e no sistema de criptografia para comunicação segura. Além disso, ele formulou métodos matemáticos que tornaram viáveis a decodificação usada em toda missão espacial futura, que se adaptava ao sistema de codificação padrão.

Ambos, Rice e Perlman trabalharam ajudando a NASA/ JPL no envio de fotografias e outros dados das espaçonaves planetárias para a Terra.

O algoritmo Rice foi aplicado na espaçonave Voyager 2 para Urano e $\mathrm{Ne}$ tuno em 1986 e 1989. Atualmente, estes códigos são amplamente utilizados em programas espaciais da NASA e do JPL, por exemplo na missão Galileo para Júpiter, na missão Cassini para Saturno e na recente missão a Marte.

Nosso trabalho tem como objetivo o estudo dos códigos cíclicos minimais e, para isto começaremos relembrando alguns tópicos matemáticos que usaremos ao longo do texto. Este será nosso Capítulo I. No Capítulo II, desenvolveremos o cálculo do número de componentes simples da álgebra de grupo de um grupo abeliano finito segundo [7]. No Capítulo III intro-

\footnotetext{
${ }^{2} \mathrm{NASA}=$ National Aeronautics and Space Administration

${ }^{3} \mathrm{JPL}=$ Jet Propulsion Laboratory
} 
duziremos conceitos básicos da Teoria cle códigos, onde daremos definições, propriedades e exemplos de códigos corretores de erros, códigos lineares e códigos cíclicos; apresentaremos estes dois últimos tópicos de modo ligeiramente diferente dos encontrados na literatura desta teoria.

No Capítulo IV, daremos ênfase aos códigos BCH e códigos Reed-Solomon e, nos Capítulos V, VI e VII descreveremos os códigos cíclicos minimais através de elementos idempotentes primitivos sob algumas restrições: no Capítulo V descreveremos os códigos cíclicos num caso particular e, nestas condições determinaremos os códigos BCH e Reed-Solomon; no Capítulo VI estudaremos códigos cíclicos minimais, no caso em que $G$ é um grupo cíclico finito de ordem $p^{n}$ e $2 p^{n}$, onde $p$ é primo; no Capítulo VII estenderemos os resultados apresentados no Capítulo VI e calcularemos a dimensão e distância mínima destes.

Observemos que neste trabalho estamos considerando apenas canais simétricos, isto é, canais que possuem as seguintes propridades:

- Todos os símbolos transmitidos têm a mesma probabilidade (pequena) de serem recebidos errados;

- Se um símbolo é recebido errado, a probabilidade de ser qualquer um dos outros é a mesma. 


\section{Capítulo 1}

\section{Preliminares Algébricos}

Como já foi dito anteriormente, nosso objetivo é o estudo de códigos cíclicos mínimais usando álgebras de grupo finito sobre corpos finitos.

Neste capítulo enunciaremos lemas, proposições e teoremas à respeitos destes tópicos.

\subsection{Corpos Finitos}

Sejam $\mathbb{F}$ e $\mathbb{K}$ corpos. Escreveremos $\mathbb{K} \backslash \mathbb{F}$ para indicar que $\mathbb{K}$ é uma extensão de $\mathbb{F}$. Neste caso, $\mathbb{K}$ é também um espaço vetorial sobre $\mathbb{F}$. Se a dimensão de $\mathbb{K}$ sobre $\mathbb{F}$ é finita, dizemos que $\mathbb{K}$ é uma extensão finita de $\mathbb{F}$ e denotaremos esta dimensão por $[\mathbb{K}: \mathbb{F}]$.

Utilizaremos como bibliografia básica para esta seção [13, capítulo II].

Teorema 1.1.1. Seja $\mathbb{F}$ um corpo finito. Então $\mathbb{F}$ tem $p^{n}$ elementos, onde o primo $p$ é a característica de $\mathbb{F}$ e n é o grau de $\mathbb{F}$ sobre seu subcorpo primo.

Lema 1.1.2. Se $\mathbb{F}$ é um corpo finito com $q$ elementos então todo elemento $v \in \mathbb{F}$ satisfaz $v^{q}=v$.

Lema 1.1.3. Se $\mathbb{F}$ é um corpo finito com $q$ elementos $e \mathbb{F}_{p}$ é o subcorpo primo de $\mathbb{F}$ então o polinômio $X^{q}-X \in \mathbb{F}_{p}[X]$ fatora-se em $\mathbb{F}[X]$ como

$$
X^{q}-X=\prod_{b \in \mathbb{F}}(X-b)
$$

e. F é um corpo de decomposição de $X^{q}-X$ sobre $\mathbb{F}_{p}$. 
Teorema 1.1.4 (Existência e Unicidade de Corpos Finitos). Para qualquer primo $p$ e para qualquer inteiro positivo $n$ existe um corpo finito com $p^{n}$ elementos. Qualquer corpo finito com $q=p^{n}$ elementos é isomorfo ao corpo de decomposição do polinômio $f_{q}(X)=X^{q}-X$ sobre $\mathbb{F}_{p}$.

A partir de agora, $\mathbb{F}_{q}$ denotará sempre o corpo com $q$ elementos.

Para um corpo finito $\mathbb{F}_{q}$ denotaremos por $\mathbb{F}_{q}^{*}$ o grupo multiplicativo de elementos não nulos, de $\mathbb{F}_{q}$.

Teorema 1.1.5. Para todo corpo finito $\mathbb{F}_{q}$ o grupo multiplicativo $\mathbb{F}_{q}^{*}$ de elementos não nulos, de $\mathbb{F}_{q}$ é cíclico.

Definição 1.1.6. Todo elemento de $\mathbb{F}_{q}$ que gera o grupo cíclico $\mathbb{F}^{*}$ diz-se elemento primitivo de $\mathbb{F}_{q}$.

Se $\zeta$ é um elemento primitivo de $\mathbb{F}_{q}$ então

$$
\mathbb{F}_{q}=\left\{0,1, \zeta, \ldots, \zeta^{q-2}\right\}
$$

Note que, se $\mathbb{F}_{p}$ é o corpo primo de $\mathbb{F}_{q}$ então $\mathbb{F}_{q}=\mathbb{F}_{p}(\zeta)$ e $\zeta$ é um elemento primitivo de $\mathbb{F}_{q}$ sobre $\mathbb{F}_{p}$, no sentido usual da Teoria de corpos.

Teorema 1.1.7. Sejam $\mathbb{F}_{q}$ um corpo finito e $\mathbb{K}$ uma extensão finita de $\mathbb{F}_{q}$. Então $\mathbb{K}$ é uma extensão algébrica simples.

Corolário 1.1.8. Para todo corpo finito $\mathbb{F}_{q}$ e para todo inteiro positivo $n$ existe um polinômio irredutivel de $\mathbb{F}_{q}[X]$ de grau $n$.

Mais adiante, em nosso trabalho utilizaremos polinômios sobre corpos finitos. Assim, introduziremos algumas propriedades que usaremos futuramente.

Teorema 1.1.9. Se $f(X)$ é um polinômio irredutível de $\mathbb{F}_{q}[X]$ de grau $m$ então $f$ tem uma raiz $\xi$ em $\mathbb{F}_{q^{m}}$. Além disso, todas as raízes de $f$ são simples e são dadas pelos $m$ elementos distintos $\xi, \xi^{q}, \xi^{q^{2}}, \ldots, \xi^{q^{m-1}}$ de $\mathbb{F}_{q^{m}}$.

Corolário 1.1.10. Seja $f(X) \in \mathbb{F}_{q}[X]$ um polinômio irredutivel de grau $m$. Então o corpo de decomposição de $f$ sobre $\mathbb{F}_{q}$ é $\mathbb{F}_{q^{m}}$.

Definição 1.1.11. Sejam $\mathbb{K}$ uma extensão de $\mathbb{F}_{q^{\prime}}$ de grau s e $\xi \in \mathbb{K}$. Entĩo os elementos $\xi, \xi^{q}, \xi^{q^{2}}, \ldots, \xi^{q^{s-1}}$ dizem-se conjugados de $\xi$ sobre $\mathbb{F}_{q}$. 
Os conjugados de $\xi \in \mathbb{K}$ com respeito a $\mathbb{F}_{q}$ são distintos se, e somente se, o polinômio minimal de $\xi$ sobre $\mathbb{F}_{q}$ tem grau $s$. Caso contrário, o grau $r$ de seu polinômio minimal é un divisor próprio de $s$ e, então os conjugados de $\xi$ sobre $\mathbb{F}_{q}$ são os elementos distintos $\xi, \xi^{q}, \xi^{q^{2}}, \ldots, \xi^{q^{r-1}}$.

Corolário 1.1.12. Seja $\xi \in \mathbb{F}_{q^{m}}$. Então o polinômio minimal de $\xi$ sobre $\mathbb{F}_{q}$ é

$$
\operatorname{irr}\left(\xi, \mathbb{F}_{q}\right)=(X-\xi)\left(X-\xi^{q}\right) \cdots\left(X-\xi^{q^{s-1}}\right)
$$

onde $s$ é o menor inteiro positivo tal que $\xi^{q^{s}}=\xi$.

Definição 1.1.13. Seja $n$ um inteiro positivo. O corpo de decomposição de $X^{n}-1$ sobre um corpo $\mathbb{F}_{q}$ é dito o n-ésimo corpo ciclotômico sobre $\mathbb{F}_{q} e$ denotaremos por $\mathbb{F}^{(n)}$.

As raízes de $X^{n}-1 \mathrm{em} \mathbb{F}^{(n)}$ são ditas as raízes n-ésimas da unidade sobre $\mathbb{F}_{q}$ e o conjunto de todas as raízes é denotado por $\mathbb{E}^{(n)}$.

Teorema 1.1.14. Seja n um inteiro positivo e seja $\mathbb{F}$ um corpo de característica $p$. Se $p$ não divide $n$ então $\mathbb{E}^{(n)}$ é um subgrupo cíclico, de ordem $n$, do grupo multiplicativo de $\mathbb{F}^{(n)}$.

Definição 1.1.15. Seja $\mathbb{F}$ um corpo de característica $p$ e seja $n$ um inteiro positivo tal que $p$ não divide $n$. Então um gerador do grupo cíclico $\mathbb{E}^{(n)}$ é dito uma raiz n-ésima primitiva da unidade sobre $\mathbb{F}$.

Existem exatamente $\phi(n)$ diferentes raízes n-ésimas primitivas da unidade sobre $\mathbb{F}$, onde $\phi$ é a função de Euler.

Se $\xi$ é uma delas então todas raízes n-ésimas primitivas da unidade sobre $\mathbb{F}$ são da forma $\xi^{s}$, onde $1 \leq s \leq n$ e $\operatorname{mdc}(s, n)=1$.

Suponhamos que $1, \xi, \xi^{2}, \ldots, \xi^{n-1}$ sejam as raízes de $X^{n}-1$ sobre $\mathbb{F}$.

Para $i=0,1, \ldots, n-1$, os conjugados de $\xi^{i}$ são

$$
\xi^{i}, \xi^{i q}, \xi^{i q^{2}}, \ldots, \xi^{i q^{s-1}}
$$

onde $s$ é o menor inteiro positivo tal que $\xi^{i q^{s}}=\xi^{i}$.

Note que,

$$
\xi^{i q^{s}}=\xi^{i} \Leftrightarrow \xi^{i q^{s}-i}=1 \Leftrightarrow n \mid\left(i q^{s}-i\right) \Leftrightarrow i q^{s} \equiv i \quad(\bmod n) .
$$

Portanto, podemos usar a condição $i q^{s} \equiv i \quad(\bmod n)$ para cleterminarmos os conjugados de $\xi$. 
Assim, o polinômio minimal cla raiz $\xi^{i}$ (e, portanto, também o clas raízes $\left.\xi^{i q}, \ldots, \xi^{i q^{s-1}}\right)$ é o produto

$$
m_{\xi^{i}}(X)=\left(X-\xi^{i}\right)\left(X-\xi^{i q}\right)\left(X-\xi^{i q^{2}}\right) \cdots\left(X-\xi^{i q^{s-1}}\right)
$$

onde $s$ é o menor inteiro positivo tal que $i q^{s} \equiv i \quad(\bmod n)$.

Seja $i$ um inteiro tal que $0 \leq i<n$.

Definição 1.1.16. A i-ésima classe ciclotômica de q módulo n é

$$
\Omega_{i}=\left\{i, i q, i q^{2}, \ldots, i q^{r_{i}-1}\right\}
$$

onde cada $i q^{j}$ é reduzido módulo $n$ e $r_{i}$ é o menor inteiro positivo tal que $i q^{r_{i}} \equiv i \quad(\bmod n)$.

Proposição 1.1.17. Os conjuntos $\Omega_{i}$ possuem as seguintes propriedades:

(i) $\operatorname{Se} \Omega_{i} \cap \Omega_{j} \neq \emptyset$ então $\Omega_{i}=\Omega_{j}$.

(ii) A união de todos $\Omega_{i}$ é igual $\mathbb{Z}_{n}$.

Teorema 1.1.18. Se $\mathbb{F}_{q^{m}}$ é o corpo de decomposição de $X^{n}-1$ sobre $\mathbb{F}_{q}$ então $\left[\mathbb{F}_{q^{m}}: \mathbb{F}_{q}\right]=m$ onde $m$ é o menor inteiro positivo tal que $n \mid\left(q^{m}-1\right)$, isto é, $m$ é o menor inteiro positivo tal que $q^{m} \equiv 1 \quad(\bmod n)$. Em outras palavras, $m$ é a ordem da classe de $q \mathrm{em} \mathbb{Z}_{n}$, que denotaremos por $m=o_{n}(q)$.

Teorema 1.1.19. Sejam $\xi$ uma raiz de $X^{n}-1$ no menor corpo finito $\mathbb{F}$ de característica p que contém $\xi$ e $m_{\xi}(X)$ seu polinômio minimal. Sejam $\zeta$ um elemento primitivo em $\mathbb{F}$ e $\xi=\zeta^{i}$. Se u é o menor elemento na i-ésima classe ciclotômica de q módulo $n$ então

$$
m_{\xi}(X)=\prod_{k \in \Omega_{u}}\left(X-\zeta^{k}\right) .
$$

Vamos construir um exemplo para aplicarmos a teoria de corpos finitos desenvolvida até aqui.

Exemplo 1.1.20. Seja $\zeta$ uma raiz de $m(X)=X^{3}+X+1$ sobre $\mathbb{F}_{2}$ em alguma extensão de $\mathbb{F}_{2}$ e seja $\mathbb{E}=\mathbb{F}_{2}(\zeta)$.

Afirmamos que o polinômio $m(X)=X^{3}+X+1$ é irredutível sobre $\mathbb{F}_{2}$.

Para verificarmos isto, mostraremos que $m(X)$ não pode ser escrito como produto de fatores lineares. De fato, $m(0)=1$ e $m(1)=1$, logo o polinômio $m(X)$ não tem fatores lineares. Assim, $m(X)$ é irredutível sobre $\mathbb{F}_{2}$. 
Como $\left[\mathbb{E}: \mathbb{F}_{2}\right]=\operatorname{grau}(m(X))=3$ têm-se $\mathbb{F}_{2}(\zeta)=\mathbb{F}_{2^{3}}=\mathbb{F}_{8}$ e $\left\{1, \zeta, \zeta^{2}\right\}$ é uma base de $\mathbb{F}_{2}(\zeta)$ sobre $\mathbb{F}_{2}$, clonde $\mathbb{F}_{2}(\zeta)=\left\{\lambda_{0}+\lambda_{1} \zeta+\lambda_{2} \zeta^{2} \mid \lambda_{0}, \lambda_{1}, \lambda_{2} \in \mathbb{F}_{2}\right\}=\mathbb{F}_{8}$.

Vamos mostrar que $\zeta$ é um elemento primitivo de $\mathbb{F}_{8}$ calculando suas potências e levando em conta que $\zeta^{3}=1+\zeta$. Logo, $|\langle\zeta\rangle|=7$ e portanto $\zeta$ é, de fato, um elemento primitivo de $\mathbb{F}_{8}$.

Na tabela abaixo descrevemos estas potências em função da base dada.

\begin{tabular}{|c||c|}
\hline expoente de $\zeta$ & polinômio \\
\hline 0 & 1 \\
\hline 1 & $\zeta$ \\
\hline 2 & $\zeta^{2}$ \\
\hline 3 & $\zeta+1$ \\
\hline 4 & $\zeta^{2}+\zeta$ \\
\hline 5 & $\zeta^{2}+\zeta+1$ \\
\hline 6 & $\zeta^{2}+1$ \\
\hline
\end{tabular}

As classes ciclotômicas módulo 7 são:

$$
\Omega_{0}=\{0\} ; \quad \Omega_{1}=\{1,2,4\} ; \quad \Omega_{3}=\{3,6,5\} .
$$

Logo, $X^{7}-1$ tem três fatores irredutíveis. Utilizando a tabela construída acima, temos que os polinômios minimais, de acordo com o Teorema 1.1.19, são:

$$
\begin{array}{lll}
m_{0}(X)=X-1 \\
m_{\zeta}(X)=(X-\zeta)\left(X-\zeta^{2}\right)\left(X-\zeta^{4}\right)=X^{3}+X+1 \\
m_{\zeta^{3}}(X)=\left(X-\zeta^{3}\right)\left(X-\zeta^{6}\right)\left(X-\zeta^{5}\right)=X^{3}+X^{2}+1
\end{array}
$$

Assim, a fatoração de $X^{7}-1$ em polinômios irredutíveis sobre $\mathbb{F}_{2}$ é

$$
X^{7}-1=(X+1)\left(X^{3}+X+1\right)\left(X^{3}+X^{2}+1\right) .
$$

Note que $\zeta$ e $\zeta^{3}$ são ambas raízes primitivas 7-ésima da unidade, mas seus polinômios minimais são diferentes.

Às vezes, precisamos apenas de informações sobre o número de polinômios minimais que há na fatoração de $X^{n}-1$.

Exemplo 1.1.21. Sejam $n=9$ e $p=2$. Vamos calcular as $i$-ésimas classes ciclotômicas de 2 módulo 9:

$$
\begin{aligned}
& \Omega_{0}=\{0\} ; \\
& \Omega_{1}=\{1.2,4,8,7,5\} ; \\
& \Omega_{3}=\{3,6\} .
\end{aligned}
$$

Logo, o polinômio $X^{9}-1$ sobre $\mathbb{F}_{2}$ têm um fator de grau 6 , um fator de grau 2 , e um fator de grau um. Cada um destes é um polinômio irredutível sobre $\mathbb{F}_{2}$. 
Observação: Note que se $\mathbb{F}_{p^{m}}=\mathbb{F}_{p}(\xi)$ isto não implica necessariamente que $\xi$ é um elemento primitivo no sentido aqui utilizado. Por exemplo, consideremos $p=3$ e $\xi$ uma raiz primitiva 4-ésima da unidade. Então $\xi$ é raiz de $X^{2}+1=0$, que é irredutível sobre $\mathbb{F}_{3}$. Logo, $\left[\mathbb{F}_{3}(\xi): \mathbb{F}_{3}\right]=2 \mathrm{e}$ $\mathbb{F}_{3}(\xi)=\mathbb{F}_{3^{2}}=\mathbb{F}_{9}$.

Como $\langle\xi\rangle=\{1, \xi,-1,-\xi\}$ é claro que $\xi$ não é um elemento primitivo de $\mathbb{F}_{9}$. Por outro lado, se tomarmos $\zeta=1+\xi$ temos que

$$
\begin{aligned}
\zeta^{2} & =2 \xi \\
\zeta^{3} & =-2+2 \xi \\
\zeta^{4} & =2 \\
\zeta^{5} & =2+2 \xi \\
\zeta^{6} & =\xi \\
\zeta^{7} & =-1+\xi \\
\zeta^{8} & =1
\end{aligned}
$$

Logo, $|\langle\zeta\rangle|=8$ e $\zeta$ é um elemento primitivo de $\mathbb{F}_{9}$.

Em geral, pode ser muito difícil achar a fatoração do polinômio $X^{n}-1$ sobre $\mathbb{F}_{q}$.

\subsection{Anéis Semisimples}

Nesta seção enunciaremos três teoremas da teoria de anéis semisimples que usaremos ao longo de nosso trabalho.

Utilizaremos como bibliografia básica para esta seção [17, capítulo I], [18, capítulos II].

Definição 1.2.1. Um R-módulo $M$ diz-se semisimples se todo submódulo de $M$ é um somando direto.

Definição 1.2.2. Um anel $R$ diz-se semisimples se $o r$-módulo ${ }_{R} R$ é semisimples.

Teorema 1.2.3. Seja $R$ um anel. As seguintes afirmações são equivalentes:

(i) Todo R-módulo é semisimples.

(ii) $R$ é um anel semisimples.

(iii) $R$ é soma direta de um número finito de ideais minimais à esqueda. 
Teorema 1.2.4. Seja $R$ um anel. Então $R$ é semisimples se, e somente se, todo ideal à esquerda $L$ de $R$ é da forma $L=R e$, onde e $\in R$ é um idempotente.

Em termos de idempotentes, os fatos apresentados acima, implicam o seguinte:

Teorema 1.2.5. Seja $R=\oplus_{i=1}^{t} L_{i}$ uma decomposição de um anel semisimples em soma direta de ideais à esquerda minimais. Então, existe uma familia $\left\{e_{1}, \ldots, e_{t}\right\}$ de elementos de $R$ tais que:

(i) $e_{i} \neq 0$ é um elemento idempotente, $1 \leq i \leq t$;

(ii) se $i \neq j$ então $e_{i} e_{j}=0$;

(iii) $1=e_{1}+\ldots+e_{t}$;

(iv) $e_{i}$ não pode ser escrito na forma $e_{i}=e_{i}^{\prime}+e_{i}^{\prime \prime}$ onde $e_{i}^{\prime}, e_{i}^{\prime \prime}$ são idempotentes tais que $e_{i}^{\prime}, e_{i}^{\prime \prime} \neq 0$ e e $e_{i}^{\prime} e_{i}^{\prime \prime}=0,1 \leq i \leq t ;$

(v) $L_{i}=R e_{i}$.

Reciprocamente, dada uma familia de idempotentes $\left\{e_{1}, \ldots, e_{t}\right\}$ satisfazendo as condições de (i) a (iv) então os ideais à esquerda $L_{i}=R e_{i}$ são minimais e $R=\oplus_{i=1}^{t} L_{i}$.

Se $R$ é um anel comutativo então os ideais $L_{i}, 1 \leq i \leq t$, são os únicos ideais minimais de $R$ e todo outro ideal é soma direta de alguns destes.

\subsection{Anéis de Polinômios}

Nesta seção apresentaremos alguns resultados da teoria de anéis de polinômios que nos serão utéis nos próximos capítulos. $\mathbb{F}$.

Consideremos o anel de polinômios em $X$ com coeficientes em um corpo

Teorema 1.3.1. Todo ideal de $\mathbb{F}[X]$ é principal.

Corolário 1.3.2. Seja $\mathcal{I} \neq\{0\}$ um ideal de $\mathbb{F}[X]$. Então existe um único polinômio mònico $f$ em $\mathcal{I}$, de grau mínimo, tal que $\mathcal{I}=\langle f\rangle$. 
Seja $\mathbb{F}$ um corpo finito com $q$ elementos.

Definimos $\mathcal{R}_{n}$ como sendo o anel quociente

$$
\mathcal{R}_{n}=\frac{\mathbb{F}[X]}{\left\langle X^{n}-1\right\rangle}
$$

Daclo $f \in \mathbb{F}[X]$ denotaremos por

$$
\overline{f(X)}=\left\{f(X)+g(X)\left(X^{n}-1\right) \mid g(X) \in \mathbb{F}[X]\right\}
$$

a sua classe em $\mathcal{R}_{n}$. $\overline{1}, \frac{\text { Note }}{X}, \ldots, \frac{X^{n-1}}{X^{n-1}}$.

Proposição 1.3.3. Todo ideal de $\mathcal{R}_{n}=\frac{\mathbb{F}[X]}{\left\langle X^{n}-1\right\rangle}$ é da forma $\langle\bar{g}\rangle$, onde $g$ é um divisor de $X^{n}-1$.

Dado um ideal $\mathcal{I}$ de $\mathcal{R}_{n}$ existe um único divisor mônico $g$ de $X^{n}-1$ tal que $\mathcal{I}=\langle\bar{g}\rangle$.

Definição 1.3.4. O polinômio g acima chama-se o polinômio gerador de $\mathcal{I}$.

Teorema 1.3.5. Sejam $\mathcal{I}$ e $\mathcal{J}$ ideais em $\mathcal{R}_{n}$ com polinômios geradores $\bar{g}_{1} e$ $\bar{g}_{2}$ respectivamente. Então

(i) $\mathcal{I} \subset \mathcal{J}$ se e somente se $g_{2}$ divide $g_{1}$ em $\mathbb{F}[X]$.

(ii) $\mathcal{I} \cap \mathcal{J}$ é gerado por $\overline{m m c\left(g_{1}, g_{2}\right)}$.

(iii) $\mathcal{I}+\mathcal{J}$ é gerado por $\overline{m d c\left(g_{1}, g_{2}\right)}$.

(iv) $\mathcal{I} \mathcal{J}$ é gerado por $\overline{m d c\left(g_{1} g_{2}, X^{n}-1\right)}$.

Vamos supor que $m d c(n, q)=1$. Seja $X^{n}-1=f_{1}(X) f_{2}(X) \cdots f_{t}(X)$ a decomposição do polinômio $X^{n}-1$ como produto de fatores mônicos irreclutíveis em $\mathbb{F}[X]$. Como $m d c(n, q)=1$ o polinômio $X^{n}-1$ é separavél sobre F e temos que $f_{i}(X) \neq f_{j}(X)$ se $i \neq j$. Com esta notação temos os seguintes. 
Teorema 1.3.6. Seja $\mathcal{I}$ um ideal em $\mathcal{R}_{n}$ com polinômio gerador g e seja $h=\left(X^{n}-1\right) / g$. Então $g$ e $h$ são relativamente primos e existem polinômios $r$ e s tais que

$$
r g+s h=1 .
$$

Ainda, $e=$ rg e temos que $\mathcal{I}=\langle\bar{e}\rangle=\mathcal{R}_{n} \bar{e}$ e $\bar{e}^{2}=\bar{e}$, donde $\bar{x} \bar{e}=\bar{x}$, para todo $\bar{x} \in \mathcal{I}$; isto é, todo ideal $\mathcal{I}$ de $\mathcal{R}_{n}$ é gerado por um idempotente $\bar{e}$ que é a identidade de $\mathcal{I}$.

Teorema 1.3.7. Se $\bar{e} \in \mathcal{R}_{n}$ é tal que $\bar{e}^{2}=\bar{e}$ e $\mathcal{I}=\langle\bar{e}\rangle$ então o polinômio gerador de $\mathcal{I}$ é

$$
g=m d c\left(e, X^{n}-1\right) .
$$

Demonstração: Sabemos que $X^{n}-1=h g$ e $e=r g$.

Temos que

$$
m d c\left(e, X^{n}-1\right)=m d c(r g, h g)
$$

Como $r$ e $h$ são relativamente primos, segue que

$$
m d c\left(e, X^{n}-1\right)=g \text {. }
$$

Proposição 1.3.8. O ideal $\mathcal{N}_{i}=\left\langle\bar{f}_{i}\right\rangle$ é um ideal maximal de $\mathcal{R}_{n}, 1 \leq i \leq t$.

Demonstração: Seja $\mathcal{I}$ um ideal em $\mathcal{R}_{n}$ tal que $\mathcal{N}_{i} \subset \mathcal{I} \subset \mathcal{R}_{n}$ e seja $g$ o polinômio gerador de $\mathcal{I}$. Como $\mathcal{N}_{i} \subset \mathcal{I}$ tem-se que $g$ divide $f_{i}$ em $\mathbb{F}[X]$. Como $f_{i}$ é irredutível devemos ter $g=1$ ou $g=f_{i}$ o que implica $\mathcal{I}=\mathcal{R}_{n}$ ou $\mathcal{I}=\mathcal{N}_{i}$.

Proposição 1.3.9. O ideal $\mathcal{M}_{i}$ gerado pelo polinômio $\widehat{f}_{i}=\left(X^{n}-1\right) / f_{i}$ é um ideal minimal de $\mathcal{R}_{n}$, para $1 \leq i \leq t$.

Demonstração: Seja $\mathcal{J}$ um ideal tal que $0 \subset \mathcal{J} \subset \mathcal{M}_{i}$ e seja $g \circ$ polinômio gerador de $\mathcal{J}$. Como $\mathcal{J} \subset \mathcal{M}_{i}$ tem-se que $\widehat{f}_{i}$ divide $g$ em $\mathbb{I}[X]$, o que implica que $f_{j}$ divide $g$, para todo $j \neq i$. Temos que $g \mid\left(X^{n}-1\right)$ então

$$
g=f_{1}^{\delta_{1}} f_{2}^{\delta_{2}} \cdots f_{t}^{\delta_{t}}, \quad \text { onde } \delta_{j}=0 \text { ou } 1, \quad 1 \leq j \leq t
$$

Logo,

$$
g=f_{1} f_{2} \cdots f_{i}^{\delta_{i}} \cdots f_{t}, \quad \text { onde } \quad \delta_{i}=0 \text { ou } 1
$$


Assim, se $\delta_{i}=0$ então $g=\widehat{f}_{i}$; se $\delta_{i}=1$ então $g=X^{n}-1$. Logo, $\mathcal{J}=0$ ou $\mathcal{J}=\mathcal{M}_{i}$ e segue a tese.

Sejam $\mathcal{M}_{i}=\left\langle\overline{\widehat{f}}_{i}\right\rangle$ um ideal minimal de $\mathcal{R}_{n}$ e $h_{i}=\left(X^{n}-1\right) / \widehat{f}_{i}$ segue, do Teorema 1.3.6, que existem polinômios $r_{i}$ e $s_{i}$ tais que $r_{i} \widehat{f}_{i}+s_{i} f_{i}=1 \mathrm{e}$ $\bar{e}_{i}=\bar{r}_{i} \overline{\widehat{f}}_{i}$ é um idempotente primitivo em $\mathcal{R}_{n}, 1 \leq i \leq t$.

Teorema 1.3.10. Com a notação acima tem-se que

$$
\mathcal{R}_{n}=\mathcal{M}_{1} \oplus \mathcal{M}_{2} \oplus \cdots \oplus \mathcal{M}_{t}
$$

Demonstração: Como $m d c\left(\widehat{f}_{1}, \widehat{f}_{2}, \ldots, \widehat{f}_{t}\right)=1$ segue, do algoritmo de Euclides, que existem $r_{1}, r_{2}, \ldots, r_{t} \in \mathbb{F}[X]$ tais que

$$
1=r_{1} \widehat{f_{1}}+r_{2} \widehat{f_{2}}+\cdots+r_{t} \widehat{f_{t}}
$$

Logo, para todo elemento, não nulo, $u$ pertencente a $\mathbb{F}[X]$ tem-se que

$$
u=u r_{1} \widehat{f}_{1}+u r_{2} \widehat{f}_{2}+\cdots+u r_{t} \widehat{f}_{t}
$$

Tomando classes módulo $X^{n}-1$ temos

$$
\bar{u}=\overline{u r_{1}} \overline{\widehat{f}_{1}}+\overline{u r_{2}} \overline{\widehat{f}_{2}}+\cdots+\overline{u r_{t}} \overline{\widehat{f}_{t}} \in \mathcal{M}_{1}+\mathcal{M}_{2}+\cdots+\mathcal{M}_{t}
$$

Portanto, $\mathcal{R}_{n}$ é soma dos ideais minimais $\mathcal{M}_{i}, 1 \leq i \leq t$.

Seja $v \in \mathbb{F}[X]$ tal que $\bar{v} \in \mathcal{M}_{i} \cap\left(\sum_{i \neq j} \mathcal{M}_{j}\right)$.

Como $\bar{v} \in \mathcal{M}_{i}$ existe $v_{i} \in \mathbb{F}[X]$ tal que $\bar{v}=v_{i} \widehat{f_{i}}+\left\langle X^{n}-1\right\rangle$. Logo, tem-se que $v=v_{i} \widehat{f}_{i}+\varphi_{i}\left(X^{n}-1\right)$, para algum $\varphi_{i} \in \mathbb{F}[X]$. Isto implica que $f_{j} \mid v$, $\forall j \neq i$.

Por outro lado, $\bar{v} \in \mathcal{M}_{j}$ então $\bar{v}=v_{j} \widehat{f}_{j}+\left\langle X^{n}-1\right\rangle$, o que implica, da mesma forma, que $f_{i} \mid v, \forall i \neq j$.

Portanto, $\left(X^{n}-1\right) \mid v$, o que implica que $\bar{v}=0$.

Assim, $\mathcal{R}_{n}$ é soma direta de ideais minimais $\mathcal{M}_{i}, 1 \leq i \leq t$.

Corolário 1.3.11. $\mathcal{R}_{n}$ é semisimples. 
Corolário 1.3.12. Seja $t$ um inteiro positivo. Sejam $\left\{i_{1}, \ldots, i_{s}\right\},\left\{j_{1}, \ldots, j_{t-s}\right\}$ uma partição do anel $\mathbb{Z}_{t}$. Então o ideal gerado por

$$
f_{i_{1}}(X) \cdots f_{i_{s}}(X)=\frac{X^{n}-1}{f_{j_{1}}(X) \cdots f_{j_{t-s}}(X)}
$$

é

$$
\bigoplus_{k=1}^{t-s} \mathcal{M}_{j_{k}}
$$

e o idempotente gerador deste ideal é

$$
e_{j_{1}}(X)+\cdots+e_{j_{t-s}}(X)=1-\left(e_{i_{1}}(X)+\cdots e_{i_{s}}(X)\right)
$$

Proposição 1.3.13. Seja $n_{i}=\operatorname{grau}\left(f_{i}\right)$. Então temos que

$$
\mathcal{M}_{i} \cong \frac{\mathbb{F}[X]}{\left\langle f_{i}\right\rangle} \cong \mathbb{F}_{p^{n_{i}}}
$$

Demonstração: Do Teorema 1.3.6 temos que $\mathcal{M}_{i}$ é gerado pelo idempotente $\bar{e}_{i}=\widehat{r_{i} \widehat{f}_{i}}$.

Definimos uma aplicação $\Phi: \mathbb{F}[X] \longrightarrow \mathcal{M}_{i}$ por:

$$
u \longmapsto \bar{u} \overline{e_{i}}
$$

É fácil ver que $\Phi$ é um epimorfismo. Logo, do teorema do homomorfismo, temos que

$$
\frac{\mathbb{F}[X]}{\operatorname{Ker}(\Phi)} \cong \mathcal{M}_{i}
$$

onde $\operatorname{Ker}(\Phi)=\{q \in \mathbb{F}[X] \mid \Phi(q)=\overline{0}\}$.

Note que, $f_{i} \nmid e_{i}$ isto equivale a dizer que $\operatorname{mdc}\left(f_{i}, e_{i}\right)=1$.

Um elemento $v \in \mathbb{F}[X]$ pertence ao $\operatorname{Ker}(\Phi)$ se, e somente se, $\bar{v} \bar{e}_{i}=$ $\overline{0}$, o que equivale a dizer que $\left(X^{n}-1\right) \mid v e_{i}$, ou seja, $\left(X^{n}-1\right) \mid v r_{i} \widehat{f}_{i}$, ou equivalentemente que, $f_{i} \mid v r_{i}$ e isto só ocorre se. e somente se, $f_{i} \mid v$.

$$
\text { Portanto, } \mathcal{M}_{i} \cong \frac{\mathbb{F}[X]}{\left\langle f_{i}\right\rangle} \text {. }
$$




\subsection{Anéis de Grupos}

Sejam $R$ um anel com uniclade e $G$ um grupo finito.

Definimos $R G$ como o conjunto de todas as combinações lineares formais do tipo

$$
\alpha=\sum_{g \in G} \alpha(g) g, \quad \text { onde } \quad \alpha(g) \in R .
$$

Note que a definição implica que dois elementos $\alpha=\sum_{g \in G} \alpha(g) g$, $\gamma=\sum_{g \in G} \gamma(g) g \in R G$ são iguais se e somente se $\alpha(g)=\gamma(g), \forall g \in G$.

O conjunto $\operatorname{supp}(\alpha)=\{g \in G \mid \alpha(g) \neq 0\}$ diz-se o suporte do elemento $\alpha$.

Define-se a soma de elementos de $R G$ por

$$
\left(\sum_{g \in G} \alpha(g) g\right)+\left(\sum_{g \in G} \gamma(g) g\right)=\sum_{g \in G}(\alpha(g)+\gamma(g)) g .
$$

E dados dois elementos $\alpha=\sum_{g \in G} \alpha(g) g, \gamma=\sum_{g \in G} \gamma(g) g \in R G$ podemos clefinir seu produto por:

$$
\alpha \gamma=\sum_{g, h \in G} \alpha(g) \gamma(h) g h
$$

onde $\alpha(g) \gamma(h)$ indica o produto em $R$ e $g h$ o produto de elementos em $G$.

É fácil verificar que, com as operações definidas acima, $R G$ é um anel com unidade. A unidade de $R G$ é o elemento $1=\sum_{g \in G} \alpha(g) g$, onde $\alpha\left(1_{G}\right)=1 \mathrm{e}$ $\alpha(g)=0 \forall g \neq 1_{G}\left(1_{G}\right.$ é o elemento unidade do grupo $\left.G\right)$.

Definição 1.4.1. O anel $R G$ chama-se anel de grupo de $G$ sobre $R$.

Definimos um produto de elementos de $R G$ por elementos de $R$ da seguinte forma

$$
\lambda\left(\sum_{g \in G} \alpha(g) g\right)=\sum_{g \in G}(\lambda \alpha(g)) g
$$

onde $\lambda$ pertence a $R$.

É fácil verificar que $R G$ é um $R$-módulo. Mais ainda, se $R$ é comutativo, segue que $R G$ é uma álgebra sobre $R$. Por causa disso, chamamos $R G$ de álgebra de grupo de $G$ sobre $R$. 
A função $i: G \longrightarrow R G$ definida por $i(x)=\sum_{g \in G} a(g) g$, onde $a(x)=1$ e $a(g)=0$ se $g \neq x$, é um monomorfismo (de grupos multiplicativos) e podemos iclentificar $g$ com sua imagem $i(G)$. Com esta identificação temos que $G$ é una base de $R G$ sobre $R$.

Proposição 1.4.2. Sejam $R$ um anel comutativo e $G, H$ grupos. Então $R(G \times H) \cong(R G) H$ (o anel de grupo de $H$ sobre o anel $R G$ ).

Proposição 1.4.3. Sejam $\left\{R_{i}\right\}_{i \in I}$ uma familia de aneis e $R=\oplus_{i \in I} R_{i}$. Então para qualquer grupo $G$ temos $R G \cong \oplus_{i \in I} R_{i} G$.

Proposição 1.4.4. Sejam $G=\langle a\rangle$, um grupo cíclico de ordem $n$ e $\mathbb{F}$ um corpo finito. Todo ideal de $\mathbb{F} G$ é da forma $\mathbb{F} G f(a)$, onde $f(X)$ é um divisor de $X^{n}-1$.

Definição 1.4.5. A aplicação $\varepsilon: R G \longrightarrow R$ dada por

$$
\varepsilon\left(\sum_{g \in G} \alpha(g) g\right)=\sum_{g \in G} \alpha(g)
$$

é um homomorfismo de anéis chamado de função de aumento de $R G$ e seu núcleo, que denotaremos por $\Delta(G)$, chamamos de ideal de aumento de $R G$.

Seja $H$ um subgrupo normal em $G$ e seja $\omega: G \longrightarrow G / H$ o epimorfismo canônico. Podemos definir então um homomorfismo $\varepsilon_{H}: R G \longrightarrow R(G / H)$ dado por

$$
\varepsilon_{H}\left(\sum_{g \in G} \alpha(g) g\right)=\sum_{g \in G} \alpha(g) \omega(g) .
$$

Com as notações acima denotaremos $\operatorname{Ker}\left(\varepsilon_{H}\right)$ por $\triangle(G, H)$.

Teorema 1.4.6 (Maschke). Seja $G$ um grupo. Então, o anel de grupo $R G$ é semisimples se, e somente se, as seguintes condições são válidas:

(i) $R$ é um anel semisimples.

(ii) $G$ é finito.

(iii) $\left|G_{1}\right|$ é invertivel em $R$. 
Corolário 1.4.7. Sejam $G$ um grupo finito e $\mathbb{K}$ u.m corpo. Então $\mathbb{K} G$ é semisimples se e somente se car $(\mathbb{K}) \nmid|G|$.

Teorema 1.4.8 (Perlis-Walker). Seja $G$ um grupo abeliano finito, de ordem $n$, e seja $\mathbb{K}$ um corpo tal que $\operatorname{car}(\mathbb{K}) \nmid|G|$. Então

$$
\mathbb{K} G \simeq \oplus_{d \mid n} a_{d} \mathbb{K}\left(\zeta_{d}\right)
$$

onde $\zeta_{d}$ denota uma raiz primitiva da unidade de ordem $d$ e $a_{d}=\frac{n_{d}}{\left[\mathbb{K}\left(\zeta_{d}\right): \mathbb{K}\right]}$. Nesta fórmula, $n_{d}$ denota o número de elementos de ordem d em $G$.

Corolário 1.4.9. Seja G um grupo abeliano finito de ordem n. Então

$$
\mathbb{Q} G \simeq \oplus_{d \mid n} a_{d} \mathbb{Q}\left(\zeta_{d}\right)
$$

onde $\zeta_{d}$ denota uma raiz primitiva da unidade de ordem d e $a_{d}$ é o número de subgrupos cíclicos (ou fatores cíclicos) de $G$.

Proposição 1.4.10. Sejam $G$ um grupo finito e $\mathbb{K}$ um corpo algebricamente fechado tal que car $(\mathbb{K}) \nmid|G|$. Então o número de componentes simples de $\mathbb{K} G$ é igual ao número de classes de conjugação de $G$.

Definição 1.4.11. Sejam $R G$ um anel de grupo e $X$ um subconjunto do grupo $G$, denotaremos por $\widehat{X}$ o seguinte elemento de $R G$

$$
\widehat{X}=\sum_{x \in X} x .
$$

Relembrando, se $e$ é um idempotente central no anel $R$ então ele induz uma decomposição de $R$ como soma direta de ideais bilaterais; $R=R e \oplus$ $R(1-e)$.

Lema 1.4.12. Sejam $R$ um anel com unidade e $H$ um subgrupo de um grupo $G$. Se $|H|$ é invertível em $R$ então $\widetilde{H}=\frac{1}{|H|} \widehat{H}$ é um idempotente de $R G$. Além disso. se $H \triangleleft G$ então $\widetilde{H}$ é central.

Proposição 1.4.13. Sejam $R$ um anel com unidade e $H$ um subgrupo normal do grupo $G$. Se $|H|$ é invertível em $R$ então

$$
R G=R G \widetilde{H} \oplus R G(1-\widetilde{H})
$$

como soma direta de anéis $e$

$$
R G \tilde{H} \simeq R(G / H) \quad \text { e } \quad R G(1-\tilde{H})=\Delta(G, H) .
$$




\subsection{Representações de Grupos}

Nesta seção daremos uma noção básica da teoria de representações. Para maiores detalhes consultar [18, capítulo IV].

Sejam $G$ um grupo, $\mathbb{K}$ um corpo e $V$ um $\mathbb{K}$-módulo livre de posto finito.

Definição 1.5.1. Uma representação de $G$ sobre $\mathbb{K}$, com espaço de representação $V$, é um homomorfismo de grupos $T: G \longrightarrow G L(V)$, onde $G L(V)$ denota o grupo dos $\mathbb{K}$-automorfismos de $V$. O posto de $V$ sobre $\mathbb{K}$ diz-se $o$ grau da representação $T$.

Para cada elemento $x \in G$ denotaremos por $T_{x}: V \longrightarrow V$ o $\mathbb{K}$-automorfismo correspondente. Portanto, se $a, b \in G$ tem-se que $T_{a b}=T_{a} \circ T_{b}$ e $T_{1}=I$.

Se fixarmos uma $\mathbb{K}$-base de $V$ podemos definir um isomorfismo $\tau$ entre $G L(V)$ e o grupo $G L(n, \mathbb{K})$ das matrizes invertível de ordem $n \times n$ sobre $\mathbb{K}$, associando a cada automorfismo $T \in G L(V)$ a sua matriz na base dada.

Definição 1.5.2. Uma representação matricial de $G$ sobre $\mathbb{K}$ de grau $n$ é um homomorfismo $T: G \longrightarrow G L(n, \mathbb{K})$.

Definição 1.5.3. Duas representações $T: G \longrightarrow G L(V), \bar{T}: G \longrightarrow G L(W)$ sobre um mesmo corpo $\mathbb{K}$ dizem-se equivalentes se existe um isomorfismo de espaços vetoriais $\tau: V \longrightarrow W$ tal que $\bar{T}_{x}=\tau \circ T_{x} \tau^{-1}$, para todo $x \in G$.

Definição 1.5.4. Duas representações matriciais $A: G \longrightarrow G L(n, \mathbb{K}), B$ : $G \longrightarrow G L(n, \mathbb{K})$ de um grupo $G$ sobre $\mathbb{K}$ dizem-se equivalentes se existe uma matriz invertivel $U \in G L(n, \mathbb{K})$ tal que $A(x)=U B(x) U^{-1}$, para todo $x \in G$.

Definição 1.5.5. Seja $T: G \longrightarrow G L(V)$ uma representação de $G$ sobre $\mathbb{K}$. Um subespaço $S$ de $V$ diz-se $G$-invariante sob $T$ se $T_{x}(S) \subset S$, para todo $x \in G$.

Uma representação $T: G \longrightarrow G L(V)$ diz-se irredutivel se os únicos subespaços de $V$ que são $G$-invariantes sob $T$ são $\{0\}$ e $V$. Caso contrário a representação diz-se redutível.

Teorema 1.5.6. O número de representações irredutíveis, não equivalentes, de um grupo $G$ é igual ao número de classes de conjugação de $G$. 


\subsection{Caracteres de Grupos}

Nesta seção definiremos caracteres de grupos e enunciaremos proposições e teoremas que nos serão úteis no capítulo V. Para maiores detalhes sugerimos [18, capítulo V].

Definição 1.6.1. Sejam $G$ um grupo e $V$ um espaço vetorial de dimensão finita sobre um corpo $\mathbb{K}$. Seja $T: G \longrightarrow G L(V)$ uma representação de $G$ sobre $\mathbb{K}$. A função $\chi: G \longrightarrow \mathbb{K}$ definida por

$$
\chi(x)=\operatorname{tr} T(x), \quad \forall x \in G
$$

diz-se o caracter de $G$ sobre $\mathbb{K}$ da representação $T$ (onde $\operatorname{tr} T(x)$ denota $o$ traço da matriz $T(x))$.

Se a representação $T$ é irredutível então $\chi$ diz-se um caracter irredutivel.

O caracter $\chi$ de $G$ tal que $\chi(g)=1$, para todo $g \in G$, chama-se caracter principal de $G$.

Sejam $G$ um grupo finito e $\mathbb{K}$ um corpo tal que $\operatorname{car}(\mathbb{K}) \nmid|G|$. Se $\chi_{1}, \chi_{2}, \ldots, \chi_{r}$ denotam os caracteres de um conjunto completo de representações irredutíveis, não equivalentes, de $G$ sobre $\mathbb{K}$. Temos os seguintes resultados:

Proposição 1.6.2. O conjunto de todos os caracteres de $G$ sobre $\mathbb{K}$ é o conjunto de combinações lineares, não nulas, da forma $\chi=\sum_{i=1}^{r} n_{i} \chi_{i}$, onde cada $n_{i}$ é um inteiro positivo, $1 \leq i \leq r$.

Teorema 1.6.3. Os idempotentes centrais primitivos de $\mathbb{K} G$ são da seguinte forma

$$
e_{i}=\frac{1}{|G|} \sum_{x \in G} \chi_{i}(1) \chi_{i}\left(x^{-1}\right) x .
$$

O próximo resultado é bem conhecido mas é difícil achar uma prova explícita em livros; por causa disso incluimos uma demonstração detalhada do mesmo.

Seja $G$ um grupo abeliano de ordem $n$, escrito multiplicativamente, com fatoração única (a menos da ordem e isomorfismo), em grupos cíclicos:

$$
G=\mathcal{C}_{p_{1}^{s_{1}}} \times \mathcal{C}_{p_{2}^{s_{2}}} \times \cdots \times \mathcal{C}_{p_{t}^{s_{t}}}
$$


onde os $p_{i}$ são primos, não necessariamente distintos, e os $\mathcal{C}_{p_{i}^{s_{i}}}=\left\langle a_{i}\right\rangle$ são grupos cíclicos de ordem $p_{i}^{s_{i}}$ gerados pelo elemento $a_{i}$.

Seja $K$ um corpo finito que contém as raízes $n$-ésimas da unidade e cuja característica não divide $n$. Então $K$ é o corpo de decomposição de $G$ e, como um grupo abeliano de ordem $n$ têm $n$ classes de conjugação, ele tem $n$ representações irredutíveis. Neste caso, não há diferença entre uma representação irredutível e seu caracter.

Definimos a multiplicação de caracteres por:

$$
\left(\chi_{i} \chi_{j}\right)(g)=\chi_{i}(g) \chi_{j}(g), \quad \forall g \in G .
$$

Os caracteres irredutíveis de um grupo abeliano formam um grupo com a multiplicação, denotaremos tal grupo por $G^{*}$ e, a identidade deste grupo é o caracter principal.

Teorema 1.6.4. Seja $G=\mathcal{C}_{p_{1}^{s_{1}}} \times \mathcal{C}_{p_{2}^{s_{2}}} \times \cdots \times \mathcal{C}_{p_{t}^{s_{t}}}$ um grupo abeliano finito $e$ seja $K$ um corpo que contém todas as raízes $|G|$-ésimas da unidade. Então

(i) Os grupos $G, G^{*}$ e $G^{* *}$ são isomorfos.

(ii) Se $H$ é um subgrupo de $G$ então todo caracter $\varphi: G / H \longrightarrow K$ induz um caracter de $G$ via composição $G \stackrel{\omega}{\longrightarrow} G / H \stackrel{\varphi}{\longrightarrow} K e$

$$
\left(\frac{G}{H}\right)^{*} \cong H^{o}=\{\chi \in G \mid \chi(g)=1, \quad \forall g \in H\} .
$$

Demonstração: ( $i$ ) Para cada índice $i, 1 \leq i \leq t$, consideremos uma raiz $p_{i}^{s_{i}}$-ésima primitiva da unidade e definimos um caracter $\chi_{i}: G \longrightarrow K$, nos elementos $\left\{a_{i}\right\}_{1 \leq i \leq t}$ por

$$
\chi_{i}\left(a_{j}\right)=\left\{\begin{array}{lll}
\zeta_{i} & \text { se } & i=j \\
1 & \text { se } & i \neq j
\end{array}\right.
$$

e, num elemento $g \in G$ arbitrário, estendemos multiplicativamente: se $g=$ $a_{1}^{l_{1}} a_{2}^{l_{2}} \cdots a_{t}^{l_{t}}$ então definimos

$$
\chi_{i}(g)=\chi_{i}\left(a_{i}^{l_{i}}\right)
$$

Seja $\chi$ um elemento arbitrário de $G^{*}$ e seja $\alpha_{i}=\chi\left(a_{i}\right), 1 \leq i \leq t$. Então, como $a_{i}$ tem ordem $p_{i}^{s_{i}}$ temos

$$
\chi\left(a_{i}\right)^{p_{i}^{s_{i}}}=\chi\left(a_{i}^{p_{i}^{s_{2}}}\right)=\chi(1)=1=\alpha_{i}^{p_{i}^{s_{i}}}
$$


que implica que $\alpha_{i}$ é uma raiz $p_{i}^{s_{i}}$-ésima da uniclade; logo uma potência de $\zeta_{i}$.

Portanto, podemos escrever

$$
\chi\left(a_{i}\right)=\zeta_{i}^{k_{i}}
$$

para algum inteiro positivo $k_{i}$.

Cada elemento $g \in G$ pode-se escrever na forma $g=a_{1}^{l_{1}} a_{2}^{l_{2}} \cdots a_{t}^{l_{t}}$, com $0 \leq l_{i}<p_{i}^{s_{i}}$. Logo,

$$
\begin{aligned}
\chi(g) & =\chi\left(a_{1}^{l_{1}} a_{2}^{l_{2}} \cdots a_{t}^{l_{t}}\right) \\
& =\chi\left(a_{1}^{l_{1}}\right) \chi\left(a_{2}^{l_{2}}\right) \cdots \chi\left(a_{t}^{l_{t}}\right) \\
& =\prod_{i=1}^{t} \chi\left(a_{i}^{l_{i}}\right) \\
& =\prod_{i=1}^{t} \zeta_{i}^{l_{i} k_{i}} \\
& =\prod_{i=1}^{t} \chi_{i}\left(a_{i}^{l_{i}}\right)^{k_{i}} \\
& =\prod_{i=1}^{t} \chi_{i}^{k_{i}}(g) .
\end{aligned}
$$

Assim, concluímos que um caracter arbitrário de $G^{*}$ pode ser expresso como produto da "base" de caracteres $\chi_{i}$, para $i=1, \ldots, t$.

Vamos definir uma aplicação $f: G \longrightarrow G^{*}$ por

$$
g=a_{1}^{l_{1}} a_{2}^{l_{2}} \cdots a_{t}^{l_{t}} \stackrel{f}{\longmapsto} \chi_{g}=\chi_{1}^{l_{1}} \chi_{2}^{l_{2}} \cdots \chi_{t}^{l_{t}} .
$$

Claramente $f$ é um homomorfismo de grupos.

Note que a observação acima mostra que $f$ é sobrejetora. Vamos mostrar que $f$ também é injetora. Se $g \in \operatorname{Ker}(f)$ então $f(g)=\chi_{g}=1$, isto é, $\chi_{1}^{l_{1}} \chi_{2}^{l_{2}} \cdots \chi_{t}^{l_{t}}=1$. Então para todo $a_{i} \in G$ temos que

$$
\begin{aligned}
1 & =\chi_{1}^{l_{1}} \chi_{2}^{l_{2}} \cdots \chi_{t}^{l_{t}}\left(a_{i}\right) \\
& =\chi_{i}\left(a_{i}^{l_{i}}\right) \\
& =\zeta_{i}^{l_{i}} .
\end{aligned}
$$

com $l_{i}<p_{i}^{s_{i}}$ tem-se que $l_{i}=0,1 \leq i \leq t$, on seja, $g=1$. 
Portanto, $f$ é um isomorfismo de $G$ sobre $G^{*}$.

Observamos que $G^{*}$ é um grupo abeliano e $G^{* *}$ é seu dual, logo da demonstração acima, segue que $G^{*} \cong G^{* *}$ e, consequentemente, também $G \cong G^{* *}$.

(ii) Sejam $\omega: G \longrightarrow G / H$ o epimorfismo canônico e, $\varphi: G / H \longrightarrow K$ um caracter clo quociente no corpo $K$.

Claramente, a função $\chi=\varphi \circ \omega$ é um homomorfismo de $G$ em $K$, isto é, um caracter de $G$.

Agora, considere a aplicação $\Psi:(G / H)^{*} \longrightarrow H^{o}$ clefinida por

$$
\varphi \longmapsto \Psi(\varphi)=\varphi \circ \omega \text {. }
$$

Novamente, é claro que a aplicação $\Psi$ é um homomorfismo de grupos.

Vamos mostrar que $\Psi$ é injetora. Se $\varphi \in \operatorname{Ker} \Psi$ então $\chi=\varphi \circ \omega$ é o caracter principal. Logo, $1=\chi(G)=\varphi \circ \omega(G)=\varphi(G / H)$. Isto implica que $\varphi$ é o caracter principal $G / H$.

Vamos mostrar que $\Psi$ é sobrejetora. Seja $\chi \in H^{o}$ arbitrário. Definimos uma aplicação $\varphi: G / H \longrightarrow K$ por

$$
\bar{g} \longmapsto \varphi(\bar{g})=\chi(g) .
$$

Vamos mostrar que $\varphi$ está bem definida. De fato, sejam $g_{1}, g_{2} \in G$ tais que $\bar{g}_{1}=\bar{g}_{2}$. Então, $g_{1}=g_{2} h$, para algum $h \in H$. Logo, $\chi\left(g_{1}\right)=\chi\left(g_{2} h\right)=$ $\chi\left(g_{2}\right) \chi(h)=\chi\left(g_{2}\right)$. Portanto, $\varphi$ está bem definida.

Agora, temos

$$
\Psi(\varphi)(g)=\varphi \circ \omega(g)=\varphi(\bar{g})=\chi(g), \quad \forall g \in G
$$

isto é, $\Psi(\varphi)=\chi$, donde $\Psi$ é sobrejetora.

Portanto, $\Psi$ é isomorfismo.

A demonstração cla existência dos isomorfismos do item $(i)$ acima depende da escolha de uma decomposição para $G$ e de geradores para os grupos cíclicos correspondentes. Porém, é possível estabelecer um isomorfismo canônico $G \cong G^{* *}$, como mostraremos a seguir.

Para cada $g \in G$ definimos o caracter $\phi_{g} \in G^{* *}$ da seguinte maneira:

$$
\chi \in G^{*} \stackrel{\phi_{g}}{\longmapsto} \phi_{g}(\chi)=\chi(g) \in K
$$


Considere a aplicação $\Phi: G \longrightarrow G^{* *}$ dada por

$$
g \longmapsto \Phi(g)=\phi_{g}
$$

Esta aplicação $\Phi$ é um homomorfismo de grupos, isto é,

$$
\Phi(x y)=\Phi(x) \Phi(y) \quad \forall x, y \in G .
$$

Vamos mostrar que $\Phi$ é injetora. Se $g \in \operatorname{Ker}(\Phi)$ então $\Phi(g)=1$. Seja $g=a_{1}^{l_{1}} a_{2}^{l_{2}} \cdots a_{t}^{l_{t}}$ a decomposição de $g$. Para cada índice $i, 1 \leq i \leq t$, temos

$$
1=\phi_{g}\left(\chi_{i}\right)=\chi_{i}(g)=\chi_{i}\left(a_{i}^{l_{i}}\right)=\zeta_{i}^{l_{i}} .
$$

$\operatorname{logo} l_{i}=0,1 \leq i \leq t$, o que implica que $g=1$.

Dado $\chi \in G^{*}$ já mostramos que existe $g \in G$ tal que $\chi=\chi_{g}=\Phi(g)$. Logo, $\Phi$ é sobrejetora.

Portanto, $\Phi$ é um isomorfismo.

Proposição 1.6.5. Com as notações acima temos:

$$
\left(H^{\circ}\right)^{\circ}=\Phi(H) \text {. }
$$

Demonstração: Vamos mostrar inicialmente que $\Phi(H) \subset\left(H^{\circ}\right)^{\circ}$.

Seja $\phi_{h} \in \Phi(H)$ um elemento qualquer e, seja $\chi \in H^{\circ}$. Tem-se que

$$
\phi_{h}(\chi)=\chi(h)=1
$$

ou seja, $\phi_{h} \in\left(H^{o}\right)^{o}$.

Da parte $(i i)$ do Teorema 1.6.4 sabemos que $\left(H^{o}\right)^{o} \cong\left(G^{*} / H^{\circ}\right)^{*} \operatorname{logo}$

$$
\left|\left(H^{o}\right)^{o}\right|=\left|\left(\frac{G^{*}}{H^{o}}\right)^{*}\right|=\left|\frac{G^{*}}{H^{o}}\right|=\frac{|G|}{\left|H^{o}\right|} .
$$

Como $H^{o} \cong(G / H)^{*}$ tem-se que

$$
\left|\left(H^{o}\right)^{o}\right|=\frac{|G|}{\left|(G / H)^{*}\right|}=\frac{|G|}{|G| /|H|}=|H|=|\Phi(H)| .
$$

Como $\Phi(H) \subset\left(H^{\circ}\right)^{o}$ segue que $\left(H^{\circ}\right)^{o}=\Phi(H)$.

Como a função $f: G \longrightarrow G^{*}$ definida na demonstração do Teorema 1.6.4 é $u$ isomorfismo de grupos, ele define, de maneira natural, uma bijeção 
entre o conjunto de subgrupos de $G$ e o conjunto de subgrupos de $G^{*}$ que denotaremos ainda por $f$ e denotaremos também a inversa desta bijeção por $f^{-1}$.

Vamos definir agora uma bijeção $\eta$ entre o conjunto de subgrupos de $G$ e o conjunto de subgrupos de $G^{*}$. Para cada subgrupo $H$ de $G$ definimos $\eta(H)=H^{\circ}$.

Definiremos também uma aplicação $\eta^{\prime}$ entre o conjunto de subgrupos de $G^{*}$ e o conjunto de subgrupos de $G^{* *}$ da seguinte maneira: para cada subgrupo $S$ de $G^{*}$ definimos $\eta^{\prime}(S)=(S)^{\circ}$.

Em particular, para um subgrupo $H$ de $G$ temos $\eta^{\prime} \circ \eta(H)=\Phi(H)$.

Como $\Phi$ induz uma bijeção entre o conjunto de subgrupos de $G$ e o conjunto de subgrupos de $G^{* *}$ segue que $\eta^{\prime} \circ \eta$ é uma bijeção, o que implica que, $\eta$ é injetora.

Observe que o número de subgrupos de $G$ é igual ao número de subgrupos de $G^{*}$, pois $G$ e $G^{*}$ são isomorfos. Como $\eta$ é injetora segue que também é sobrejetora. Portanto, é uma bijeção.

Temos então as seguintes bijeções.

$$
\left\{\begin{array}{c}
\text { subgrupos } \\
\text { de } \\
G
\end{array}\right\} \stackrel{\eta}{\longrightarrow}\left\{\begin{array}{c}
\text { subgrupos } \\
\text { de } \\
G^{*}
\end{array}\right\} \stackrel{f^{-1}}{\longrightarrow}\left\{\begin{array}{c}
\text { subgrupos } \\
d e \\
G
\end{array}\right\}
$$

Sejam $\mathcal{C}$ a família de todos os subgrupos cíclicos de $G$ e $\mathcal{S}$ o conjunto de todos os subgrupos $H$ de $G$ tal que o quociente $G / H$ é cíclico.

Teorema 1.6.6. Com as notações acima temos:

$$
f^{-1} \circ \eta(\mathcal{S})=\mathcal{C}
$$

Demonstração: Seja $H$ um subgrupo de $G$ tal que $G / H$ é cíclico, isto é, um elemento de $\mathcal{S}$. Então $(G / H)^{*}$ também é cíclico e, como $(G / H)^{*} \cong H^{o}$, segue que $H^{\circ}$ é cíclico. Assim, $\eta(H)=H^{o}$ é cíclico. Logo, $f^{-1}(\eta(H))=$ $f^{-1}\left(H^{o}\right)$ é também cíclico, pois $f^{-1}$ é um isomorfismo, donde $f^{-1} \circ \eta(H) \in \mathcal{C}$. Consequentemente, $f^{-1} \circ \eta(\mathcal{S}) \subset \mathcal{C}$.

Note que todas as implicações acima podem ser revertidas de modo que, de forma analoga. pode-se provar que $\mathcal{C} \subset f^{-1} \circ \eta(\mathcal{S})$, clonde segue a ignaldade. 
Observamos que, da demonstração acima, segue que o número de subgrupos cíclicos de $G$ é igual ao número de subgrupos $H$ de $G$ tais que $G / H$ é cíclico e que subgrupos correspondentes na bijeção estabelecida tem a mesma ordem.

\subsection{Idempotentes}

Sejam $G=\left\{1, a, \ldots, a^{n-1}\right\}, a^{n}=1$, um grupo cíclico de ordem $n$ e $\mathbb{F}$ um corpo finito com $q$ elementos tal que $\operatorname{car}(\mathbb{F}) \nmid|G|$.

Vamos determinar os elementos idempotentes de $\mathbb{F} G$.

Considere a aplicação $\Theta: \mathbb{F}[X] /\left\langle X^{n}-1\right\rangle \longrightarrow \mathbb{F} G$ definida por:

$$
\bar{g} \mapsto g(a)
$$

É fácil ver que $\Theta$ é um isomorfismo de anéis.

Note que neste isomorfismo o elemento $a \in G$ corresponde à classe $X+$ $\left\langle X^{n}-1\right\rangle \in \mathcal{R}_{n}$.

Segue do Teorema 1.3.10 que

$$
\frac{\mathbb{F}[X]}{\left\langle X^{n}-1\right\rangle}=\mathcal{R}_{n} e_{1} \oplus \cdots \oplus \mathcal{R}_{n} e_{t}
$$

Portanto,

$$
\mathbb{F} G=\Theta\left(\mathcal{R}_{n}\right)=\mathbb{F} G \Theta\left(e_{1}\right) \oplus \cdots \oplus \mathbb{F} G \Theta\left(e_{t}\right) .
$$

Note que, $\overline{e_{i}(X)}=r_{i}(\bar{X}) \widehat{f}_{i}(\bar{X})$ donde $\Theta\left(\overline{e_{i}(X)}\right)=r_{i}(a) \widehat{f}_{i}(a)$.

Mostramos assim que os iclempotentes de $\mathbb{F} G$ são da forma

$$
\varepsilon_{i}=r_{i}(a) \widehat{f}_{i}(a), 1 \leq i \leq t .
$$

Exemplo 1.7.1. Sejam $\mathbb{F}$ o corpo finito com dois elementos e $G$ um grupo cíclico de ordem 7. Segue do Exemplo 1.1.20 que a decomposição de $X^{7}-1$ em fatores irredutíveis de $\mathbb{F}[X]$ é

$$
X^{7}-1=(X-1)\left(X^{3}+X^{2}+1\right)\left(X^{3}+X+1\right) .
$$

Do Teorema 1.3.10 temos

$$
\frac{\mathbb{F}[X]}{\left\langle X^{7}-1\right\rangle}=\mathcal{M}_{1} \oplus \mathcal{M}_{2} \oplus \mathcal{M}_{3}
$$

oncle cada $\mathcal{M}_{i}$ é um ideal minimal em $\mathbb{F}[X] /\left\langle X^{7}-1\right\rangle, i=1,2,3$.

Temos que 
- $\mathcal{M}_{1}$ é gerado pelo polinômio $g_{0}=X^{6}+X^{5}+X^{4}+X^{3}+X^{2}+X+1$;

- $\mathcal{M}_{2}$ é gerado pelo polinômio $g_{1}=X^{4}+X^{3}+X^{2}+1$;

- $\mathcal{M}_{3}$ é gerado pelo polinômio $g_{2}=X^{4}+X^{2}+X+1$;

Vamos aplicar o Teorema 1.3.6 em cada fator da decomposição acima.

Como $g_{0}=X^{6}+X^{5}+X^{4}+X^{3}+X^{2}+X+1$, temos que $h_{0}=X+1$ e, segue facilmente que

$$
g_{0}+\left(X^{5}+X^{3}+X\right) h_{0}=1 .
$$

Portanto, $e_{0}=g_{0}$.

Como $g_{1}=X^{4}+X^{3}+X^{2}+1$, temos que $h_{1}=X^{3}+X^{2}+1$ e, é fácil ver que

$$
\left(1+X^{2}\right) g_{1}+X^{3} h_{1}=1 \text {. }
$$

Portanto, $e_{1}=\left(1+X^{2}\right) g_{1}=X^{6}+X^{5}+X^{3}+1$.

Como $g_{2}=X^{4}+X^{2}+X+1$, temos que $h_{2}=X^{3}+X+1$ e, segue facilmente que

$$
g_{2}+X h_{2}=1
$$

Portanto, $e_{2}=g_{2}$.

Assim, os idempotentes de $\mathbb{F} G$ são:

$$
\begin{aligned}
& \varepsilon_{0}=a^{6}+a^{5}+a^{4}+a^{3}+a^{2}+a+1 \\
& \varepsilon_{1}=a^{6}+a^{5}+a^{3}+1 \\
& \varepsilon_{2}=a^{4}+a^{2}+a+1
\end{aligned}
$$

Exemplo 1.7.2. Sejam $\mathbb{F}$ o corpo finito com três elementos e $G$ um grupo cíclico de ordem 7. Vamos encontrar a decomposição de $X^{7}-1$ em fatores irredutíveis sobre $\mathbb{F}$.

As classes ciclotômicas de 3 módulo 7 são:

$$
\begin{aligned}
& \Omega_{0}=\{0\} ; \\
& \Omega_{1}=\{1,3,2,6,4,5\} .
\end{aligned}
$$

Assim, a fatoração de $X^{7}-1$ em fatores irredutíveis sobre $\mathbb{F}$ é:

$$
X^{7}-1=(X+2)\left(X^{6}+X^{5}+X^{4}+X^{3}+X^{2}+X+1\right) .
$$

Do Teorema 1.3.10 temos

$$
\frac{\mathbb{F}[X]}{\left\langle X^{\top}-1\right\rangle}=M_{1} \oplus M_{2}
$$


onde $\mathcal{M}_{1}=\langle X-1\rangle$ e $\mathcal{M}_{2}=\left\langle X^{6}+X^{5}+X^{4}+X^{3}+X^{2}+X+1\right\rangle$ são os ideais minimais em $\mathbb{F}[X] /\left\langle X^{7}-1\right\rangle$.

Vamos aplicar o Teorema 1.3.6 ein cacla fator $\mathcal{M}_{i}$ acima.

Seja $g_{0}=X+2$, temos que $h_{0}=X^{6}+X^{5}+X^{4}+X^{3}+X^{2}+X+1$ e, segue facilmente que

$$
\left(2 X^{5}+X^{4}+2 X^{2}+X\right) g_{0}+h_{0}=1
$$

Portanto,

$$
\begin{aligned}
e_{0} & =\left(2 X^{5}+X^{4}+2 X^{2}+X\right) g_{0} \\
& =2 X^{6}+2 X^{5}+2 X^{4}+2 X^{3}+2 X^{2}+2 X
\end{aligned}
$$

E é fácil ver que, $e_{1}=h_{0}=X^{6}+X^{5}+X^{4}+X^{3}+X^{2}+X+1$.

Assim, os idempotentes de $\mathbb{F} G$ são:

$$
\begin{aligned}
& \varepsilon_{0}=2 a^{6}+2 a^{5}+2 a^{4}+2 a^{3}+2 a^{2}+2 a \\
& \varepsilon_{1}=a^{6}+a^{5}+a^{4}+a^{3}+a^{2}+a+1 .
\end{aligned}
$$




\section{Capítulo 2}

\section{O Número de Componentes Simples}

\subsection{Introdução}

O cálculo do número de componentes simples da álgebra de grupo de um grupo finito pode ser obtido do Teorema de Berman-Witt (ver, [3, Teorema 21.5 e 21.25] ou [5, Teorema 47.2]) e do resultado de Khülshammer [12], usando a Teoria de caracteres. Ferraz em [6] estendeu este resultado para corpos de característica arbitrária que não divide a ordem de $G$.

Neste capítulo vamos expor com detalhes uma forma simplificada deste cálculo para o caso dos grupos abelianos que foi dada em [7]. Trata-se de um cálculo elementar que usa apenas a estrutura algébrica de $\mathbb{F} A$.

Sejam $\mathbb{F}$ um corpo finito com $|\mathbb{F}|=q$ elementos, $A$ um grupo abeliano finito tal que $m d c(q,|A|)=1$. Então $\mathbb{F} A$ é semisimples e, se $\left\{e_{1}, e_{2}, \ldots e_{r}\right\}$ é o conjunto de idempotentes primitivos de $\mathbb{F} A$ temos que

$$
\mathbb{F} A=\bigoplus_{i=1}^{r}(\mathbb{F} A) e_{i} \cong \bigoplus_{i=1}^{r} \mathbb{F}_{i},
$$

onde $\mathbb{F}_{i} \cong(\mathbb{F} A) e_{i}, 1 \leq i \leq r$, são corpos que são extensões finitas de $\mathbb{F}$.

Seja

$$
\mathcal{D}=\bigoplus_{i=1}^{r} \mathbb{F} e_{i} .
$$

Sote que $\mathbb{F} e_{i} \cong \mathbb{F}$ como corpos na forma natural e portanto o número 
$r$ de componentes simples é também a dimensão de $\mathcal{D}$ como espaço vetorial sobre $\mathbb{F}$.

Lema 2.1.1. Seja $\alpha$ um elemento de $\mathbb{F} A$. Então $\alpha \in \mathcal{D}$ se, e somente se, $\alpha^{q}=\alpha$.

Demonstração: Dado $\alpha \in \mathbb{F} A$, podemos escrever

$$
\alpha=\sum_{i=1}^{r} \alpha_{i}, \quad \text { com } \quad \alpha_{i}=\alpha e_{i} \in(\mathbb{F} A) e_{i}, 1 \leq i \leq r .
$$

Agora, $\alpha \in \mathcal{D}$ se e somente se cada $\alpha_{i} \in \mathbb{F} e_{i}$, para todo índice $i$.

Como $\mathbb{F} e_{i} \cong \mathbb{F}$, é o corpo de decomposição do polinômio $X^{q}-X$, tem-se que $\alpha_{i} \in \mathbb{F} e_{i}$ se e somente se $\alpha_{i}^{q}=\alpha_{i}$ para todo índice $i$.

Note que,

$$
\begin{aligned}
\alpha^{q} & =\left(\alpha_{1}+\alpha_{2}+\cdots+\alpha_{r}\right)^{q} \\
& =\alpha_{1}^{q}+\alpha_{2}^{q}+\cdots+\alpha_{r}^{q} .
\end{aligned}
$$

Portanto, $\alpha_{i}^{q}=\alpha_{i}$ para todo índice $i$, se e somente se, $\alpha^{q}=\alpha$.

Seja $h$ um elemento no grupo abeliano finito $A$. Definiremos a $q$-classe ciclotômica de $h$ em A como o conjunto

$$
\mathcal{S}_{h}=\left\{h^{q^{j}} \mid 0 \leq j \leq t_{h}-1\right\}
$$

onde $t_{h}$ é o menor inteiro positivo tal que

$$
q^{t_{h}} \equiv 1 \quad(\bmod o(h))
$$

onde $o(h)$ denota a ordem de $h$. Como $\operatorname{mdc}(q, o(h))=1$ existirá sempre tal número $t_{h}$.

Lema 2.1.2. Se $\mathcal{S}_{h} \neq \mathcal{S}_{k}$ então $\mathcal{S}_{h} \cap \mathcal{S}_{k}=\emptyset$.

Demonstração: Suponhamos que $x \in \mathcal{S}_{h} \cap \mathcal{S}_{k}$ então $x=h^{q^{j}}$, para algum inteiro $j$. Logo, é imediato que $\mathcal{S}_{x} \subset \mathcal{S}_{h}$. Como mdc $(q, o(h))=1$ segue que $o(x)=o(h)$.

Queremos encontrar o menor inteiro positivo $s$ tal que $\left(h^{q^{j}}\right)^{q^{s}}=h^{q^{j}}$. Assim,

$$
\left.h^{q^{j}\left(q^{s}-1\right)}=1 \Longleftrightarrow o(x) \mid\left(q^{s}-1\right) \Longleftrightarrow q^{s} \equiv 1 \quad \text { (mol } o(x)\right) .
$$


Como $o(x)=o(h)$ e $t_{h}$ é o menor inteiro positivo tal que

$$
q^{t_{h}} \equiv 1 \quad(\bmod o(h))
$$

segue que $s=t_{h}$. Portanto, $\mathcal{S}_{x}=\mathcal{S}_{h}$.

Da mesma forma, segue que $\mathcal{S}_{x}=\mathcal{S}_{k}$.

Portanto, $\mathcal{S}_{h}=\mathcal{S}_{k}$.

Lema 2.1.3. Seja $\alpha$ um elemento de D. Se $\alpha=\sum_{h \in A} \alpha_{h} h$ entäo $\alpha_{h}=$ $\alpha_{h^{q}}=\cdots=\alpha_{h^{q^{t^{-1}}}}$, para cada $h \in A$.

Demonstração: Seja $\alpha \in \mathbb{F} A$ tal que $\alpha \in \mathcal{D}$. Segue, do Lema 2.1.1, que $\alpha^{q}=\alpha$.

Portanto, se $\alpha=\sum_{h \in A} \alpha_{h} h$ temos que

$$
\alpha=\sum_{h \in A} \alpha_{h} h=\left(\sum_{h \in A} \alpha_{h} h\right)^{q}=\sum_{h \in A} \alpha_{h}^{q} h^{q} .
$$

Como $\alpha_{h} \in \mathbb{F}$ segue que $\alpha_{h}^{q}=\alpha_{h}$ e portanto

$$
\alpha=\sum_{h \in A} \alpha_{h} h=\sum_{h \in A} \alpha_{h} h^{q} .
$$

Como $h \in \operatorname{supp}(\alpha)$ então $h$ comparece em um dos termos do primeiro membro da igualdade acima. Logo, $h^{q} \in \operatorname{supp}(\alpha)$ e deve comparecer com o mesmo coeficiente nos dois membros da igualdade. Assim, $\alpha_{h^{q}}=\alpha_{h}$.

Repetindo essa construção obtemos, para cada $h \in A$,

$$
\alpha_{h}=\alpha_{h^{q}}=\cdots=\alpha_{h^{q} t^{t-1}} .
$$

\subsection{O número de componentes simples}

Seja $\mathcal{T}=\left\{h_{1}, h_{2}, \ldots, h_{r}\right\}$ um conjunto de representantes das $q$-classes ciclotômicas de $A$.

Teorema 2.2.1. Sejam $\mathbb{F} u m$ corpo finito com $|\mathbb{F}|=q$ elementos e. $A$ um grupo abeliano finito tal que $\operatorname{mdc}(q,|A|)=1$. Então o número de componentes simples de FA é igual ao número de q-classes ciclotômicas de. A. 
Demonstração: Como observado anteriormente, o número de componentes simples de $\mathbb{F} A$ é igual a dimensão de $\mathcal{D}$ sobre $\mathbb{F}$.

Iremos exibir uma base clesta subálgebra.

Dado uma $q$-classe ciclotômica $\mathcal{S}_{h}$ definiremos

$$
\eta_{h}=\sum_{x \in \mathcal{S}_{h}} x \in \mathbb{F} A
$$

Afirmamos que $\mathcal{B}=\left\{\eta_{h_{i}} \mid 1 \leq i \leq r\right\}$ é uma $\mathbb{F}$-base de $\mathcal{D}$.

Como os suportes de $\eta_{h_{1}}, \ldots, \eta_{h_{r}}$ são dois a dois disjuntos, $\mathcal{B}$ é um conjunto linearmente independente. Assim, precisamos apenas mostrar que $\mathcal{B}$ é também gerador de $\mathcal{D}$.

Como

$$
\begin{aligned}
\eta_{h_{i}}^{q} & =\left(\sum_{x \in \mathcal{S}_{h_{i}}} x\right)^{q} \\
& =\left(x+x^{q}+\cdots+x^{q^{t_{h_{i}}-1}}\right)^{q} \\
& =x^{q}+x^{q^{2}}+\cdots+x^{q^{t_{h_{i}}}} \\
& =\left(\sum_{x \in \mathcal{S}_{h_{i}}} x\right) \\
& =\eta_{h_{i}}, \quad 1 \leq i \leq r .
\end{aligned}
$$

Segue, do Lema 2.1.1, que $\eta_{h_{i}} \in \mathcal{D}$.

Portanto, $\mathcal{D} \subset \mathcal{B}$.

Seja $\alpha \in \mathcal{D}=\oplus_{i=1}^{r} \mathbb{F} e_{i}$. Se $\alpha=\sum_{h \in A} \alpha_{h} h$ segue, do Lema 2.1.3, que para cada $h \in A$, temos que

$$
\alpha_{h}=\alpha_{h^{q}}=\cdots=\alpha_{h^{q^{t}}-1}
$$

e conseqüentemente

$$
\alpha=\sum_{h \in \mathcal{T}} \alpha_{h} \eta_{h}
$$

Sabemos do Teorema de Perlis e Walker 1.4.8 que o número de componentes simples da álgebra de grupo racional de um grupo abeliano finito $A$ é igual ao número de subgrupos cíclicos de $A$ e também o número de seus fatores cíclicos. 
Note que, se $x \in \mathcal{S}_{h}$ então $x=h^{q^{j}}$, para algum $j$. Como $\operatorname{mdc}(q, o(h))=1$ segue que $\langle x\rangle=\langle h\rangle$. Portanto, cada $q$-classe ciclotômica $\mathcal{S}_{h}$ é um subconjunto do conjunto $\mathcal{G}_{h}$ de todos os geradores do grupo cíclico $\langle h\rangle$. Assim, é claro que o número de subgrupos cíclicos de $A$ é uma cota inferior para o número de componentes simples e que esta cota é obtida se e somente se $\mathcal{S}_{h}=\mathcal{G}_{h}$, para toclo $h \in A$.

Para os inteiros $r$ e $m$, denotaremos por $\bar{r} \in \mathbb{Z}_{m}$ a imagem de $r$ no anel dos inteiros módulo $m$. Então

$$
\mathcal{G}_{h}=\left\{h^{r} \mid \operatorname{mdc}(r, o(h))=1\right\}=\left\{h^{r} \mid \bar{r} \in U\left(\mathbb{Z}_{o(h)}\right)\right\}
$$

e temos o seguinte.

Teorema 2.2.2. Sejam $\mathbb{F}$ um corpo finito com $|\mathbb{F}|=q$ elementos e $A$ um grupo abeliano finito de expoente e, tal que $m d c(q,|A|)=1$. Então $\mathcal{S}_{h}=\mathcal{G}_{h}$, para todo $h \in A$ se e somente se $U\left(\mathbb{Z}_{e}\right)$ é um grupo cíclico gerado por $\bar{q} \in \mathbb{Z}_{e}$.

Demonstração: Assuma que $U\left(\mathbb{Z}_{\epsilon}\right)$ é um grupo cíclico gerado por $\bar{q} \in$ $\mathbb{Z}_{e}$. Para um elemento $h \in A$ temos que $o(h) \mid e$.

Seja $\pi$ o epimorfismo natural de $\mathbb{Z}_{e}$ em $\mathbb{Z}_{o(h)}$. Como $\bar{q} \in \mathbb{Z}_{e}$ é um gerador do grupo cíclico $U\left(\mathbb{Z}_{e}\right)$ tem-se que $\pi(\bar{q}) \in \mathbb{Z}_{o(h)}$ é um gerador do grupo cíclico $U\left(\mathbb{Z}_{o(h)}\right)$.

Para cada elemento $x \in \mathcal{G}_{h}$ temos que $x=h^{r}$, para algum inteiro positivo $r$ tal que $\bar{r} \in U\left(\mathbb{Z}_{o(h)}\right)$. Então, $\bar{r}=\pi(\bar{q})^{j}$ para algum $j$ e $x=h^{\pi(\bar{q})^{j}} \in \mathcal{S}_{h}$. Isto mostra que $\mathcal{G}_{h} \subset \mathcal{S}_{h}$. Portanto, $\mathcal{S}_{h}=\mathcal{G}_{h}$.

Reciprocamente, suponhamos que $\mathcal{S}_{h}=\mathcal{G}_{h}$, para todo $h \in A$.

Se $A$ é um grupo abeliano de expoente $e$, então existe um elemento $h_{0} \in A$ de ordem $e$ e, em particular, $\mathcal{S}_{h_{0}}=\mathcal{G}_{h_{0}}$.

Portanto, para cada inteiro positivo $r$ tal que $\bar{r} \in U\left(\mathbb{Z}_{e}\right)$ temos que $h_{0}^{r} \in \mathcal{G}_{h_{0}}=\mathcal{S}_{h_{0}}$, logo existe algum inteiro $j$ tal que $r=q^{j}$. Portanto, $\bar{q}$ gera $U\left(\mathbb{Z}_{e}\right)$.

É bem conhecido que $U\left(\mathbb{Z}_{e}\right)$ é um grupo cíclico se, e somente se, $e=$ $2,4, p^{n}$ ou $2 p^{n}$, onde $p$ é um inteiro primo ímpar e $n$ é um inteiro positivo. Para maiores detalhes consulte [15, pag. 63, Teorema 21].

Corolário 2.2.3. Sejam $\mathbb{F}$ um corpo finito com $|\mathbb{F}|=q$ elementos, $A$ um grupo abeliano finito de expoente e, tal que $\operatorname{malc}(q,|A|)=1$. Então $\mathcal{S}_{h}=\mathcal{G}_{h}$, para todo $h \in A$ se e somente se uma das seguintes afirmaçoes acontece. 
(i) $e=2$ e q é ímpar;

(ii) $e=4$ e $q \equiv 3 \quad(\bmod 4)$;

(iii) $e=p^{n}$ e $o(q)=\phi\left(p^{n}\right)$ em $U\left(\mathbb{Z}_{p^{n}}\right)$;

(iv) $e=2 p^{n}$ e o $(q)=\phi\left(p^{n}\right)$ em $U\left(\mathbb{Z}_{2 p^{n}}\right)$.

\section{Demonstração:}

(i) Sejam $e=2$. Um gerador do grupo cíclico $U\left(\mathbb{Z}_{2}\right)$ é um elemento $t \in \mathbb{Z}_{+}$ tal que $m d c(t, 2)=1$, isto é, todo inteiro positivo ímpar satisfaz essa condição. Logo, se $q$ é ímpar então $\bar{q}$ é um gerador do grupo cíclico $U\left(\mathbb{Z}_{2}\right)$.

(ii) Considere o grupo cíclico $U\left(\mathbb{Z}_{4}\right)$. O único gerador de $U\left(\mathbb{Z}_{4}\right)=\{\overline{1}, \overline{3}\}$ é $\bar{q}=\overline{3}$. Logo, $\bar{q}$ gera $U\left(\mathbb{Z}_{4}\right)$ se e somente se $q \equiv 3 \quad(\bmod 4)$.

(iii) Considere o grupo cíclico $U\left(\mathbb{Z}_{p^{n}}\right)$. Então $\bar{q}$ gera $U\left(\mathbb{Z}_{p^{n}}\right)$ se e somente se $m d c\left(q, p^{n}\right)=1$. Segue, do Teorema de Euler, que $q^{\phi\left(p^{n}\right)} \equiv 1 \quad\left(\bmod p^{n}\right)$, isto é, $o(q)=\phi\left(p^{n}\right)$ em $U\left(\mathbb{Z}_{p^{n}}\right)$.

(iv) Considere o grupo cíclico $U\left(\mathbb{Z}_{2 p^{n}}\right)$. Então $\bar{q}$ gera $U\left(\mathbb{Z}_{2 p^{n}}\right)$ se e somente se $m d c\left(q, 2 p^{n}\right)=1$. Segue, do Teorema de Euler, que $q^{\phi\left(2 p^{n}\right)} \equiv 1$ $\left(\bmod 2 p^{n}\right)$. Como $\phi\left(2 p^{n}\right)=\phi(2) \phi\left(p^{n}\right)=\phi\left(p^{n}\right)$ segue que $q^{\phi\left(p^{n}\right)} \equiv 1$ $\left(\bmod 2 p^{n}\right)$, isto é, $o(q)=\phi\left(p^{n}\right)$ em $U\left(\mathbb{Z}_{2 p^{n}}\right)$. 


\section{Capítulo 3}

\section{Conceitos Básicos da Teoria de Códigos}

\subsection{Códigos Corretores de Erros}

Seja $\mathcal{A}$ um conjunto finito qualquer que chamaremos de alfabeto. $\mathrm{O}$ número de elementos de $\mathcal{A}$ será denotado por q.

Os elementos de $\mathcal{A}$ serão chamados de letras ou dígitos. Uma seqüência de elementos de $\mathcal{A}$ diz-se uma palavra. O comprimento de uma palavra é o número de letras que compõe a palavra.

Consideremos $\mathcal{A}^{n}$ o conjunto de todas as palavras de comprimento $n$ sobre $\mathcal{A}$, isto é, $\mathcal{A}^{n}=\left\{\left(c_{0}, \ldots, c_{n-1}\right): c_{i} \in \mathcal{A}, 0 \leq i \leq n-1\right\}$.

Definição 3.1.1. Um código é um subconjunto próprio qualquer de $\mathcal{A}^{n}$, para algum número natural $n$.

Neste trabalho consideraremos apenas códigos em que todas as palavras têm o mesmo comprimento.

Às vezes, para enfatizarmos o fato de que $\mathcal{A}$ tem $q$ elementos, dizemos que um código $\mathcal{C} \subset \mathcal{A}^{n}$ é um código de bloco q-ário.

Algumas vezes, denotaremos a palavra $\left(c_{0}, c_{1}, \ldots, c_{n-1}\right)$ simplesmente por $c_{0} c_{1} \ldots c_{n-1}$, quando o significado for claro no contexto.

Exemplo 3.1.2. Seja $\mathcal{A}=\{0,1\}$. Considere o conjunto $\mathcal{C}=\{00000,01011$, $10110,11101\} \subset \mathcal{A}^{5}$, logo pela definição, $\mathcal{C}$ é um código de bloco binário. 
Definição 3.1.3. Dados dois elementos $x$, y pertencentes a $\mathcal{A}^{n}$, a distância de Hamming entre $x, y$ é o número de coordenadas em que $x$ e $y$ diferem

$$
d(x, y)=\left|\left\{i: x_{i} \neq y_{i}, 1 \leq i \leq n\right\}\right| .
$$

Exemplo 3.1.4. Se $x=01011$ e $y=11101$ então $d(x, y)=3$.

Pode-se demonstrar facilmente que o par $\left(\mathcal{A}^{n}, d\right.$ ) é um espaço métrico, onde $d$ é a distância de Hamming.

Definição 3.1.5. Seja $\mathcal{C}$ um código. $A$ distância mínima de $\mathcal{C}$ é o número

$$
d=\min \{d(u, v): u, v \in \mathcal{C} \text { e } u \neq v\}
$$

Exemplo 3.1.6. Vamos achar a distância mínima de $\mathcal{C}$, onde $\mathcal{C}$ é clado no Exemplo 3.1.2. Assim,

$$
\begin{aligned}
& \mathrm{d}(00000,01011)=3 ; \quad \mathrm{d}(00000,10110)=3 ; \quad \mathrm{d}(00000,11101)=4 ; \\
& \mathrm{d}(01011,10110)=4 ; \quad \mathrm{d}(01011,11101)=3 ; \quad \mathrm{d}(10110,11101)=3 ;
\end{aligned}
$$

portanto, a distância mínima de $\mathcal{C}$ é 3 .

Definição 3.1.7. Dado um elemento $x \in \mathcal{A}^{n}$ e um número real $r>0$

(i) Define-se o disco de centro em $x$ e raio $r$, como sendo o conjunto

$$
D(x, r)=\left\{u \in \mathcal{A}^{n}: d(u, x) \leq r\right\} .
$$

(ii) Define-se a esfera de centro em $x$ e raio $r$, como sendo o conjunto

$$
S(x, r)=\left\{u \in \mathcal{A}^{n}: d(u, x)=r\right\} .
$$

Definição 3.1.8. Seja $\mathcal{C}$ um código com distância mínima d, chamamos de capacidade de correção de $\mathcal{C}$ ao número

$$
\kappa=\left[\frac{d-1}{2}\right]
$$

onde $[x]$ representa a parte inteira do número real $x$.

Lema 3.1.9. Seja $\mathcal{C}$ um código com distância mínima d. Se c e c' são palavras distintas de $\mathcal{C}$ então

$$
D(c, \kappa) \bigcap D\left(c^{\prime}, \kappa\right)=\emptyset .
$$


O próximo lema nos diz quantas palavras há em cada disco de centro em $c \in \mathcal{A}^{n}$ e raio $r$.

Lema 3.1.10. Para todo $c \in \mathcal{A}^{n}$ e todo número natural $r>0$, temos que

$$
|D(c, r)|=\sum_{i=0}^{r}\left(\begin{array}{c}
n \\
i
\end{array}\right)(q-1)^{i} .
$$

Teorema 3.1.11. Seja $\mathcal{C}$ um código com distância mínima d. Então $\mathcal{C}$ pode detectar até $d-1$ erros e corrigir até $\kappa=\left[\frac{d-1}{2}\right]$ erros.

Definição 3.1.12. Um código $\mathcal{C}$ diz-se um código corretor de erros com capacidade $\kappa$ se

Para todo $x, y \in \mathcal{C}$ se $x \neq y$ então $d(x, y) \geq 2 \kappa+1$.

Exemplo 3.1.13. Utilizando novamente o código $\mathcal{C}$ do Exemplo 3.1.2, temos que $\mathcal{C}$ é um código corretor de erros com capacidade de correção 1 e $\mathcal{C}$ pode cletectar até 2 erros.

Definição 3.1.14. Seja $\mathcal{C} \subset \mathcal{A}^{n}$ um código com distância mínima d e seja $\kappa=\left[\frac{d-1}{2}\right]$. O código $\mathcal{C}$ será dito perfeito se

$$
\bigcup_{c \in \mathcal{C}} D(c, \kappa)=\mathcal{A}^{n}
$$

Um código sobre um alfabeto $\mathcal{A}$ possui três parâmetros fundamentais (n, $\mathrm{M}, d$ ), que são respectivamente, o seu comprimento (o número $n$ corresponde à dimensão do espaço ambiente $\mathcal{A}^{n}$ onde $\mathcal{C}$ se encontra), o seu número de elementos e a sua distância mínima.

Proposição 3.1.15 (Cota de Hamming). Se C é um código com parâmetros $\left[n, q^{k}, d\right]$ e $\kappa=[(d-1) / 2]$ então

$$
q^{k} \leq \frac{q^{n}}{\sum_{i=0}^{\kappa}\left(\begin{array}{c}
n \\
i
\end{array}\right)(q-1)^{i}}
$$

valendo a igualdade se. e somente se, $\mathcal{C}$ é perfeito. 


\section{CAPÍTULO 3. CONCEITOS BÁSICOS DA TEORIA DE CÓDIGOS}

O objetivo dos códigos corretores de erros é acrescentar dados adicionais a mensagem que iremos transmitir ou armazenar de forma que nos permita recuperá-la detectando e corrigindo possíveis erros. O processo de adicionar dados a mensagem é chamado de codificação. E o processo de recuperação da mensagem é chamado de decodificação.

Os clados que adicionaremos a mensagem chamaremos de dígitos de verificação ou redundância e são construidos em função dos dígitos da mensagem e estes, por sula vez, serão denotados por dígitos de informação.

Exemplo 3.1.16. Sejam $\mathcal{A}=\{0,1\}$ e $\mathcal{A}^{5}=\left\{\left(x_{0}, x_{1}, x_{2}, x_{3}, x_{4}\right) \mid x_{i} \in \mathcal{A}\right.$, $0 \leq i \leq 4\}$. Vamos construir um código $\mathcal{C} \subset \mathcal{A}^{5}$.

Sejam $x_{0}, x_{1}, x_{2} \in \mathcal{A}$. Vamos construir $x_{3}$ e $x_{4}$ em função de $x_{0}, x_{1}, x_{2}$, isto é, vamos codificar a mensagem $x_{0} x_{1} x_{2}$.

Defino $x_{3}=x_{0}+x_{2}$ e $x_{4}=x_{1}+x_{2}$.

Logo, $\left(x_{0}, x_{1}, x_{2}, x_{3}, x_{4}\right) \in \mathcal{C} \subset \mathcal{A}^{5}$. Assim,

$$
\mathcal{C}=\{00000,00111,01001,10010,01110,11011,10101,11100\} .
$$

Note que, os três primeiros dígitos são de informações e os dois últimos de verificações.

\subsection{Códigos Lineares}

Esta seção e a de códigos cíclicos foi elaborada com a co-autoria de meu orientador.

Iremos apresentar as definições e propriedades destes tópicos um pouco diferente das encontradas na literatura da teoria dos códigos corretores de erros. Nossa intenção é usar a notação de álgebra linear tal como é familiar aos estudantes de matemática.

Com pequenas adlaptações, as demonstrações dos lemas, proposições, corolários e teoremas que apresentaremos nesta seção podem ser encontrados em livros básicos de Teoria de Códigos tais como: [2], [14], [16], [22], [24].

Assumiremos, a partir de agora, que o alfabeto para codificação será sempre o corpo finito $\mathbb{F}$ com $q$ elementos.

Definição 3.2.1. Um código $\mathcal{C} \subset \mathbb{F}^{n}$ será chamado de código linear se for um subespaço vetorial de $\mathbb{F}^{n}$. Se $\mathcal{C}$ tem dimensão $k$ sobre $\mathbb{F}$ dizemos que $\mathcal{C}$ é um $(n, k)$-código linear e se $\mathcal{C}$ tem distância mínima d, dizemos que $\mathcal{C}$ é um $(n, k, d)$-código linear. 
Exemplo 3.2.2. Seja $\mathbb{F}$ o corpo finito com dois elementos. Vamos mostrar que o código corretor de erros $\mathcal{C}$ do Exemplo 3.1.2 é um código linear. Para isto, precisamos mostrar que para toclo $u, v \in \mathcal{C}$ e toclo $\lambda \in \mathbb{F}$ temos que $u+\lambda v \in$ $\mathcal{C}$. Lembremos que $\mathcal{C}=\{00000,01011,10110,11101\} \subset \mathbb{F}^{5}$. Assim, $\lambda \in\{0,1\}$. Se $\lambda=0$ então $u+\lambda v=u \in \mathcal{C}$, para todo $u \in \mathcal{C}$. Se $\lambda=1$ temos que a soma de quaisquer $u, v \in \mathcal{C}$ pertencem a $\mathcal{C}$. De fato,

$$
\begin{aligned}
& 01011+10110=11101 \in \mathcal{C} \\
& 01011+11101=10110 \in \mathcal{C} \\
& 10110+11101=01011 \in \mathcal{C}
\end{aligned}
$$

Portanto, $\mathcal{C}$ é um subespaço vetorial de $\mathbb{F}^{5}$. A dimensão de $\mathcal{C}$ sobre $\mathbb{F}$ é 2 . Assim, $\mathcal{C}$ é um $(5,2,3)$-código linear.

Definição 3.2.3. Dado $u \in \mathbb{F}^{n}$ chama-se peso de $u$ ao número de coordenadas não nulas de u, isto é:

$$
\omega(u)=\left|\left\{i: u_{i} \neq 0\right\}\right|
$$

em outras palavras, temos que

$$
\omega(u)=d(u, 0)
$$

onde d é a distância mínima de Hamming de $\mathcal{C}$.

Exemplo 3.2.4. Se $x=01011$ então $\omega(x)=3$.

Definição 3.2.5. O peso de um código linear $\mathcal{C}$ é o inteiro

$$
\omega(\mathcal{C})=\min \{\omega(u): u \in \mathcal{C} \text { e } u \neq 0\}
$$

Proposição 3.2.6. Seja $\mathcal{C} \subset \mathbb{F}^{n}$ um código linear com distância mínima d. Temos que

(i) $\forall x, y \in \mathbb{F}^{n}, d(x, y)=\omega(x-y)$.

(ii) $d=\omega(\mathcal{C})$.

Há várias maneiras de descrevermos 1 m código linear $\mathcal{C}$. Apresentaremos duas delas neste trabalho. 


\section{CAPÍTULO 3. CONCEITOS BÁSICOS DA TEORIA DE CÓDIGOS}

Consicleremos a base canônica $\left\{e_{1}, \ldots, e_{n}\right\}$ de $\mathbb{F}^{n}$, para algum natural $n$. Assim, todo vetor $v \in \mathbb{F}^{n}$ tem a forma

$$
v=v_{1} e_{1}+\cdots+v_{n} e_{n} \text {, onde } v_{i} \in \mathbb{F}, \forall i=0, \ldots, n \text {. }
$$

Seja $v \in \mathbb{F}^{n}$, este vetor será escrito como vetor-linha ou vetor-coluna quando for conveniente, e o significado ficará claro no contexto. a $\mathbb{F}^{k}$.

Seja $\mathcal{C} \subset \mathbb{F}^{n}$ um código linear de dimensão $k$ sobre $\mathbb{F}$. Então $\mathcal{C}$ é isomorfo

Assim, podemos definir uma função linear injetora

$$
\nu: \mathbb{F}^{k} \longrightarrow \mathbb{F}^{n} \text { tal que } \operatorname{Im}(\nu)=\mathcal{C} .
$$

Por exemplo, dada uma base $\left\{c_{1}, \ldots, c_{k}\right\}$ de $\mathcal{C}$ e consideremos a base canônica $\left\{e_{1}, \ldots, e_{k}\right\}$ de $\mathbb{F}^{k}$.

Defino $\nu: \mathbb{F}^{k} \longrightarrow \mathbb{F}^{n}$ por $\nu\left(e_{i}\right)=c_{i}$, para $0 \leq i \leq k$.

Esta aplicação pode ser visualizada no seguinte diagrama:

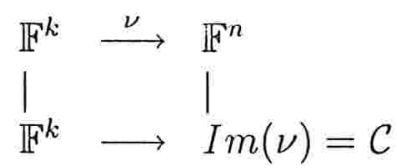

Vamos encontrar a matriz $G$ que representa a transformação linear $\nu$ nas bases canônicas destes espaços.

Considere $B=\left\{f_{1}, \ldots, f_{n}\right\}$ a base canônica de $\mathbb{F}^{n}$.

Escrevendo os elementos da base de $\mathcal{C}$ em função da base canônica, temos

$$
\left\{\begin{array}{ccc}
c_{1}= & b_{11} f_{1}+b_{21} f_{2}+\ldots+b_{n 1} f_{n} \\
c_{2}= & b_{12} f_{1}+b_{22} f_{2}+\ldots+b_{n 2} f_{n} \\
\vdots & \vdots \vdots \\
c_{k}= & b_{1 k} f_{1}+b_{2 k} f_{2}+\ldots+b_{n k} f_{n}
\end{array}\right.
$$

para certos $b_{i j} \in \mathbb{F}$.

Como $\nu\left(e_{i}\right)=c_{i}=b_{1 i} f_{1}+\ldots+b_{n i} f_{n}, 0 \leq i \leq k$, a matriz de $\nu$ nas respectivas bases canônicas é

$$
G=\left[\begin{array}{cccc}
b_{11} & b_{12} & \cdots & b_{1 k} \\
b_{21} & b_{22} & \cdots & b_{2 k} \\
\vdots & \vdots & & \vdots \\
b_{n 1} & b_{n 2} & \cdots & b_{n k}
\end{array}\right]
$$


Consiclere $v \in \mathbb{F}^{k}$. Então $v=\left(v_{1}, \ldots, v_{k}\right)$ onde $v_{i} \in \mathbb{F}$. A matriz de $\nu$ se escreve, em coordenadas, pela equação matricial

$$
\left[\begin{array}{cccc}
b_{11} & b_{12} & \cdots & b_{1 k} \\
b_{21} & b_{22} & \cdots & b_{2 k} \\
\vdots & \vdots & & \vdots \\
b_{n 1} & b_{n 2} & \cdots & b_{n k}
\end{array}\right]\left[\begin{array}{c}
v_{1} \\
v_{2} \\
\vdots \\
v_{k}
\end{array}\right]=\left[\begin{array}{c}
d_{1} \\
d_{2} \\
\vdots \\
d_{n}
\end{array}\right]
$$

Denotamos por $G$ a matriz $\left(b_{i j}\right)_{i, j} \in \mathbb{M}_{n \times k}(\mathbb{F})$.

Como $\operatorname{Im}(\nu)=\mathcal{C}$ temos que cada vetor-coluna de $G$ pertence ao código $\mathcal{C}$, ou seja, $\mathcal{C}$ é o subespaço gerado pelas colunas de $G$, que formam, na realidade, uma base de $\mathcal{C}$. Os elementos de $\mathcal{C}$ são todas as palavras $y$ da forma $G x=y, \forall x \in \mathbb{F}^{k}$.

Definição 3.2.7. Uma matriz $G \in \mathbb{M}_{n \times k}(\mathbb{F})$ cujas colunas formam uma base para $\mathcal{C}$ diz-se matriz de codificação de $\mathcal{C}$ nas bases canônicas.

Note que, para cada base escolhida para $\mathcal{C}$ temos uma matriz de codificação $G$.

Exemplo 3.2.8. Seja $\mathbb{F}$ o corpo finito com dois elementos. Considere a transformação linear injetora

$$
\begin{aligned}
& \nu: \mathbb{F}^{3} \quad \longrightarrow \mathbb{F}^{5} \\
& \left(x_{1}, x_{2}, x_{3}\right) \longmapsto\left(x_{1}, x_{3}, x_{1}+x_{2}, x_{2}+x_{3}, x_{2}\right)
\end{aligned}
$$

Seja $\mathcal{C}=\operatorname{Im}(\nu)$. $\mathbb{F}^{5}$.

Sejam $\left\{e_{1}, e_{2}, e_{3}\right\}$ a base canônica de $\mathbb{F}^{3}$ e $\left\{f_{1}, f_{2}, f_{3}, f_{4}, f_{5}\right\}$ a base canônica de

Vamos encontrar uma matriz $G$ que representa a transformação linear $\nu$.

Assin,

$$
\begin{aligned}
& \nu\left(e_{1}\right)=(1,0,1,0,0)=1 f_{1}+0 f_{2}+1 f_{3}+0 f_{4}+0 f_{5} \\
& \nu\left(e_{2}\right)=(0,0,1,1,1)=0 f_{1}+0 f_{2}+1 f_{3}+1 f_{4}+1 f_{5} \\
& \nu\left(e_{3}\right)=(0,1,0,1,0)=0 f_{1}+1 f_{2}+0 f_{3}+1 f_{4}+0 f_{5}
\end{aligned}
$$

Portanto, uma matriz de codificação $G$ é da forma

$$
G=\left[\begin{array}{lll}
1 & 0 & 0 \\
0 & 0 & 1 \\
1 & 1 & 0 \\
0 & 1 & 1 \\
0 & 1 & 0
\end{array}\right]
$$


Exemplo 3.2.9. Seja $\mathbb{F}$ o corpo finito com dois elementos. Considere o código linear binário $\mathcal{C} \subset \mathbb{F}^{5}$ definido pela transformação linear injetora

$$
\begin{aligned}
& \nu: \mathbb{F}^{3} \quad \longrightarrow \mathbb{F}^{5} \\
& \left(x_{1}, x_{2}, x_{3}\right) \longmapsto\left(x_{1}, x_{2}, x_{3}, x_{1}+x_{3}, x_{1}+x_{2}\right)
\end{aligned}
$$
$\mathbb{F}^{5}$.

Sejam $\left\{e_{1}, e_{2}, e_{3}\right\}$ a base canônica de $\mathbb{F}^{3}$ e $\left\{f_{1}, f_{2}, f_{3}, f_{4}, f_{5}\right\}$ a base canônica de

Vamos encontrar uma matriz $G$ que representa a transformação linear $\nu$.

Assim,

$$
\begin{aligned}
& \nu\left(e_{1}\right)=(1,0,0,1,1)=1 f_{1}+0 f_{2}+0 f_{3}+1 f_{4}+1 f_{5} \\
& \nu\left(e_{2}\right)=(0,1,0,0,1)=0 f_{1}+1 f_{2}+0 f_{3}+0 f_{4}+1 f_{5} \\
& \nu\left(e_{3}\right)=(0,0,1,1,0)=0 f_{1}+0 f_{2}+1 f_{3}+1 f_{4}+0 f_{5}
\end{aligned}
$$

Portanto, uma matriz de codificação $G$ é da forma

$$
G=\left[\begin{array}{lll}
1 & 0 & 0 \\
0 & 1 & 0 \\
0 & 0 & 1 \\
1 & 0 & 1 \\
1 & 1 & 0
\end{array}\right]
$$

Seja $v \in \mathcal{C}$. Observe que as três primeiras coordenadas são os dígitos de informação, logo este código tem uma decodificação muito simples. Por exemplo, se recebermos a palavra (10101) então a mensagem enviada é (101).

Uma codificação que aplica uma mensagem $x=\left(x_{1}, \ldots, x_{k}\right)$ em uma palavra do código $c=\left(c_{1}, \ldots, c_{n}\right)$ tal que existem coordenadas $i_{1}, \ldots, i_{k}$ oncle $x_{1}=c_{i_{1}}, \ldots, x_{k}=c_{i_{k}}$, diz-se codificação sistemática.

Um código diz-se separável se admite uma codificação sistemática.

Matrizes de codificação com a forma apresentada no exemplo acima recebe um nome especial na teoria dos códigos.

Definição 3.2.10. Diz-se que uma matriz de codificação $G$ de um código $\mathcal{C}$ está na forma padrão se ela é da forma $G=\left(\begin{array}{c}I d_{k} \\ A\end{array}\right)$, onde $I d_{k} \in \mathbb{M}_{k \times k}(\mathbb{F})$ é a matriz identidade e $A \in \mathbb{M}_{(n-k) \times k}(\mathbb{F})$.

Outra maneira de descrevermos o código linear $\mathcal{C}$ é através de uma transformação linear sobrejetora $\pi: \mathbb{F}^{n} \longrightarrow \mathbb{F}^{n-k}$ tal que $\operatorname{Ker}(\pi)=\mathcal{C}$. 
Por exemplo, dado uma base $\left\{c_{1}, \ldots, c_{k}\right\}$ de $\mathcal{C}$ e ampliando a uma base $\left\{c_{1}, \ldots, c_{k}, v_{1}, \ldots, v_{n-k}\right\}$ de $\mathbb{F}^{n}$.

Seja $v \in \mathbb{F}^{n}$. Logo, $v$ pode ser escrito como

$$
v=\lambda_{1} c_{1}+\cdots+\lambda_{k} c_{k}+\lambda_{k+1} v_{1}+\cdots+\lambda_{n} v_{n-k}
$$

onde $\lambda_{i} \in \mathbb{F}, 1 \leq i \leq n$.

Defino $\pi: \mathbb{F}^{n} \longrightarrow \mathbb{F}^{n-k}$ por

$$
v \mapsto v^{\prime}=\lambda_{k+1} v_{1}+\cdots+\lambda_{n} v_{n-k}
$$

Esta aplicação pode ser visualizada através do seguinte diagrama

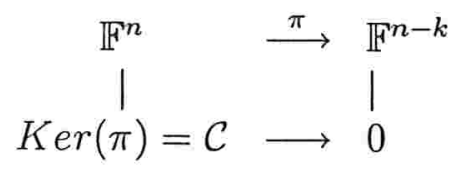

Denotaremos por $H=\left(h_{i j}\right)_{i, j} \in \mathbb{M}_{(n-k) \times n}(\mathbb{F})$ a matriz de posto $(n-k)$ que representa a transformação linear $\pi$ nas bases canônicas destes espaços.

Como $\operatorname{Ker}(\pi)=\mathcal{C}$ temos que o conjunto de todas as palavras $x \in \mathbb{F}^{n}$ satisfazendo $H x=0$ formam o código linear $\mathcal{C}$.

Definição 3.2.11. A matriz $H$ construida acima diz-se uma matriz de teste do código linear $\mathcal{C}$.

Assim, um código linear $\mathcal{C}$ também pode ser definido a partir de uma matriz de teste.

Exemplo 3.2.12. Seja $\mathbb{F}$ o corpo finito com dois elementos. Considere a transformação linear sobrejetora

$$
\begin{aligned}
& \pi: \mathbb{F}^{3} \quad \longrightarrow \mathbb{F}^{2} \\
& \left(x_{1}, x_{2}, x_{3}\right) \longmapsto\left(x_{1}+x_{2}, x_{3}\right)
\end{aligned}
$$

tal que $\mathcal{C}=\operatorname{Ker}(\pi)=\left\{\left(x_{1}, x_{1}, 0\right) \mid x_{1} \in \mathbb{F}\right\}$.

Agora, considere as bases canônicas $\left\{e_{1}, e_{2}, e_{3}\right\}$ e $\left\{f_{1}, f_{2}\right\}$, respectivamente de $\mathbb{F}^{3}$ e $\mathbb{F}^{2}$.

Vamos achar a matriz $H$ que representa a transformação linear $\pi$.

Assim,

$$
\begin{aligned}
& \pi\left(e_{1}\right)=\pi(100)=1 f_{1}+0 f_{2} \\
& \pi\left(e_{2}\right)=\pi(010)=1 f_{1}+0 f_{2} \\
& \pi\left(e_{3}\right)=\pi(001)=0 f_{1}+1 f_{2}
\end{aligned}
$$


40 CAPÍTULO 3. CONCEITOS BÁSICOS DA TEORIA DE CÓDIGOS

Portanto, a matriz $H$ tem a forma

$$
H=\left[\begin{array}{lll}
1 & 1 & 0 \\
0 & 0 & 1
\end{array}\right]
$$

Observe que ao tomarmos um vetor qualquer $y \in \mathbb{F}^{3}$, para verificarmos se ele pertence ao código $\mathcal{C}$, precisamos verificar se a condição $H y=0$ é satisfeita.

Dado $y=(1,1,1), z=(1,1,0) \in \mathbb{F}^{3}$.

Como

$$
H y=\left(\begin{array}{l}
0 \\
1
\end{array}\right) \text { e } H z=\left(\begin{array}{l}
0 \\
0
\end{array}\right)
$$

temos que $y \notin \mathcal{C}$ e $z \in \mathcal{C}$.

Exemplo 3.2.13. Vamos encontrar uma matriz de teste do código $\mathcal{C}$ do Exemplo 3.2.8.

Temos que $c_{1}=(10100), c_{2}=(00111), c_{3}=(01010)$ formam uma base de $\mathcal{C}$. Vamos ampliar esta base e determinar uma base para $\mathbb{F}^{5}$.

Se tomarmos $c_{4}=(00010)$ e $c_{5}=(00001)$ então o conjunto $B=\left\{c_{1}, c_{2}\right.$, $\left.c_{3}, c_{4}, c_{5}\right\}$ é uma base de $\mathbb{F}^{5}$.

Defino $\pi: \mathbb{F}^{5} \longrightarrow \mathbb{F}^{2}$ por $\left\{\begin{array}{l}\pi\left(c_{i}\right)=(00) \quad \text { se } 1 \leq i \leq 3 \\ \pi\left(c_{4}\right)=(10) \\ \pi\left(c_{5}\right)=(01)\end{array}\right.$

Vamos escrever os elementos da base canônica de $\mathbb{F}^{5}$ na base $B$.

$$
\begin{aligned}
& (10000)=1 c_{1}+1 c_{2}+0 c_{3}+1 c_{4}+1 c_{5} \\
& (01000)=0 c_{1}+0 c_{2}+1 c_{3}+1 c_{4}+0 c_{5} \\
& (00100)=0 c_{1}+1 c_{2}+0 c_{3}+1 c_{4}+1 c_{5} \\
& (00010)=0 c_{1}+0 c_{2}+0 c_{3}+1 c_{4}+0 c_{5} \\
& (00001)=0 c_{1}+0 c_{2}+0 c_{3}+0 c_{4}+1 c_{5}
\end{aligned}
$$

Assim,

$$
\begin{array}{ll}
\pi(10000)=\pi\left(c_{1}\right)+\pi\left(c_{2}\right)+\pi\left(c_{4}\right)+\pi\left(c_{5}\right) & =(10)+(01)=(11) \\
\pi(01000)=\pi\left(c_{3}\right)+\pi\left(c_{4}\right) & =(10) \\
\pi(00100)=\pi\left(c_{2}\right)+\pi\left(c_{4}\right)+\pi\left(c_{5}\right) & =(10)+(01)=(11) \\
\pi(00010)=\pi\left(c_{4}\right) & =(10) \\
\pi(00001)=\pi\left(c_{5}\right) & =(01)
\end{array}
$$

Portanto, uma matriz de teste é

$$
H=\left[\begin{array}{lllll}
1 & 1 & 1 & 1 & 0 \\
1 & 0 & 1 & 0 & 1
\end{array}\right]
$$


Vamos analisar como $\pi$ e $\nu$ se relacionam.

Agora, considere o diagrama

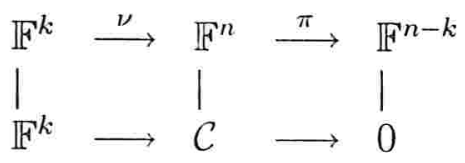

onde $\mathcal{C}=\operatorname{Im}(\nu)=\operatorname{Ker}(\pi)$.

Se $x \in \mathbb{F}^{k}$ temos que $\pi \circ \nu(x)=\pi(\nu(x))=0$, pois $\nu(x) \in \operatorname{Im}(\nu)=\mathcal{C}=$ $\operatorname{Ker}(\pi)$.

Em notação matricial, temos que $H G=0$.

Podemos definir um "produto" em $\mathbb{F}^{n}$ por analogia com o produto escalar dos espaços em $\mathbb{R}^{n}$ (onde $\mathbb{R}$ denota o corpo dos números reais).

Sejam $x=\left(x_{1}, \ldots, x_{n}\right)$ e $y=\left(y_{1}, \ldots, y_{n}\right)$ vetores em $\mathbb{F}^{n}$, define-se o produto escalar de $x$ e $y$ em $\mathbb{F}^{n}$ por

$$
x \cdot y=x_{1} y_{1}+\cdots+x_{n} y_{n}
$$

Se $x \cdot y=0$ então $x$ e $y$ dizem-se ortogonais. Em particular, note que os vetores-linhas de uma matriz de teste $H$ de um código $\mathcal{C}$ são ortogonais aos vetores-coluna de uma matriz de codificação $G$.

O produto escalar satisfaz as seguintes propriedades:

(i) $u \cdot v=v \cdot u, \quad \forall u, v \in \mathbb{F}^{n}$.

(ii) $(u+v) \cdot w=u \cdot w+v \cdot w, \quad \forall u, v, w \in \mathbb{F}^{n}$.

(iii) $(\lambda u) \cdot v=\lambda(u \cdot v), \quad \forall \lambda \in \mathbb{F}, \forall u, v \in \mathbb{F}^{n}$.

Note que não vale, $u \cdot u=0$ se e somente se $u=0$. Por exemplo, seja $\mathbb{F}$ o corpo finito com dois elementos, se $u=(110) \in \mathbb{F}^{3}$ tem-se que $u \cdot u=0$.

Definição 3.2.14. Seja C um (n, k)-código linear. Diz-se que o conjunto

$$
\mathcal{C}^{\perp}=\left\{x \in \mathbb{F}^{n} \mid x \cdot y=0, \forall y \in \mathcal{C}\right\}
$$

é o código dual de $\mathcal{C}$. 
É fácil provar que $\mathcal{C}^{\perp}$ é um subespaço vetorial de $\mathbb{F}^{n}$ e, portanto um código linear.

Sejam $\mathcal{C}$ um $(n, k)$-código linear com matriz de codificação $G$ e $y \in \mathcal{C}^{\perp}$. Então $y \cdot x=0, \quad \forall x \in \mathcal{C}$, em particular, $y$ é ortogonal a todos os elementos de uma base de $\mathcal{C}$, o que equivale a dizermos que, $y G=0$.

Assim, podemos definir o código dual $\mathcal{C}^{\perp}$ da seguinte forma

$$
\mathcal{C}^{\perp}=\left\{y \in \mathbb{F}^{n} \mid y G=0\right\} .
$$

Exemplo 3.2.15. Note que a matriz de teste $H$ do Exemplo 3.2.13 é uma matriz de codificação do código dual a $\mathcal{C}$.

Vamos estudar algumas propriedades gerais dos códigos lineares.

Uma questão natural é a seguinte: dado uma matriz de codificação $G$ de um código linear $\mathcal{C}$ como encontrar uma matriz de teste $H$ de $\mathcal{C}$ a partir de G.

Proposição 3.2.16. Seja $\mathcal{C} \subset \mathbb{F}^{n}$ um $(n, k)$-código linear com matriz de codificação $G=\left(\begin{array}{c}I d_{k} \\ A\end{array}\right)$, na forma padrão. Então

(i) $\operatorname{dim} \mathcal{C}^{\perp}=n-k$.

(ii) $H=\left(-A \mid I d_{n-k}\right)$ é uma matriz de codificação de $\mathcal{C}^{\perp}$.

(iii) $\left(\mathcal{C}^{\perp}\right)^{\perp}=\mathcal{C}$.

Note que o espaço dual sobre um corpo finito tem propriedades diferentes das propriedades usuais do espaço dual sobre o corpo dos números reais. Por exemplo, seja $V$ um espaço vetorial sobre $\mathbb{R}$ de dimensão finita, e considere $W$ um subespaço vetorial de $V$ então $W \cap W^{\perp}=\{0\}$. Isto não ocorre sempre no caso de um subespaço vetorial $S$ de $V$ de dimensão finita sobre o corpo finito $\mathbb{F}$, pois podemos ter $S \cap S^{\perp} \neq\{0\}$ e até $S=S^{\perp}$.

Exemplo 3.2.17. Seja $\mathbb{F}$ o corpo finito com dois elementos. Considere $\mathcal{C}$ um (4,2)-código linear definido por

$$
\mathcal{C}=\{0000,1100,0011,1111\}
$$

Vamos encontrar seu código dual $\mathcal{C}^{\perp}$, ou seja, queremos encontrar todas as palavras $y \in \mathbb{F}^{4}$ tais que $y \cdot x=0$, para todo $x \in \mathcal{C}$. Assim,

$$
\mathcal{C}^{\perp}=\{0000,1100,0011,1111\} .
$$

Portanto, temos que $\mathcal{C}=\mathcal{C}^{\perp}$. 
A matriz de teste de um código contém informação sobre o valor do peso $d$ do código linear.

Lema 3.2.18. Seja $H$ uma matriz de teste de um código $\mathcal{C}$. Se existe $v \in$ $\mathcal{C}$ tal que $\omega(v)=t$ então existem $t$ colunas de $H$ que são linearmente dependentes.

Lema 3.2.19. Seja $H$ uma matriz de teste de um código $\mathcal{C}$. Se existem $t$ colunas de $H$ que são linearmente dependentes então $\omega(\mathcal{C}) \leq t$.

Teorema 3.2.20. Seja $H$ uma matriz de teste de um código $\mathcal{C}$. Temos que o peso de $\mathcal{C}$ é igual a d se, e somente se, quaisquer $d-1$ colunas de $H$ são linearmente independentes e existem $d$ colunas de $H$ linearmente dependentes.

Lembremos, da seção 1.1, que quanto maior o peso de um código linear $\mathcal{C}$ melhor sua capacidade de correção. Assim, é conveniente termos uma estimativa para este.

Corolário 3.2.21 (Cota de Singleton). Os parâmetros $(n, k, d)$ de um código linear $\mathcal{C}$ (onde $d=\omega(\mathcal{C})$ ) satisfazem à desigualdade

$$
d \leq n-k+1
$$

Note que a cota de Singleton nos diz que um código linear $\mathcal{C}$ de dimensão $k$ em $\mathbb{F}^{n}$ tem peso máximo possível se $d=n-k+1$.

Um código diz-se código separável pela distância máxima ou MDS (maximum distance separable) se vale a igualdade $d=n-k+1$.

Exemplo 3.2.22. Vamos construir um código binário $\mathcal{C}$ de comprimento $6 \mathrm{com}$ três dígitos de informação e três dígitos de redundância. Denotaremos os dígitos de informação por $c_{1}, c_{2}, c_{3}$ e os dígitos de redundância por $c_{4}, c_{5}, c_{6}$. Escolhendo os dígitos de redundância através da regra:

$$
\begin{aligned}
& c_{4}=c_{1}+c_{2} \\
& c_{5}=c_{1}+c_{3} \\
& c_{6}=c_{2}+c_{3}
\end{aligned}
$$

em notação matricial, temos que

$$
\left[\begin{array}{lll}
1 & 0 & 0 \\
0 & 1 & 0 \\
0 & 0 & 1 \\
1 & 1 & 0 \\
1 & 0 & 1 \\
0 & 1 & 1
\end{array}\right]\left[\begin{array}{c}
c_{1} \\
c_{2} \\
c_{3}
\end{array}\right]=\left[\begin{array}{c}
c_{1} \\
c_{2} \\
c_{3} \\
c_{1}+c_{2} \\
c_{1}+c_{3} \\
c_{2}+c_{3}
\end{array}\right]
$$




\section{CAPÍTULO 3. CONCEITOS BÁSICOS DA TEORIA DE CÓDIGOS}

onde a matriz acima é uma matriz de codificação $G$ do código binário $\mathcal{C}$.

Se recebemos uma palavra $y=\left(y_{1}, y_{2}, y_{3}, y_{4}, y_{5}, y_{6}\right) \in \mathbb{F}^{6}$ para saber se $y \in \mathcal{C}$ devemos verificar se as equações de teste são satisfeitas:

$\left\{\begin{array}{l}y_{14}=y_{1}+y_{2} \\ y_{5}=y_{1}+y_{3} \\ y_{6}=y_{2}+y_{3}\end{array} \approx\left\{\begin{aligned} y_{1}+y_{2}+y_{1} & =0 \\ y_{1}+y_{3}+y_{5} & =0 \\ y_{2}+y_{3} & =y_{6}=0\end{aligned}\right.\right.$

em notação matricial temos

$$
\left[\begin{array}{llllll}
1 & 1 & 0 & 1 & 0 & 0 \\
1 & 0 & 1 & 0 & 1 & 0 \\
0 & 1 & 1 & 0 & 0 & 1
\end{array}\right]\left[\begin{array}{l}
y_{1} \\
y_{2} \\
y_{3} \\
y_{4} \\
y_{5} \\
y_{6}
\end{array}\right]=\left[\begin{array}{l}
0 \\
0 \\
0
\end{array}\right]
$$

ou seja, a matriz que aparece acima é uma matriz de teste do código $\mathcal{C}$, isto é,

$$
y \in \mathcal{C} \Leftrightarrow H y=0
$$

Através de uma matriz de teste $H$ podemos achar a distância mínima de $\mathcal{C}$. Note que quaisquer duas colunas de $H$ são linearmente independentes e as três primeiras colunas de $H$ são linearmente dependente, logo segue do Teorema 3.2 .20 que $d=3$. Como $n=6$ e $k=3$ temos que $n-k+1 \geq 4>d$, assim este código não é $M D S$.

Note que a matriz obtida é uma matriz de teste $H$ correspondente a uma matriz de codificação $G$ na forma padrão, tal como resulta da Proposição 3.2.16. Observe que o número de dígitos de redunclância de uma palavra é o número de linhas da matriz de teste $H$.

Observamos aqui que na literatura da teoria dos códligos corretores de erros costuma-se usar, ao invés da matriz de codificação $G$ que definimos, a matriz transposta dela $G^{t}$ cujas linhas, então, geram o código e que é denotada por matriz geradora.

Logo, uma matriz geradora $G^{t}$ esta na forma padrão se $G^{t}=\left(I d_{k} \mid A\right)$ onde $I d_{k} \in \mathbb{M}_{k \times k}(\mathbb{F})$ é a matriz identidade e $A \in \mathbb{M}_{k \times n-k}(\mathbb{F})$. Então, sua matriz cle teste fica cla forma $H=\left(-A^{t} \mid I d_{n-k}\right)$, onde $I d_{k} \in \mathbb{M}_{n-k \times n-k}(\mathbb{F})$ é a matriz idlentidade. 


\subsection{Códigos Cíclicos}

Enriquecendo a estrutura do espaço vetorial $\mathbb{F}^{n}$ encontramos uma classe de códigos lineares muito importante: os códigos cíclicos. Estes são amplamente utilizados nos sistemas digitais onde os circuitos eletrônicos estão cada vez mais sucetíveis a interferências do meio devido ao avanço das tecnologias de fabricação, que permite a diminuição de suas dimensões. Assim, quando pensamos em tecnologia digital, estamos empregando, implicitamente, os códigos cíclicos.

Nosso objetivo, a partir de agora, é estudar estes códigos.

$A$ partir de agora, $\mathbb{F}$ será sempre um corpo finito com q elementos e $G=\left\{1, a, \ldots, a^{n-1}\right\}, a^{n}=1$, um grupo cíclico de ordem $n$.

Consideremos a álgebra de grupo de $G$ sobre $\mathbb{F}$. Note que, em particular, $\mathbb{F} G$ é um $\mathbb{F}$-espaço vetorial de dimensão $n$. Assim, $\mathbb{F} G$ é um $\mathbb{F}$-espaço vetorial isomorfo a $\mathbb{F}^{n}$. Representaremos as coordenadas de $\mathbb{F}^{n}$ por $\left(x_{0}, \ldots, x_{n-1}\right)$.

Realizaremos este isomorfismo de espaços vetoriais através da transformação linear $\psi: \mathbb{F}^{n} \longrightarrow \mathbb{F} G$ definida por

$$
\left(b_{0}, b_{1}, \ldots, b_{n-1}\right) \mapsto \sum_{i=0}^{n-1} b_{i} a^{i} .
$$

Ou seja, podemos associar a cada palavra $b=\left(b_{0}, b_{1}, \ldots, b_{n-1}\right)$ do código corretor de erros $\mathcal{C}$ um elemento $b_{0}+b_{1} a+\cdots+b_{n-1} a^{n-1}$ na álgebra de grupo $\mathbb{F} G$. E geralmente, ignoramos esta aplicação e pensamos em uma palavra em $\mathcal{C}$ como um elemento de $\mathbb{F} G$ ou vice-versa.

Consideremos o epimorfismo de anéis

$$
\begin{aligned}
& \Theta: \mathbb{F}[\mathrm{X}] \longrightarrow \mathbb{F} G \\
& \mathrm{f}(\mathrm{x}) \mapsto f(a)
\end{aligned}
$$

Segue, do teorema do homomorfismo para anéis, que

$$
\mathbb{F} G \cong \frac{\mathbb{F}[X]}{\operatorname{Ker} \Theta}
$$

onde $\operatorname{Ker} \Theta=\left\langle X^{n}-1\right\rangle$.

Neste isomorfismo o elemento $a \in G$ corresponde à classe de $\bar{X} \mathrm{em}$ $\frac{\mathbb{F}[X]}{\left\langle X^{n}-1\right\rangle}=\mathcal{R}_{n}$ 
Definição 3.3.1. A aplicação linear

$$
\begin{aligned}
& \sigma: \quad \mathbb{F}^{n} \quad \longrightarrow \quad \mathbb{F}^{n} \\
& \left(b_{0}, b_{1}, \ldots, b_{n-1}\right) \mapsto\left(b_{n-1}, b_{0}, \ldots, b_{n-2}\right)
\end{aligned}
$$

é chamada de troca cíclica.

Note que, $\sigma$ é um isomorfismo de $\mathbb{F}$-espaços vetoriais.

Definição 3.3.2. Um código linear $\mathcal{C} \subset \mathbb{F}^{n}$ diz-se cíclico se

$$
\left(c_{0}, c_{1}, \ldots, c_{n-1}\right) \in \mathcal{C} \quad \text { implica que }\left(c_{n-1}, c_{0}, \ldots, c_{n-2}\right) \in \mathcal{C}
$$

Equivalentemente, o código linear $\mathcal{C} \subset \mathbb{F}^{n}$ será um código cíclico se $\sigma(c) \in \mathcal{C}$ para todo $c \in \mathcal{C}$.

Observemos que, dado $c=\left(c_{0}, c_{1}, \ldots, c_{n-1}\right) \in \mathbb{F}^{n}$ temos que $\sigma(c)=$ $\left(c_{n-1}, c_{0}, \ldots, c_{n-2}\right) \mathrm{e}$

$$
\begin{aligned}
\psi(\sigma(c)) & =\psi\left(c_{n-1}, c_{1}, \ldots, c_{n-2}\right) \\
& =c_{n-1}+c_{0} a+\ldots+c_{n-2} a^{n-1} \\
& =a\left(c_{0}+c_{1} a+\ldots+c_{n-1} a^{n-1}\right) \\
& =a \psi(c) .
\end{aligned}
$$

Consequentemente, aplicar $\sigma$ num elemento $c$ de $\mathbb{F}^{n}$ se traduz em multiplicar por a sua imagem $\psi(c)$ em $\mathbb{F} G$. Como conseqüência imediata temos o seguinte teorema.

Teorema 3.3.3. Um subespaço vetorial $\mathcal{C}$ de $\mathbb{F}^{n}$ é um código cíclico se, e somente se, $\psi(\mathcal{C})$ é um ideal de $\mathbb{F} G$.

Demonstração: Obviamente, $\psi(\mathcal{C})$ é um $\mathbb{F}$-subespaço vetorial de $\mathbb{F} G$.

Seja $\alpha \in \psi(\mathcal{C})$ então existe $c \in \mathcal{C}$ tal que $\psi(c)=\alpha$. Dado $a \in G$ temos que

$$
a \alpha=a \psi(c)=\psi(\sigma(c)) \in \psi(\mathcal{C})
$$

Portanto, $\psi(\mathcal{C})$ é um ideal de $\mathbb{F} G$.

Reciprocamente, seja $c \in \mathcal{C}$ então $\psi(c) \in \psi(\mathcal{C})$ e como $\psi(\mathcal{C})$ é um ideal de $\mathbb{F} G$, temos que $a \psi(c)=\psi(\sigma(c)) \in \psi(\mathcal{C})$, isto é, $\sigma(c) \in \mathcal{C}, \forall c \in \mathcal{C}$. Portanto, $\mathcal{C}$ é um código cíclico. 
A partir de agora, assumiremos sempre que $\operatorname{mdc}(n, q)=1$.

Segue da Proposição 1.4.4, que um código $\mathcal{C}$ em $\mathbb{F}^{n}$ é cíclico se, e somente se, $\psi(\mathcal{C})$ é da forma $\psi(\mathcal{C})=\mathbb{F} G f(a)$, onde $f(X) \in \mathbb{F}[X]$ é um divisor de $X^{n}-1$.

Note que, se soubermos fatorar o polinômio $X^{n}-1$ conheceremos todos os códigos cíclicos em $\mathbb{F} G$.

A partir de agora, o polinômio $g$ denotará sempre um divisor de $X^{n}-1$ e denotaremos por $h$ o quociente $h(X)=\left(X^{n}-1\right) / g(X)$.

Faremos um abuso de notação: como $\Theta$ é um isomorfismo de $\mathcal{R}_{n}$ em $\mathbb{F} G$ nós identificaremos uma palavra $f$ em $\mathcal{C}$ com sua imagem em $\mathbb{F} G$ e vice-versa, sem fazer mensão explicita.

Se $\psi(\mathcal{C})=\mathbb{F} G g(a)$ é um código cíclico então vamos chamar o elemento $g(a)$ de gerador principal de $\mathcal{C}$. Note que há vários geradores para o código cíclico $\psi(\mathcal{C})$, mas estamos interessados neste que é proveniente da fatoração de $X^{n}-1$.

Seja $\mathcal{C}=\langle g(a)\rangle$ um código cíclico em $\mathbb{F} G$.

Seja $\alpha \in \mathcal{C}$ então $\alpha=k(a) g(a)$, para algum $k(a) \in \mathbb{F} G$. Logo, existe um único $v(X) \in \mathbb{F}[X]_{n-1}$ tal que $v(a)=k(a) g(a)$. Assim, $v(X) \equiv k(X) g(X)$ $\left(\bmod \left(X^{n}-1\right)\right)$. Como $g(X) \mid\left(X^{n}-1\right)$ temos que $v(X)=k_{1}(X) g(X)$, para algum $k_{1}(X) \in \mathbb{F}[X]$.

Assim, faremos um novo abuso de notação: quando $\alpha=k(a) g(a) \in \mathcal{C}$ escreveremos o polinômio correspondente em $\mathbb{F}[X]_{n-1}$ por $\alpha(X)=k(X) g(X)$.

Assim, se $f(a) \in \mathbb{F} G g(a)$ temos que $f(a)=\delta(a) g(a)$, para algum $\delta(a) \in$ $\mathbb{F} G$. Portanto, $f(a) h(a)=\delta(a) g(a) h(a)=0$.

Reciprocamente, suponhamos que $f(a) h(a)=0 \mathrm{em} \mathbb{F} G$. Como $g$ e $h$ são primos entre si temos que existem polinômios $r$ e $s$ tais que $r(X) g(X)+$ $s(X) h(X)=1$. Logo, $r(a) g(a)+s(a) h(a)=1$ donde $f(a) r(a) g(a)+$ $f(a) s(a) h(a)=f(a)$.

Portanto, $f(a) r(a) g(a)=f(a)$, ou seja, $f(a) \in \mathbb{F} G g(a)$.

Assim, um elemento $f(a)$ pertence a $\mathbb{F} G g(a)$ se, e somente se, $f(a) h(a)=$ 0 em $\mathbb{F} G$.

Por causa disso, o elemento $h(a)$ recebe o nome de elemento de teste.

Note que, $e=r(a) g(a)$ é a identidade em $\mathbb{F} G g(a)$ e, é um elemento idempotente de $\mathbb{F} G$.

Vamos agora encontrar matrizes de codificação $G$ e de teste $H$ do código cíclico $\mathcal{C}$. Inicialmente, temos o seguinte. 
Teorema 3.3.4. Seja $\mathcal{I}=\mathbb{F G} g(a)$, onde $g(X)=g_{0}+g_{1} X+\ldots+g_{s} X^{s}$ é um divisor de $X^{n}-1$ de grau s. Então $B=\left\{g(a), a g(a), \ldots, a^{n-s-1} g(a)\right\}$ é uma base de $\mathcal{I}$ como espaço vetorial sobre $\mathbb{F}$, a dimensão de $\mathcal{I}$ é $n-s$ e o código $\mathcal{C}=\psi^{-1}(\mathcal{I})$ tem matriz de codificação

$$
G=\left(\begin{array}{cccc}
g_{0} & 0 & \cdots & 0 \\
g_{1} & g_{0} & \cdots & 0 \\
\vdots & \vdots & \ddots & g_{0} \\
g_{s} & g_{s-1} & \cdots & g_{1} \\
0 & g_{s} & \cdots & g_{2} \\
0 & 0 & & \\
\vdots & \vdots & \ddots & \vdots \\
0 & 0 & \cdots & g_{s}
\end{array}\right) .
$$

Demonstração: Vamos mostrar que $B$ é uma base de $\mathcal{I}$ como espaço vetorial sobre $\mathbb{F}$.

É fácil ver que os elementos de $B$ são linearmente independentes.

Vamos mostrar que os elementos de $B$ geram $\mathcal{I}$ sobre $\mathbb{F}$.

De fato, se $0 \neq p(a) \in \mathcal{I}$ temos que

$$
p(a)=f(a) g(a)
$$

onde $f(X) \in \mathbb{F}[X]$.

Pelo algoritmo da divisão temos que existem $q(X), r(X) \in \mathbb{F}[X]$ tais que

$$
\begin{gathered}
f(X)=h(X) q(X)+r(X) \\
\begin{array}{c}
\operatorname{com} r(X)=r_{0}+r_{1} X+\cdots+r_{n-s-1} X^{n-s-1} . \\
\text { Logo, } \\
p(a)=h(a) q(a) g(a)+r(a) g(a) . \\
\text { Assim, } p(a)=r(a) g(a) \text {, isto é, } \\
p(a)=r_{0} g(a)+r_{1} a g(a)+\cdots+r_{n-s-1} a^{n-s-1} g(a) .
\end{array}
\end{gathered}
$$

Concluimos que $B$ é uma base de $\mathcal{I}$, logo a dimensão de $\mathcal{I}$ é $n-s$.

Sabemos que uma matriz de codificação $G$ do código $\mathcal{C}$ é qualquer matriz $n \times(n-s)$ cujas colunas formam uma base para $\mathcal{C}$.

Assim, uma matriz de codificação $G$ de $\mathcal{C}$ obtida da base acima tem a forma 


$$
G=\left(\begin{array}{cccc}
g_{0} & 0 & \cdots & 0 \\
g_{1} & g_{0} & \cdots & 0 \\
\vdots & \vdots & \ddots & g_{0} \\
g_{s} & g_{s-1} & \cdots & g_{1} \\
0 & g_{s} & \cdots & g_{2} \\
0 & 0 & & \\
\vdots & \vdots & \ddots & \vdots \\
0 & 0 & \cdots & g_{s}
\end{array}\right)
$$

Exemplo 3.3.5. Sejam $\mathbb{F}$ o corpo com dois elementos e $G=\left\{1, a, a^{2}, a^{3}, a^{4}\right.$, $\left.a^{5}, a^{6}, a^{7}, a^{8}\right\}$ um grupo cíclico de ordem 9. Considere o código cíclico binário $\mathcal{C}$ cujo gerador principal é $g(a)=a^{2}+a+1$ em $\mathbb{F} G$.

Assim, uma base para o código $\mathcal{C}$ é $B=\left\{a^{2}+a+1, a^{3}+a^{2}+a, a^{4}+a^{3}+\right.$ $\left.a^{2}, a^{5}+a^{4}+a^{3}, a^{6}+a^{5}+a^{4}, a^{7}+a^{6}+a^{5}, a^{8}+a^{7}+a^{6}\right\}$ e a dimensão de $\mathcal{C}$ é 7 . O código $\mathcal{C}$ tem matriz de codificação nesta base dada por

$$
G=\left(\begin{array}{lllllll}
1 & 0 & 0 & 0 & 0 & 0 & 0 \\
1 & 1 & 0 & 0 & 0 & 0 & 0 \\
1 & 1 & 1 & 0 & 0 & 0 & 0 \\
0 & 1 & 1 & 1 & 0 & 0 & 0 \\
0 & 0 & 1 & 1 & 1 & 0 & 0 \\
0 & 0 & 0 & 1 & 1 & 1 & 0 \\
0 & 0 & 0 & 0 & 1 & 1 & 1 \\
0 & 0 & 0 & 0 & 0 & 1 & 1 \\
0 & 0 & 0 & 0 & 0 & 0 & 1
\end{array}\right) .
$$

Considere $\alpha \in \mathbb{F} G$, onde $\alpha=\sum_{i=0}^{m} \alpha_{i} a^{i}$, onde $m \leq n-1$.

Defino o elemento recíproco de $\alpha$ como

$$
\alpha^{*}=a^{m} \sum_{i=0}^{m} \alpha_{i} a^{-i} .
$$

Note que, $\alpha^{*} \in \mathbb{F} G$.

Seja $f(X)=\alpha_{0}+\alpha_{1} X+\cdots+\alpha_{m} X^{m} \in \mathbb{F}[X]$ entào $f(a)=\alpha$.

Defino o polinômio recíproco de $f(X)$ como sendo a função racional

$$
f^{*}(X)=X^{n} f\left(X^{-1}\right) \text {. }
$$


50 CAPÍTULO 3. CONCEITOS BÁSICOS DA TEORIA DE CÓDIGOS

Note que,

$$
\begin{aligned}
f^{*}(X) & =X^{m} f\left(X^{-1}\right)=X^{m}\left(\alpha_{0}+\alpha_{1} X^{-1}+\cdots+\alpha_{m} X^{-m}\right) \\
& =\alpha_{0} X^{m}+\alpha_{1} X^{m-1}+\cdots+\alpha_{m} \in \mathbb{F}[X]
\end{aligned}
$$

e $f^{*}(a)=\alpha^{*}$

Proposição 3.3.6. Sejam $\mathbb{F}$ um corpo e $f(X) \in \mathbb{F}[X]$ de grau n. Sejam $f(X), g(X) \in \mathbb{F}[X]$ tais que $g(X)$ divide $f(X)$. Então $g^{*}(X)$ divide $f^{*}(X)$. Se $g(X)$ divide $X^{n}-1$ então $g^{*}(X)$ também divide $X^{n}-1$.

Demonstração: Se $g(X) \mid f(X)$ temos que $f(X)=k(X) g(X)$, onde $k(X) \in \mathbb{F}[X]$.

Temos que $\operatorname{grau}(g(X)) \leq \operatorname{grau}(f(X))$. Suponhamos que grau $(g(X))=$ $s<n$.

Temos

$$
\begin{aligned}
f^{*}(X)=X^{n} f\left(X^{-1}\right) & =X^{n}\left[k\left(X^{-1}\right) g\left(X^{-1}\right)\right] \\
& =X^{n-s} k\left(X^{-1}\right) X^{s} g\left(X^{-1}\right) \\
& =X^{n-s}\left(k\left(X^{-1}\right)\right) g^{*}(X) \\
& =k^{*}(X) g^{*}(X)
\end{aligned}
$$

portanto, $g^{*}(X) \mid f^{*}(X)$.

Note que, $\left(X^{n}-1\right)^{*}=X^{n}\left(X^{-n}-1\right)=1-X^{n}=-\left(X^{n}-1\right)$.

Logo, $-\left(X^{n}-1\right)^{*}=X^{n}-1$.

Se $g(X) \mid\left(X^{n}-1\right)$ então segue, da primeira parte, que $g^{*}(X) \mid\left(X^{n}-1\right)^{*}$.

Então, $g^{*}(X) \mid\left(X^{n}-1\right)$.

Lema 3.3.7. Sejam $b=\left(b_{0}, \ldots, b_{n-1}\right)$ e $c=\left(c_{0}, \ldots, c_{n-1}\right)$. Então $b(a) c(a)=$ 0 em $\mathbb{F} G$ se, e somente se, $b^{*}=\left(b_{n-1}, \ldots, b_{0}\right)$ é ortogonal ao vetor c e a toda troca cíclica deste vetor em $\mathbb{F}^{n}$.

Demonstração: Sejam $b(a)=\sum_{i=0}^{n-1} b_{i} a^{i}$ e $c(a)=\sum_{j=0}^{n-1} c_{j} a^{j}$.

Então

$$
b(a) c(a)=\sum_{i, j}\left(b_{i} c_{j}\right)\left(a^{i} a^{j}\right) .
$$

Podemos escrever este produto como

$$
b(a) c(a)=\sum_{k=0}^{n-1} d d_{k} a^{k}
$$


onde

$$
d_{k}=\sum_{i+j=k} b_{i} c_{j} .
$$

Então, $b(a) c(a)=0$ em $\mathbb{F} G$ se, e somente se, $d_{k}=0$, ou seja,

$$
\begin{aligned}
& b_{n-1} c_{1}+b_{n-2} c_{2}+b_{n-3} c_{3}+\cdots+b_{0} c_{0}=0 \\
& b_{n-1} c_{2}+b_{n-2} c_{3}+b_{n-3} c_{4}+\cdots+b_{0} c_{1}=0 \\
& b_{n-1} c_{3}+b_{n-2} c_{4}+b_{n-3} c_{5}+\cdots+b_{0} c_{2}=0 \\
& \begin{array}{llllllll}
\cdots & \ldots & \ldots & \ldots & \ldots & \ldots & \ldots & =0
\end{array} \\
& b_{n-1} c_{0}+b_{n-2} c_{1}+b_{n-3} c_{2}+\cdots+b_{0} c_{n-1}=0
\end{aligned}
$$

o que equivale a

$$
\begin{aligned}
& \left(b_{n-1}, b_{n-2}, b_{n-3}, \ldots, b_{0}\right) \cdot\left(c_{1}, c_{2}, c_{3}, \ldots, c_{0}\right)=b^{*} \cdot \sigma^{n-1}(c)=0 \\
& \left(b_{n-1}, b_{n-2}, b_{n-3}, \ldots, b_{0}\right) \cdot\left(c_{2}, c_{3}, c_{4}, \ldots, c_{1}\right)=b^{*} \cdot \sigma^{n-2}(c)=0 \\
& \left(b_{n-1}, b_{n-2}, b_{n-3}, \ldots, b_{0}\right) \cdot\left(c_{3}, c_{4}, c_{5}, \ldots, c_{2}\right)=b^{*} \cdot \sigma^{n-3}(c)=0 \\
& \begin{array}{llllllllll}
\cdots & \cdots & \cdots & \cdots & \cdots & \cdots & \cdots & \cdots & \cdots & \cdots
\end{array} \\
& \left(b_{n-1}, b_{n-2}, b_{n-3}, \ldots, b_{0}\right) \cdot\left(c_{0}, c_{1}, c_{2}, \ldots, c_{n-1}\right)=b^{*} \cdot c \quad=0
\end{aligned}
$$

Portanto, $b^{*}$ é ortogonal ao vetor $c$ e a toda troca cíclica deste vetor em $\mathbb{F}^{n}$.

Teorema 3.3.8. Seja $\mathcal{C}$ um código cíclico, onde $\mathcal{I}=\psi(\mathcal{C})=\langle g(a)\rangle$, com $g(X)$ um divisor de $X^{n}-1$ de grau s. Se $X^{n}-1=g(X) h(X) \operatorname{com} h(X)=$ $h_{0}+h_{1} X+\cdots+h_{n-s} X^{n-s}$ então uma matriz de teste de $\mathcal{C}$ é dada por

$$
H=\left(\begin{array}{cccccccc}
h_{n-s} & h_{n-s-1} & \cdots & \cdots & h_{0} & 0 & \cdots & 0 \\
0 & h_{n-s} & \cdots & \cdots & h_{1} & h_{0} & \cdots & 0 \\
\vdots & \vdots & \ddots & \vdots & \vdots & \vdots & \ddots & \vdots \\
0 & 0 & \cdots & h_{n-s} & h_{n-s-1} & \cdots & & h_{0}
\end{array}\right)
$$

Demonstração: Note que $H$ é uma matriz de teste de $\mathcal{C}$ se, e somente se, $H G=0$.

Observemos que cada coeficiente do produto $H G$ se obtém multiplicando o vetor $\left(h_{n-1}, h_{n-2}, \ldots, h_{0}\right)$ por uma troca cíclica do vetor $\left(g_{0}, g_{1}, \ldots, g_{n-1}\right)$.

Como $h(a) g(a)=0 \mathrm{em} \mathbb{F} G$ o Lema 3.3.7 mostra diretamente que o procluto $H G=0$. 
Corolário 3.3.9. Seja $\mathcal{C}$ um código cíclico, onde $\mathcal{I}=\psi(\mathcal{C})=\langle g(a)\rangle$, com $g(X)$ um divisor de $X^{n}-1$ de grau s. Então $\mathcal{C}^{\perp}$ é cíclico e $\mathcal{C}^{\perp}=\dot{\psi}^{-1}(\mathcal{J})$, onde $\mathcal{J}=\left\langle h^{*}(a)\right\rangle$.

Demonstração: Observe que a dimensão de $\mathcal{C}$ é $n-s$ e portanto a dimensão de $\mathcal{C}^{\perp}$ é $s$.

Seja $H$ uma matriz de teste de $\mathcal{C}$, como no teorema anterior.

Como $H G=0$ o espaço gerado pelas linhas de $H$ está contido em $\mathcal{C}^{\perp} \mathrm{e}$ como o posto de $H$ é igual a $s$ estes dois subespaços coincidem. Portanto, a matriz de teste $H$ é uma matriz geradora de $\mathcal{C}^{\perp}$.

Como $X^{n}-1=g(X) h(X)$ então $h(X)$ divide $X^{n}-1$ e segue da Proposição 3.3.6 que $h^{*}(X)$ divide $X^{n}-1$. Portanto, $h^{*}(a)$ é o gerador principal do código cíclico $\mathcal{C}^{\perp}$.

Exemplo 3.3.10. Utilizando o código do Exemplo 3.3.5 vamos determinar uma matriz de teste $H$ deste código.

Temos que $h(X)=\left(X^{9}-1\right) /\left(X^{2}+X+1\right)=X^{7}+X^{6}+X^{4}+X^{3}+X+1$. Então o código cíclico $\mathcal{C}$ tem matriz de teste

$$
H=\left(\begin{array}{lllllllll}
1 & 1 & 0 & 1 & 1 & 0 & 1 & 1 & 0 \\
0 & 1 & 1 & 0 & 1 & 1 & 0 & 1 & 1
\end{array}\right)
$$

\subsection{Zeros do código cíclico}

Uma outra caracterização dos códigos cíclicos é feita através das raízes do polinômio $X^{n}-1$.

Seja $X^{n}-1=\prod_{i} m_{i}(X)$ a fatoração de $X^{n}-1$ em fatores mônicos irredutíveis sobre $\mathbb{F}$.

Se $\xi$ é uma raiz de $m_{i}(X)$ em alguma extensão de $\mathbb{F}$ então $m_{i}(X)$ é o polinômio minimal de $\xi$ sobre $\mathbb{F}$.

Considere o código cíclico $\mathcal{C}$ gerado por $m_{i}(X)$.

Seja $f(X) \in \mathbb{F}[X]$. Então $f(a) \in \mathcal{C}$ se, e somente se, $f(a)=k(a) m_{i}(a)$, para algum $k(a) \in \mathbb{F} G$; isto é, se e somente se $f(X)=k(X) m_{i}(X)$ e, neste caso, $f(\xi)=k(\xi) m_{i}(\xi)=0$.

Reciprocamente, se $f(\xi)=0$ então $m_{i}(X) \mid f(X)$, donde $f \in \mathcal{C}$.

Resumindo, $f \in \mathcal{C}$ se e somente se $f(\xi)=0$.

Mas geralmente. temos o seguinte. 
Teorema 3.4.1. Seja $g(X)$ um divisor de $X^{n}-1$ e seja $\left\{\xi_{1}, \xi_{2}, \ldots, \xi_{u}\right\} o$ conjunto das raizes de $g(X)$ num corpo de decomposição de $X^{n}-1$ sobre $\mathbb{F}$. Então

$$
\psi(\mathcal{C})=\langle g(a)\rangle=\left\{f(a) \in \mathbb{F} G \mid f(X) \in \mathbb{F}[X], f\left(\xi_{1}\right)=0, \ldots, f\left(\xi_{u}\right)=0\right\} .
$$

Demonstração: É claro que, se $f(a) \in\langle g(a)\rangle$ então $f(a)=k(a) g(a)$, para algum $k(a) \in \mathbb{F} G$. Logo, $f\left(\xi_{1}\right)=0, \ldots, f\left(\xi_{u}\right)=0$.

Reciprocamente, temos que duas raízes diferentes de $g(X)$ podem ter o mesmo polinômio minimal.

Sejam $m_{1}(X), m_{2}(X), \ldots, m_{t}(X)$ os polinômios minimais das raízes de $g(X)$, diferentes dois a dois. Então $g(X)=m_{1}(X) m_{2}(X) \cdots m_{t}(X)$.

Como todas as raízes de $g(X)$ são raízes de $f(X)$ temos que $m_{i}(X) \mid f(X)$, $1 \leq i \leq t$ e, como eles são relativamente primos

$$
\left(m_{1}(X) m_{2}(X) \cdots m_{t}(X)\right) \mid f(X)
$$

isto é, $g(X) \mid f(X)$.

Portanto, $f(a) \in\langle g(a)\rangle$.

Dizemos que $\xi$ é um zero do código cíclico $\mathcal{C}$. As outras raízes $n$-ésimas da unidade que não são zeros do código $\mathcal{C}$ dizem-se os não zeros do código e estas são as raízes do polinômio $h=\left(X^{n}-1\right) / g$.

Note que, se $\left\{\xi_{1}, \ldots, \xi_{u}\right\}$ é qualquer conjunto de raízes de $X^{n}-1$ então o polinômio gerador do código cíclico

$$
\left\{f(a) \in \mathbb{F} G \mid f(X) \in \mathbb{F}[X], f\left(\xi_{1}\right)=0, \ldots, f\left(\xi_{u}\right)=0\right\}
$$

é o mínimo multiplo comum dos polinômios minimais para as raízes $\xi_{1}, \ldots, \xi_{u}$.

Com essa caracterização dos códigos cíclicos podemos determinar rapidamente uma matriz de teste $H$.

Seja $\left\{\xi_{1}, \ldots, \xi_{u}\right\}$ um conjunto de raízes de $X^{n}-1$ num corpo de decomposição $\mathbb{K}$ do polinômio $X^{n}-1$ e seja $[\mathbb{K}: \mathbb{F}]=d$.

Seja $f(X)=\sum_{j=0}^{n-1} b_{j} X^{j} \in \mathbb{F}[X]$.

Logo, $f \in \mathcal{C}$ se, e somente se,

$$
f\left(\xi_{i}\right)=\sum_{j=0}^{n-1} b_{j} \xi_{i}^{j}=0, \quad \text { para } i=1, \ldots, u
$$


Podemos descrever o código cíclico $\mathcal{C}$ definido pelo elemento $g(a)$, onde $g(X)=\left(X-\xi_{1}\right) \cdots\left(X-\xi_{u}\right)$ como sendo o conjunto dos elementos $b=$ $\left(b_{0}, \ldots, b_{n-1}\right) \in \mathbb{F}^{n}$ tais que

$$
b_{0}+b_{1} \xi_{i}+b_{2} \xi_{i}^{2}+\cdots+b_{n-1} \xi_{i}^{n-1}=0
$$

para $i=1, \ldots, u$, ou seja, o conjunto dos elementos $b=\left(b_{0}, \ldots, b_{n-1}\right) \in \mathbb{F}^{n}$ tais que

$$
H_{1} b=0
$$

onde $H_{1}$ é a seguinte matriz com entradas em $\mathbb{K}$

$$
H_{1}=\left(\begin{array}{ccccc}
\xi_{1}^{0} & \xi_{1}^{1} & \xi_{1}^{2} & \cdots & \xi_{1}^{n-1} \\
\xi_{2}^{0} & \xi_{2}^{1} & \xi_{2}^{2} & \cdots & \xi_{2}^{n-1} \\
\vdots & \vdots & \vdots & \ddots & \vdots \\
\xi_{u}^{0} & \xi_{u}^{1} & \xi_{u}^{2} & \cdots & \xi_{u}^{n-1}
\end{array}\right)
$$

Como $\mathbb{K}$ pode ser visto como espaço vetorial sobre $\mathbb{F}$ de dimensão finita $d$, então podemos representar os elementos $\xi_{i}^{j} \in \mathbb{K}$ como combinação linear de uma base ordenada de $\mathbb{K}$ de dimensão $d$, isto é,

$$
\xi_{i}^{j}=\sum_{k=0}^{d-1} \lambda_{k} \gamma^{k}
$$

onde $i=1, \ldots, u, j=0, \ldots, n-1, \lambda_{k} \in \mathbb{F}$ e $B=\left\{1, \gamma, \ldots, \gamma^{d-1}\right\}$ é uma base de $\mathbb{K}$.

Escrevemos $\left[\xi_{i}^{j}\right]$ o vetor coluna de $\mathbb{F}^{d}$ das coordenadas de $\xi_{i}^{j}$ com relação à base $B$.

Definimos a matriz $\mathrm{H}_{2}$ como sendo

e temos que

$$
H_{2}=\left(\begin{array}{ccccc}
{\left[\xi_{1}^{0}\right]} & {\left[\xi_{1}^{1}\right]} & {\left[\xi_{1}^{2}\right]} & \cdots & {\left[\xi_{1}^{n-1}\right]} \\
{\left[\xi_{2}^{0}\right]} & {\left[\xi_{2}^{1}\right]} & {\left[\xi_{2}^{2}\right]} & \cdots & {\left[\xi_{2}^{n-1}\right]} \\
\vdots & \vdots & \vdots & \ddots & \vdots \\
{\left[\xi_{u}^{0}\right]} & {\left[\xi_{u}^{1}\right]} & {\left[\xi_{u}^{2}\right]} & \cdots & {\left[\xi_{u}^{n-1}\right]}
\end{array}\right)
$$

$$
b \in \mathcal{C} \Longleftrightarrow H_{2} b=0 .
$$

Como as linhas de $\mathrm{H}_{2}$ não são, necessariamente, linearmente independentes, escolhemos um conjunto maximal de linhas linearmente independentes, obtemos então uma matriz $H$ que ainda verifica

$$
b \in \mathcal{C} \quad \Longleftrightarrow \quad H b=0 .
$$


Como esta é uma matriz de posto máximo com esta propriedade, temos o seguinte.

Teorema 3.4.2. A matriz $H$ construida acima é uma matriz de teste para $\mathcal{C}$.

Exemplo 3.4.3. Sejam $\mathbb{F}$ o corpo finito com clois elementos e $G$ o grupo cíclico de ordem 7. Segue, do Exemplo 1.1.20, que se $\zeta$ é uma raiz 7-ésima primitiva da unidade sobre $\mathbb{F}$ é também um elemento primitivo de $\mathbb{F}_{8}$.

Temos que os polinômios minimais de $\zeta^{k}$ são:

$$
\begin{aligned}
& m_{0}(X)=X-1 ; \\
& m_{\zeta}(X)=m_{\zeta^{2}}(X)=m_{\zeta^{4}}(X)=(X-\zeta)\left(X-\zeta^{2}\right)\left(X-\zeta^{4}\right)=X^{3}+X+1 \\
& m_{\zeta^{3}}(X)=m_{\zeta^{6}}(X)=m_{\zeta^{5}}(X)=\left(X-\zeta^{3}\right)\left(X-\zeta^{6}\right)\left(X-\zeta^{5}\right)=X^{3}+X^{2}+1 .
\end{aligned}
$$

Defino $\mathcal{C}$ como o código cíclico gerado por $g(a)=m_{\zeta}(a) m_{\zeta^{3}}(a)$.

Seja $f(X) \in \mathbb{F}[X]$. Temos que $f(a) \in \mathcal{C}$ se, e somente se, $f(\zeta)=f\left(\zeta^{3}\right)=0$.

Portanto,

$$
H_{1}=\left(\begin{array}{ccccccc}
\zeta^{0} & \zeta^{1} & \zeta^{2} & \zeta^{3} & \zeta^{4} & \zeta^{5} & \zeta^{6} \\
\zeta^{0} & \zeta^{3} & \zeta^{6} & \zeta^{9} & \zeta^{12} & \zeta^{15} & \zeta^{18}
\end{array}\right)
$$

Escrevendo cada uma destas entradas em função da base, obtemos

$$
H_{2}=H=\left(\begin{array}{lllllll}
0 & 0 & 1 & 0 & 1 & 1 & 1 \\
0 & 1 & 0 & 1 & 1 & 1 & 0 \\
1 & 0 & 0 & 1 & 0 & 1 & 1 \\
\hline 0 & 0 & 1 & 1 & 1 & 0 & 1 \\
0 & 1 & 0 & 0 & 1 & 1 & 1 \\
1 & 1 & 1 & 0 & 1 & 0 & 0
\end{array}\right)
$$


56 CAPÍTULO 3. CONCEITOS BÁSICOS DA TEORIA DE CÓDIGOS 


\section{Capítulo 4}

\section{Códigos BCH e Reed-Solomon}

Iremos introduzir uma importante classe dos códigos conhecida como códigos BCH. Estes foram descobertos por A. Hocquenghem(1959) e independentemente por R. C. Bose e D. K. Ray-Chaudhuri (1960). A aplicação original dos códigos $\mathrm{BCH}$ foi restrita aos códigos binários de comprimento $2^{m}-1$, para algum inteiro $m$. Estes foram estendidos mais tarde por Gorenstein e Zieler (1961) para códigos não binários com elementos num corpo de Galois. O primeiro algoritmo de decodificação para códigos $\mathrm{BCH}$ binários foi desenvolvido por Peterson em 1960. Desde então, o algoritmo de Peterson foi aprimorado por Berlekamp, Massey, Chien, Forney e muitos outros.

Estes códigos formam uma importante classe de códigos cíclicos por várias razões, entre elas, para qualquer inteiro positivo $d$, podemos construir um código $\mathrm{BCH}$ de distância mínima maior ou igual a $d$, além deles terem boas propriedades detectoras e corretoras de erros. Quando seu comprimento não for muito longo a codificação e decodificação é relativamente simples.

\subsection{Códigos Bose-Chaudhuri-Hocquenghem - $\mathrm{BCH}$}

Consideremos $\mathbb{F}$ um corpo finito com $q$ elementos e seja $m$ a ordem multiplicativa de $q$ módulo $n$.

Denotaremos por $\mathbb{F}_{q^{m}}$ o corpo finito com $q^{m}$ elementos.

Sejam $b$ e $\delta$ inteiros positivos, e $\zeta \in \mathbb{F}_{q^{m}}$ uma raiz $n$-ésima primitiva da unidade. 
Definição 4.1.1. Chama-se código BCH de distância designada $\delta$, $2 \leq \delta \leq n$, ao código cíclico de comprimento $n$ sobre $\mathbb{F}$ cujo gerador principal $g(X)$ é o mínimo multiplo comum dos polinômios mínimais de $\zeta^{b}, \zeta^{b+1}, \ldots, \zeta^{b+\delta-2}$.

Geralmente tomamos $b=1$ e, neste caso, dizemos que é um código $\mathrm{BCH}$ em sentido estrito.

Lembremos que, se $n=q^{m}-1$ então $\zeta$ é um elemento primitivo de $\mathcal{F}_{q^{m}}$.

Definição 4.1.2. Se $n=q^{m}-1$ dizemos que o código $B C H$ é primitivo.

Exemplo 4.1.3. Sejam $q=2$ e $n=15$. O corpo de decomposição do polinômio $X^{15}-1$ sobre $\mathbb{F}$ é o corpo finito $\mathbb{F}_{2^{4}}$ com 16 elementos.

Vamos encontrar o código $\mathrm{BCH}$ em sentido estrito, primitivo, com distância designada $\delta=3$.

Começaremos encontrando as raízes 15-ésima primitiva da unidade sobre $\mathbb{F}$.

O polinômio $m(X)=X^{4}+X+1$ é irredutível sobre $\mathbb{F}$.

Para verificar isto, mostraremos que $m(X)$ não pode ser escrito como produtos de fatores lineares e nem produtos de fatores quadráticos.

De fato, como $m(0)=1$ e $m(1)=1$ o polinômio $m(X)$ não tem fatores lineares.

Vamos verificar que $m(X)$ não tem fatores quadrátricos, suponhamos que

$$
\begin{aligned}
X^{4}+X+1 & =\left(X^{2}+a X+b\right)\left(X^{2}+c X+d\right) \\
& =X^{4}+(c+a) X^{3}+(d+b+a c) X^{2}+(a d+b c) X+b d
\end{aligned}
$$

onde $a, b, c$ e $d \in \mathbb{F}$.

Portanto,

$$
\begin{aligned}
c+a & =0 \\
d+b+a c & =0 \\
a d+b c & =1 \\
b d & =1
\end{aligned}
$$

logo, cle (4.4) vem que $b=d=1$ e em (4.3) obtemos $a+c=1$, o que contradiz (4.1).

Assim $m(X)$ também não pode ser escrito como produto de polinômios de grau dois. Portanto, $m(X)$ ć irrechutível sobre $\mathbb{F}$.

Como $q=2$ e $\operatorname{grau}(m(X))=4$ temos

$$
\mathbb{F}_{2^{4}}=\frac{\mathbb{F}[X]}{\left\langle X^{4}+X+1\right\rangle}=\left\{\overline{\lambda_{0}+\lambda_{1} X+\lambda_{2} X^{2}+\lambda_{3} X^{3}} \mid \lambda_{0}, \lambda_{1}, \lambda_{2}, \lambda_{3} \in \mathbb{F}\right\} .
$$


Vamos descrever os elementos de $\mathbb{F}_{2^{4}}$.

Seja $\zeta$ uma raiz do polinômio $m(X)$ então poclemos representar $\mathbb{F}_{2^{4}}$ como o conjunto dos polinômios em $\zeta$ de grau menor ou igual a 3 , com a condição $\zeta^{4}=\zeta+1$.

Por outro lado,

$$
\begin{aligned}
\zeta^{15} & =\left(\zeta^{5}\right)^{3} \\
& =\left(\zeta^{4} \zeta\right)^{3} \\
& =(\zeta(\zeta+1))^{3} \\
& =\zeta^{3}\left(\zeta^{3}+\zeta^{2}+\zeta+1\right) \\
& =\zeta^{6}+\zeta^{5}+\zeta^{4}+\zeta^{3} \\
& =\zeta^{3}+\zeta^{2}+\zeta^{2}+\zeta+\zeta+1+\zeta^{3} \\
& =1
\end{aligned}
$$

Logo, a ordem de $\zeta$ deve dividir 15. Mas $\zeta^{3} \neq 1$ e $\zeta^{5}=\zeta \zeta^{4}=\zeta^{2}+\zeta \neq 1$ pois, caso contrário, $\zeta$ seria raiz do polinômio $X^{2}+X+1$, um absurdo. Portanto, $\zeta$ tem ordem 15 que significa que $\zeta$ é um gerador do grupo cíclico $\mathbb{F}_{16}^{*}$.

Assim, todo elemento, não nulo, de $\mathbb{F}_{2^{4}}$ tem a forma $\zeta^{j}$, para $j=0,1, \cdots, 14$.

Vamos dispor todos os elementos de $\mathbb{F}_{2^{4}}$ em uma tabela.

Usando o fato que $\zeta^{4}=\zeta+1$ temos

\begin{tabular}{|c||l|}
\hline expoente de $\zeta$ & polinômio \\
\hline 0 & 1 \\
\hline 1 & $\zeta$ \\
\hline 2 & $\zeta^{2}$ \\
\hline 3 & $\zeta^{3}$ \\
\hline 4 & $\zeta+1$ \\
\hline 5 & $\zeta^{2}+\zeta$ \\
\hline 6 & $\zeta^{3}+\zeta^{2}$ \\
\hline 7 & $\zeta^{3}+\zeta+1$ \\
\hline 8 & $\zeta^{2}+1$ \\
\hline 9 & $\zeta^{3}+\zeta$ \\
\hline 10 & $\zeta^{2}+\zeta+1$ \\
\hline 11 & $\zeta^{3}+\zeta^{2}+\zeta$ \\
\hline 12 & $\zeta^{3}+\zeta^{2}+\zeta+1$ \\
\hline 13 & $\zeta^{3}+\zeta^{2}+1$ \\
\hline 14 & $\zeta^{3}+1$ \\
\hline
\end{tabular}

Note que $\zeta$ é uma raiz 15-ésima primitiva da uniclade sobre $\mathbb{F}$, donde toda raiz de $m(X)$ é uma raiz 15-ésima primitiva da unidade.

Como $m(X)$ é irredutível sobre $\mathbb{F}$. cle é o polinòmio minimal de $\zeta$ sobre $\mathbb{F}$. 
A classe ciclotômica de 1 módulo 15 é $\{1,2,4,8\}$. Então, pelo Teorema 1.1.19, o polinômio minimal de $\zeta$ e $\zeta^{2}$ sobre $\mathbb{F}$ é

$$
m_{\zeta}(X)=m_{\zeta^{2}}(X)=(X-\zeta)\left(X-\zeta^{2}\right)\left(X-\zeta^{4}\right)\left(X-\zeta^{8}\right) .
$$

Assim, o polinômio gerador deste código $\mathrm{BCH}$ é

$$
g(X)=m m c\left\{m_{\zeta}(X), m_{\zeta^{2}}(X)\right\}=X^{4}+X+1 .
$$

Considere agora a matriz

$$
[H]=\left(\begin{array}{lllllllllllllll}
1 & \zeta & \zeta^{2} & \zeta^{3} & \zeta^{4} & \zeta^{5} & \zeta^{6} & \zeta^{7} & \zeta^{8} & \zeta^{9} & \zeta^{10} & \zeta^{11} & \zeta^{12} & \zeta^{13} & \zeta^{14}
\end{array}\right)
$$

onde cada entrada é interpretada como um vetor coluna de $\mathbb{F}^{4}$ das coordenadas de $\zeta^{j}$ com relação à base $\left\{1, \zeta, \zeta^{2}, \zeta^{3}\right\}$, onde $j=0, \cdots, 14$.

Logo, pelo Teorema 3.4 .2 , temos que, a matriz de teste é

$$
H=\left(\begin{array}{lllllllllllllll}
1 & 0 & 0 & 0 & 1 & 0 & 0 & 1 & 1 & 0 & 1 & 0 & 1 & 1 & 1 \\
0 & 1 & 0 & 0 & 1 & 1 & 0 & 1 & 0 & 1 & 1 & 1 & 1 & 0 & 0 \\
0 & 0 & 1 & 0 & 0 & 1 & 1 & 0 & 1 & 0 & 1 & 1 & 1 & 1 & 0 \\
0 & 0 & 0 & 1 & 0 & 0 & 1 & 1 & 0 & 1 & 0 & 1 & 1 & 1 & 1
\end{array}\right) .
$$

Teorema 4.1.4 (Cota de $\mathrm{BCH}$ ). A distância mínima de um código $B C H$ com distância designada $\delta$ é pelo menos $\delta$.

Demonstração: Seja $\mathcal{C}$ um código BCH com distância designada $\delta$. Segue da definição, que $\mathcal{C}$ é gerado pelo polinômio $g(X)$ que é o mínimo multiplo comum dos polinômios minimais de $\zeta^{b}, \zeta^{b+1}, \ldots, \zeta^{b+\delta-2}$, onde $b$ é um inteiro positivo e $\zeta$ é uma raiz $n$-ésima primitiva da unidade sobre $\mathbb{F}$.

Definimos a matriz $H$ de ordem $(\delta-1) \times n$ por

$$
H=\left(\begin{array}{ccccc}
\zeta^{0} & \zeta^{b} & \zeta^{2 b} & \cdots & \zeta^{(n-1) b} \\
\zeta^{0} & \zeta^{b+1} & \zeta^{2(b+1)} & \cdots & \zeta^{(n-1)(b+1)} \\
\vdots & \vdots & \vdots & \ddots & \vdots \\
\zeta^{0} & \zeta^{b+\delta-2} & \zeta^{2(b+\delta-2)} & \cdots & \zeta^{(n-1)(b+\delta-2)}
\end{array}\right) .
$$

Considere as $\delta-1$ colunas de $H$ iniciadas com $\zeta^{i_{1} b}, \zeta^{i_{2} b}, \cdots, \zeta^{i_{\delta-1} b}$, onde $0 \leq i_{1}<i_{2}<\cdots<i_{\delta-1} \leq n-1$. Estas formam uma matriz que possui o seguinte bloco principal

$$
H_{1}=\left(\begin{array}{cccc}
\zeta_{1}^{i_{1} b} & \zeta^{i_{2} b} & \cdots & \zeta^{i_{S-1} b} \\
\zeta^{i_{1}(b+1)} & \zeta^{i_{2}(b+1)} & \cdots & \zeta^{i_{S-1}(b+1)} \\
\vdots & \vdots & \ddots & \vdots \\
\zeta^{i_{1}(b+\delta-2)} & \zeta^{i_{2}(b+j-i-2)} & \cdots & \zeta^{i_{j-1}(b+j-2)}
\end{array}\right) .
$$


Como o determinante de $H_{1}$ é igual a

$$
\zeta^{\left(i_{1}+i_{2}+\cdots+i_{\delta-1}\right) b} \operatorname{det}\left(\begin{array}{cccc}
1 & 1 & \cdots & 1 \\
\zeta^{i_{1}} & \zeta^{i_{2}} & \cdots & \zeta^{i_{\delta-1}} \\
\vdots & \vdots & \ddots & \vdots \\
\zeta^{i_{1}(\delta-2)} & \zeta^{i_{2}(\delta-2)} & \cdots & \zeta^{i_{\delta-1}(\delta-2)}
\end{array}\right)
$$

Temos que $\operatorname{det} H_{1} \neq 0$, pois o determinante acima é o determinante de Vandermonde, $\operatorname{com} \zeta^{i_{r}} \neq \zeta^{i_{s}}$ se $r \neq s$, e por ser $\zeta$ uma raiz $n$-ésima primitiva da unidade.

Portanto, como os inteiros $i_{1}, i_{2}, \cdots, i_{\delta-1}$ são arbitrários, quaisquer $\delta-1$ colunas de $H$ são linearmente independentes e assim uma palavra $0 \neq c \in \mathcal{C}$ tem peso maior a $\delta-1$. Isto é, $\omega(\mathcal{C}) \geq \delta$.

Exemplo 4.1.5. Sejam $q=2$ e $n=15$. O corpo de decomposição do polinômio $X^{15}-1$ sobre $\mathbb{F}$ é $\mathbb{F}_{2^{4}}$ o corpo finito com 16 elementos.

Vamos encontrar o código $\mathrm{BCH}$ em sentido estrito, primitivo, com distância designada $\delta=4$.

Seja $\zeta$ uma raiz 15-ésima primitiva da unidade sobre $\mathbb{F}$ com polinômio minimal $m_{\zeta}(X)=X^{4}+X+1$, como no Exemplo 4.1.3.

Precisamos encontrar os polinômios minimais de $\zeta, \zeta^{2}$ e $\zeta^{3}$.

Os conjugados de $\zeta$ são:

$$
\zeta, \quad \zeta^{2}, \quad \zeta^{4}, \quad \zeta^{8}
$$

portanto, o polinômio minimal de $\zeta$ é dado por

$$
m_{\zeta}(X)=X^{4}+X+1
$$

Os conjugados de $\zeta^{3}$ são:

$$
\zeta^{3}, \quad \zeta^{6}, \quad \zeta^{12}, \quad \zeta^{9}
$$

portanto, o polinômio minimal de $\zeta^{3}$ é dado por

$$
m_{\zeta^{3}}(X)=\left(X-\zeta^{3}\right)\left(X-\zeta^{6}\right)\left(X-\zeta^{12}\right)\left(X-\zeta^{-9}\right)
$$

e utilizando a tabela construida no Exemplo 4.1 .3 temos que

$$
m_{\zeta^{3}}(X)=X^{4}+X^{3}+X^{2}+X+1 .
$$

Assim. o polinômio gerador cleste código $\mathrm{BCH}$ é 


$$
g(X)=\left(X^{4}+X+1\right)\left(X^{4}+X^{3}+X^{2}+X+1\right)
$$

e sua dimensão é $15-\operatorname{grau}(g(X))=15-8=7$.

Exemplo 4.1.6. Sejam $q=2$ e $n=31$. Vamos determinar os códigos BCH em sentido estrito, primitivo, com várias distâncias designadas.

Primeiro, calculamos as classes ciclotômicas módulo 31:

$$
\begin{aligned}
& \Omega_{0}=\{0\} \\
& \Omega_{1}=\{1,2,4,8,16\} ; \\
& \Omega_{3}=\{3,6,12,24,17\} \\
& \Omega_{5}=\{5,10,20,9,18\} \\
& \Omega_{7}=\{7,14,28,25,19\} ; \\
& \Omega_{11}=\{11,22,13,26,21\} ; \\
& \Omega_{15}=\{15,30,29,27,23\} .
\end{aligned}
$$

Podemos encontrar os polinômios minimais de todas as potências de $\zeta$, onde $\zeta$ é una raiz 31-ésima primitiva da unidade sobre $\mathbb{F}$.

Portanto,

$$
\begin{aligned}
& m_{0}(X)=X+1 ; \\
& m_{\zeta^{1}}(X)=m_{\zeta^{2}}(X)=m_{\zeta^{4}}(X)=m_{\zeta^{8}}(X)=m_{\zeta^{16}}(X)=X^{5}+X^{2}+1 \\
& m_{\zeta^{3}}(X)=m_{\zeta^{6}}(X)=m_{\zeta^{12}}(X)=m_{\zeta^{24}}(X)=m_{\zeta^{17}}(X)=X^{5}+X^{4}+X^{3}+X^{2}+1 \\
& m_{\zeta^{5}}(X)=m_{\zeta^{10}}(X)=m_{\zeta^{20}}(X)=m_{\zeta^{9}}(X)=m_{\zeta^{13}}(X)=X^{5}+X^{4}+X^{2}+X+1 ; \\
& m_{\zeta^{7}}(X)=m_{\zeta^{14}}(X)=m_{\zeta^{28}}(X)=m_{\zeta^{25}}(X)=m_{\zeta^{19}}(X)=X^{5}+X^{3}+X^{2}+X+1 ; \\
& m_{\zeta^{11}}(X)=m_{\zeta^{22}}(X)=m_{\zeta^{13}}(X)=m_{\zeta^{26}}(X)=m_{\zeta^{21}}(X)=X^{5}+X^{4}+X^{3}+X+1 \\
& m_{\zeta^{15}}(X)=m_{\zeta^{30}}(X)=m_{\zeta^{29}}(X)=m_{\zeta^{27}}(X)=m_{\zeta^{23}}(X)=X^{5}+X^{3}+1 .
\end{aligned}
$$

Os polinônios geradores dos códigos $\mathrm{BCH}$ em sentido estrito, primitivo, com distrincia designada $\delta$ variando de 1 até 9 estão representados na tabela abaixo. 


\begin{tabular}{|c||l||l|}
\hline Valor de $\delta$ & Polinômio gerador & $\begin{array}{l}\text { Dimensão } \\
\text { do código }\end{array}$ \\
\hline 1 & $X^{1}-1$ & 30 \\
\hline 2 & $X^{5}+X^{2}+1$ & 26 \\
\hline 3 & $X^{5}+X^{2}+1$ & 26 \\
\hline 4 & $\left(X^{5}+X^{2}+1\right)\left(X^{5}+X^{4}+X^{3}+X^{2}+1\right)$ & 21 \\
\hline 5 & $\left(X^{5}+X^{2}+1\right)\left(X^{5}+X^{4}+X^{3}+X^{2}+1\right)$ & 21 \\
\hline 6 & $\left(X^{5}+X^{2}+1\right)\left(X^{5}+X^{4}+X^{3}+X^{2}+1\right)\left(X^{5}+\right.$ & 16 \\
& $\left.X^{4}+X^{2}+X+1\right)$ \\
\hline 7 & $\left(X^{5}+X^{2}+1\right)\left(X^{5}+X^{4}+X^{3}+X^{2}+1\right)\left(X^{5}+\right.$ & 16 \\
& $\left.X^{4}+X^{2}+X+1\right)$ \\
\hline 8 & $\left(X^{5}+X^{2}+1\right)\left(X^{5}+X^{4}+X^{3}+X^{2}+1\right)\left(X^{5}+\right.$ & 11 \\
& $\left.X^{4}+X^{2}+X+1\right)\left(X^{5}+X^{2}+X^{3}+X+1\right)$ & \\
\hline 9 & $\left(X^{5}+X^{2}+1\right)\left(X^{5}+X^{4}+X^{3}+X^{2}+1\right)\left(X^{5}+\right.$ & 11 \\
& $\left.X^{4}+X^{2}+X+1\right)\left(X^{5}+X^{2}+X^{3}+X+1\right)$ & \\
\hline
\end{tabular}

Teorema 4.1.7. O código BCH q-ário de comprimento $n$ e distância designada $\delta$ tem dimensão maior ou igual a $n-(\delta-1) o_{n}(q)$.

Demonstração: O corpo de decomposição do polinômio $X^{n}-1$ sobre $\mathbb{F}$ é $\mathbb{F}_{q^{m}}$, onde $m$ é a ordem multiplicativa de $q$ módulo $n\left(m=o_{n}(q)\right)$. Logo, $\operatorname{grau}\left(m_{\zeta^{b+i}}(X)\right) \leq m$, para $i=0, \ldots, \delta-2$. Assim, $\operatorname{grau}(g(X)) \leq m(\delta-1)$.

Portanto, a dimensão do código BCH é $n-\operatorname{grau}(g(X)) \geq n-m(\delta-1)$.

\subsection{Códigos Reed-Solomon}

Definição 4.2.1. Chama-se código Reed-Solomon o código $B C H$ de distância designada $\delta$ e de comprimento $n$ tal que $n=q-1$.

As raízes do polinômio $X^{n}-1$ são precisamente os elementos não nulos de $\mathbb{F}$ e temos

$$
X^{n}-1=\prod_{\alpha \in \mathbb{F}^{*}}(X-\alpha) .
$$

Portanto, se $\zeta$ é uma raiz $n$-ésima primitiva da unidade sobre $\mathbb{F}$ então

$$
m_{i}\left(X^{-}\right)=X-\zeta^{i}, \quad 0 \leq i \leq q-2 .
$$


Como resultado, um código Reed-Solomon com distância designada $\delta$ tem polinômio gerador

$$
g(X)=\left(X-\zeta^{b}\right)\left(X-\zeta^{b+1}\right) \cdots\left(X-\zeta^{b+\delta-2}\right)
$$

onde $b>0$.

Note que o polinômio gerador de um código Reed-Solomon de distância designada $\delta$ tem grau $(g(X))=\delta-1$.

Portanto,

$$
k=n-\operatorname{grau}(g(X))=n-\delta+1
$$

é a dimensão deste código.

Teorema 4.2.2. Códigos Reed-Solomon são códigos separáveis pela distância máxima (MDS). Além disso, a distância mínima do código Reed-Solomon é igual a sua distância designada.

Demonstração: Como os códigos Reed-Solomon são um caso particular dos códigos de $\mathrm{BCH}$, segue da Cota de $\mathrm{BCH}$ que a distância mínima de um código Reed-Solomon com distância designada $\delta$ satisfaz

$$
d \geq \delta=n-k+1 \text {. }
$$

A Cota de Singleton nos diz que

$$
d \leq n-k+1
$$

e portanto, $d=\delta=n-k+1$.

Portanto, códigos Reed-Solomon tem maior distância mínima possível entre os códigos $q$-ários de comprimento $n=q-1$ e dimensão $k=n-\delta+1$.

Exemplo 4.2.3. Sejam $q=8$ e $n=7$. Seja $\mathbb{F}$ o corpo finito com 8 elementos.

Uma raiz $\zeta$ do polinômio primitivo $X^{3}+X+1$ sobre $\mathbb{F}$ nos da uma raiz 7 -ésima primitiva da unidade sobre $\mathbb{F}$.

Para obtermos um códligo Reed-Solomon de dimensão 5, queremos que $k=n-\operatorname{grau}(g(X)) \Leftrightarrow 5=n-\operatorname{grau}(g(X)) \quad \Leftrightarrow \quad \operatorname{grau}(g(X))=7-5=2$

Portanto, a distância designada é $\delta=3$. Tomando $b=1$, temos que o polinômio gerador do código Reed-Solomon é

$$
\begin{aligned}
g(X) & =(X-\zeta)\left(X-\zeta^{2}\right) \\
& =X^{2}-\left(\zeta^{2}+\zeta\right) X+\zeta^{3} \\
& =X^{2}-\zeta^{4} X+\zeta^{3} .
\end{aligned}
$$




\section{Capítulo 5}

\section{Um caso particular}

Neste capítulo vamos estudar códigos cíclicos no caso em que seu comprimento e o número de elementos no corpo estão relacionados pela fórmula $q=\lambda n+1$.

\subsection{Idempotentes Primitivos}

Sejam $G=\langle a\rangle$ um grupo cíclico finito de ordem $n$ e $\mathbb{F}$ um corpo finito com $q$ elementos tal que $m d c(q, n)=1$.

Relembrando, se $\mathcal{I}$ é um código cíclico de comprimento $n$ com polinômio gerador $g(X)$ então $g(X)$ divide $X^{n}-1$ e, o polinômio $h(X)=\left(X^{n}-1\right) / g(X)$ é o polinômio de teste de $\mathcal{I}$, isto é, $\varphi \in \mathcal{I}$ se, e somente se, $h(X) \varphi(X)=$ 0 . Desde que $m d c(n, q)=1, X^{n}-1$ não tem raízes multiplas, portanto, $\operatorname{mdc}(g(X), h(X))=1$.

Lembremos que se $e(X)$ é o gerador idempotente do ideal $\mathcal{I}$ em $\mathcal{R}_{n}$ com polinômio gerador $g(X)$ então

$$
g(X)=\operatorname{mdc}\left(e(X), X^{n}-1\right)
$$

e, se o $\operatorname{grau}(g(X))=s$ então a dimensão do ideal $\mathcal{I}$ é igual a $n-s$ (Teoremas 1.3.7 e 3.3.4).

Assumiremos, a partir de agora, que o número $q$ de elementos de $\mathbb{F}$ e a dimensão $n$ de $\mathcal{R}_{n}$ estão relacionados pela fórmula $q=\lambda n+1$, para algum inteiro $\lambda>0$. 
Com esta condição afirmamos que $\mathbb{F}$ é um corpo de decomposição para o polinômio $X^{n}-1$. De fato, seja $E$ o corpo de decomposição para o polinômio $X^{n}-1$ sobre $\mathbb{F}_{p}$ com $|E|=p^{t}$. Logo $t$ é o menor inteiro positivo tal que $p^{t} \equiv 1(\bmod n)$. Como $q=\lambda n+1$ temos que $q \equiv 1(\bmod n)$, isto é, $p^{m} \equiv 1(\bmod n)$. Assim, temos que $t \leq m$, o que implica que $E \subset \mathbb{F}_{q}$, a menos de isomorfismo, ou seja, $\mathbb{F}_{q}$ contém todas as raízes do polinômio $X^{n}-1$.

Consideremos a representação $T_{i}: G \longrightarrow G L(1, \mathbb{F})$ definida por

$$
a^{j} \longmapsto \zeta^{i j}, \quad 1 \leq i \leq n
$$

onde $\zeta$ é uma raiz $n$-ésima primitiva da unidade em $\mathbb{F}$.

Observemos que as diferentes representações de $G$ sobre $\mathbb{F}$ de grau 1 são irredutíveis e não equivalentes, duas a duas. De fato, sejam $T_{i}$ e $T_{j}$ representações matriciais de $G$ sobre $\mathbb{F}$ de grau $1, i, j=1,2, \ldots, n$. Se $T_{i}$ e $T_{j}$ são equivalentes então existe um escalar $\gamma \in \mathbb{F}$, não nulo, tal que $T_{i}(x)=\gamma T_{j}(x) \gamma^{-1}$, isto é, $T_{i}(x)=T_{j}(x), \forall x \in G$. Como $G$ tem no máximo $|G|=n$ representações irredutíveis não equivalentes, segue que estas são todas as possíveis representações irredutíveis de $G$ sobre $\mathbb{F}$ e são todas de grau 1.

Logo, os caracteres irredutíveis de $G$ sobre $\mathbb{F}$ são as funções $\chi_{i}: G \longrightarrow \mathbb{F}$ definidas por

$$
a^{j} \longmapsto \zeta^{i j}, \quad 1 \leq i \leq n .
$$

Do Teorema 1.6.3, do capítulo 1, sabemos que os idempotentes centrais primitivos de $\mathbb{F} G$ são da seguinte forma:

$$
\varepsilon_{i}=\frac{1}{|G|} \sum_{x \in G} \chi_{i}(1) \chi_{i}\left(x^{-1}\right) x .
$$

Como $\chi_{i}\left(y^{-j}\right)=\zeta^{-i j}$, para todo $y \in G$ e $\chi_{i}(1)=1$, podemos escrever que

$$
\varepsilon_{i}=\frac{1}{|G|} \sum_{j=0}^{n-1}\left(\zeta^{i}\right)^{-j} a^{j} . \quad 0 \leq i \leq n-1 .
$$

Como conseqüência, obtemos imediatamente o seguinte resultado de Pruthi e Arora. 
Teorema 5.1.1. ([19], Teorema 2.1) Se $q=n \lambda+1$ então $\mathbb{F} G$ tem $n$ idempotentes primitivos dados por

$$
\varepsilon_{i}=\frac{1}{n} \sum_{j=0}^{n-1}\left(\zeta^{i}\right)^{-j} a^{j}, \quad 0 \leq i \leq n-1 .
$$

onde $\zeta$ é uma raiz n-ésima primitiva da unidade em $\mathbb{F}$.

Exemplo 5.1.2. (i) Sejam $G$ um grupo cíclico de ordem 2 e $\mathbb{F}$ um corpo finito com $q=2 \lambda+1$ elementos. Então, o inverso de 2 em $\mathbb{F}$ é $\lambda+1$ e os idempotentes primitivos em $\mathbb{F} G$ são:

$$
\begin{aligned}
& \varepsilon_{0}=(\lambda+1)(1+a) \\
& \varepsilon_{1}=(\lambda+1)(1-a) .
\end{aligned}
$$

(ii) Sejam $G$ um grupo cíclico de ordem 3 e $\mathbb{F}$ um corpo finito com $q=3 \lambda+1$ elementos. Então, o inverso de 3 em $\mathbb{F}$ é $2 \lambda+1$ e os idempotentes primitivos em $\mathbb{F} G$ são:

$$
\begin{aligned}
& \varepsilon_{0}=(2 \lambda+1)\left(1+a+a^{2}\right) \\
& \varepsilon_{1}=(2 \lambda+1)\left(1+\zeta^{2} a+\zeta a^{2}\right) \\
& \varepsilon_{2}=(2 \lambda+1)\left(1+\zeta a+\zeta^{2} a^{2}\right),
\end{aligned}
$$

onde $\zeta$ é uma raiz cúbica primitiva da unidade sobre $\mathbb{F}$.

(iii) Sejam $G$ um grupo cíclico de ordem 4 e $\mathbb{F}$ um corpo finito com $q=4 \lambda+1$ elementos. Então, o inverso de 4 em $\mathbb{F}$ é $q-\lambda$ e os idempotentes primitivos em $\mathbb{F} G$ são:

$$
\begin{aligned}
\varepsilon_{0} & =(q-\lambda)\left(1+a+a^{2}+a^{3}\right) \\
\varepsilon_{1} & =(q-\lambda)\left(1-\zeta a-a^{2}+\zeta a^{3}\right) \\
\varepsilon_{2} & =(q-\lambda)\left(1-a+a^{2}-a^{3}\right) \\
\varepsilon_{3} & =(q-\lambda)\left(1+\zeta a-a^{2}-\zeta a^{3}\right)
\end{aligned}
$$

onde $\zeta^{2}=-1$ em $\mathbb{F}$.

(iv) Sejam $G$ um grupo cíclico de ordem 6 e $\mathbb{Z}_{13}$ o corpo finito com 13 elementos. Então, o inverso de $6 \mathrm{em} \mathbb{Z}_{13}$ é 11 . Como $13=2 \times 6+1$ estamos nas condições do Teorema 5.1.1, $\log$ o os idempotentes primitivos em $\mathbb{Z}_{13} G$ são:

$$
\begin{aligned}
& \varepsilon_{0}=11\left(1+a+a^{2}+a^{3}+a^{4}+a^{5}\right) \\
& \varepsilon_{1}=11\left(1+10 a+9 a^{2}+12 a^{3}+3 a^{4}+4 a^{5}\right) \\
& \varepsilon_{2}=11\left(1+9 a+3 a^{2}+a^{3}+9 a^{4}+3 a^{5}\right) \\
& \varepsilon_{3}=11\left(1+12 a+a^{2}+12 a^{3}+a^{4}+12 a^{5}\right) \\
& \varepsilon_{4}=11\left(1+3 a+9 a^{2}+a^{3}+3 a^{4}+9 a^{5}\right) \\
& \varepsilon_{5}=11\left(1+4 a+3 a^{2}+12 a^{3}+9 a^{4}+10 a^{5}\right) .
\end{aligned}
$$


onde 2 é um gerador do grupo cíclico $\mathbb{Z}_{13}^{*}$ e 4 é uma raiz 6-ésima primitiva da unidade sobre $\mathbb{Z}_{13}$.

\subsection{Determinação dos códigos BCH e Reed- Solomon}

Nosso objetivo agora é determinarmos todos os possíveis códigos $\mathrm{BCH}$ e Reed-Solomon sobre o corpo $\mathbb{F}$ com $q=\lambda n+1$ elementos.

Seja $\varepsilon_{i}=\frac{1}{n} \sum_{j=0}^{n-1}\left(\zeta^{i}\right)^{-j} a^{j}$ um idempotente primitivo em $\mathbb{F} G$ e $\varepsilon_{i}(X)$ seu polinômio correspondente em $\mathcal{R}_{n}$. Mostraremos que o polinômio de teste do código cíclico gerado por $\varepsilon_{i}(X)$ é:

$$
h_{i}(X)=X-\zeta^{i}, \quad 0 \leq i \leq n-1
$$

ou equivalentemente, que o ideal gerado por $\varepsilon_{i}$ é o ideal anulado pelo elemento $h_{i}(a)=a-\zeta^{i}$, que denotaremos por $\mathcal{J}$.

De fato, vamos verificar se $h_{i}(a) \varepsilon_{i}(a)=0$, o que mostrará que $\varepsilon_{i}(a) \in \mathcal{J}$. Temos que

$$
\left(a-\zeta^{i}\right)\left(\sum_{j=0}^{n-1} \zeta^{-i j} a^{j}\right)=\sum_{j=0}^{n-1} \zeta^{-i j} a^{j+1}-\sum_{j=0}^{n-1} \zeta^{i(1-j)} a^{j}
$$

Tomado $j=h+1$ no segundo termo da subtração acima, obtemos

$$
\sum_{j=0}^{n-1} \zeta^{-i j} a^{j+1}-\sum_{h=0}^{n-1} \zeta^{-i h} a^{h+1}=0 .
$$

Consequentemente, $\mathbb{F} G \varepsilon_{i} \subset \mathcal{J}$. Do Teorema 1.2.5 sabemos que $\mathcal{J}$ é minimal, $\log \mathrm{F} G \varepsilon_{i}=\mathcal{J}$.

Portanto, o polinômio de teste do código cíclico gerado por $\varepsilon_{i}(X)$ é de fato $h_{i}(X)=X-\zeta^{i}$. Consequentemente, obtemos que o polinômio gerador desse código é dado por $g_{i}(X)=\left(X^{n}-1\right) / h_{i}(X)$.

Lema 5.2.1. Seja $\mathbb{F}$ um corpo de decomposição para o polinômio $X^{n}-1$. Então um código BCH tem distância designada $\delta$ se, e somente se, sua dimensio é igual a $n-\delta+1$. 
Demonstração: Sejam $2 \leq \delta \leq n$ e $b$ inteiros positivos. Consideremos $\mathcal{C}$ o código $\mathrm{BCH}$ com distância designada $\delta$. Segue, da definição, que $\mathcal{C}$ é gerado pelo mínimo multiplo comum dos polinômios minimais de $\zeta^{b}, \zeta^{b+1}, \ldots, \zeta^{b+\delta-2}$, onde $\zeta$ é uma raiz $n$-ésima primitiva da unidade sobre $\mathbb{F}$, e denotaremos esse polinômio por $g(X)$. Como $\mathbb{F}$ é um corpo de decomposição para o polinômio $X^{n}-1$ tem-se que $g(X)=\left(X-\zeta^{b}\right)\left(X-\zeta^{b+1}\right) \cdots$ $\left(X-\zeta^{b+\delta-2}\right)$. Portanto, o $\operatorname{grau}(g(X))=\delta-1$, e consequentemente, a dimensão de $\mathcal{C}$ é igual a $n-\delta+1$.

Reciprocamente, se a dimensão de $\mathcal{C}$ é igual a $n-\delta+1$ então o grau do polinômio gerador $g(X)$ é igual a $\delta-1$. Como $\mathbb{F}$ é um corpo de decomposição para o polinômio $X^{n}-1$ segue imediatamente que a distância designada desse código é $\delta$.

Com as notações acima, segue do Corolário 1.3.12, que o ideal gerado por

$$
f_{b} f_{b+1} \cdots f_{b+\delta-2}=\frac{X^{n}-1}{f_{b+\delta-1} f_{b+\delta} \cdots f_{b+n-1}}
$$

é

$$
\bigoplus_{i=b+\delta-1}^{b+n-1} \mathcal{M}_{i}
$$

e o idempotente gerador deste ideal é

$$
\varepsilon_{b+\delta-1}+\cdots+\varepsilon_{b+n-1}=1-\left(\varepsilon_{b}+\cdots+\varepsilon_{b+\delta-2}\right) .
$$

Assim, o idempotente gerador de um código $\mathrm{BCH}$, seu polinômio de teste e seu polinômio gerador são respectivamente:

$$
\begin{gathered}
\varepsilon_{b}^{\prime}=\sum_{j=b+\delta-1}^{b+n-1} \varepsilon_{j}, \\
h_{b}(X)=\prod_{j=b+\delta-1}^{b+n-1}\left(X-\zeta^{j}\right) \text { e } g_{b}(X)=\frac{X^{n}-1}{h_{b}(X)} .
\end{gathered}
$$

Vamos explicitar toclos os códigos BCH de uma dada dimensão $k$. Neste caso, sua distância designada é $\delta=n-k+1$. O idempotente, o polinômio de teste e o polinômio gerador de um código $\mathrm{BCH}$ de parâmetros $(n, k)$ sobre F são dados pelas fórmulas:

$$
\hat{\varepsilon}_{b}^{\prime}=\sum_{j=b+n-k}^{b+n-1} \varepsilon_{j},
$$




$$
h_{b}(X)=\prod_{j=b+n-k}^{b+n-1}\left(X-\zeta^{j}\right) \quad \text { e } \quad g_{b}(X)=\frac{X^{n}-1}{h_{b}(X)}, \quad 1 \leq b \leq n
$$

onde deve-se lembrar que os índices da somatória e do produto são tomados em módulo $n$.

Assim, para encontrarmos os idempotentes geradores dos códigos $\mathrm{BCH}$ de dimensão $k$ devemos somar $k$ consecutivos idempotentes primitivos.

Exemplo 5.2.2. Sejam $G=\langle a\rangle, a^{6}=1$, um grupo cíclico de ordem 6 e $\mathbb{Z}_{7}$ o corpo finito com 7 elementos.

Lembremos que, se $\lambda=1$ então $q=n+1$, ou seja, o código $\mathrm{BCH}$ sobre $\mathbb{F}_{q}$ recebe o nome de Reed-Solomon. Sabemos, do Capítulo 4, que sua distância designada é igual a sua distância mínima.

Vamos construir os possíveis códigos Reed-Solomon de comprimento 6 e dimensão 2 sobre $\mathbb{Z}_{7}$.

Temos que o inverso de 6 em $\mathbb{Z}_{7}$ é o próprio 6 .

Note que uma raiz 6-ésima primitiva da unidade sobre $\mathbb{Z}_{7}$ é um elemento primitivo de $\mathbb{Z}_{7}$, ou seja, é um gerador do grupo cíclico $\mathbb{Z}_{7}^{*}$. É fácil ver que 3 é uma raiz 6-ésima primitiva da unidade sobre $\mathbb{Z}_{7}$ e portanto $\mathbb{Z}_{7}^{*}=\langle\overline{3}\rangle$.

Observemos que estamos nas condições do Teorema 5.1.1, porque $7=1 \times 6+1$. Como a dimensão desse código é igual a 2 segue que o grau dos possíveis polinômios geradores é igual a 4. Do Lema 5.2.1 tem-se que $\delta=5$, ou seja, $d=5$.

Os elementos idempotentes de $\mathbb{Z}_{7} G$ com seus polinômios de teste correspondentes $h(X)$ são:

\begin{tabular}{|l||l|}
\hline Idlempotentes Primitivos & $\mathrm{h}(\mathrm{X})$ \\
\hline$\varepsilon_{0}=6\left(1+a+a^{2}+a^{3}+a^{4}+a^{5}\right)$ & $X-1$ \\
\hline$\varepsilon_{1}=6\left(1+5 a+4 a^{2}+6 a^{3}+2 a^{4}+3 a^{5}\right)$ & $X-3$ \\
\hline$\varepsilon_{2}=6\left(1+4 a+2 a^{2}+a^{3}+4 a^{4}+2 a^{5}\right)$ & $X-2$ \\
\hline$\varepsilon_{3}=6\left(1+6 a+a^{2}+6 a^{3}+a^{4}+6 a^{5}\right)$ & $X-6$ \\
\hline$\varepsilon_{4}=6\left(1+2 a+4 a^{2}+a^{3}+2 a^{4}+4 a^{5}\right)$ & $X-4$ \\
\hline$\varepsilon_{5}=6\left(1+3 a+2 a^{2}+6 a^{3}+4 a^{4}+5 a^{5}\right)$ & $X-5$ \\
\hline
\end{tabular}

Os idempotentes geradores $e(X)$, os polinômios de teste $h(X)$ e os polinômios geradores $g(X)$ correspondentes aos códigos Reed-Solomon cujos parâmetros são $(6,2)$ são: 


\begin{tabular}{|l||l||l|}
\hline Idempotentes & Polinômio de Teste & Polinômio Gerador \\
\hline$e_{1}=\varepsilon_{5}+\varepsilon_{0}$ & $X^{2}+X+5$ & $X^{4}+6 X^{3}+3 X^{2}+2 X+4$ \\
\hline$e_{2}=\varepsilon_{0}+\varepsilon_{1}$ & $X^{2}+3 X+3$ & $X^{4}+4 X^{3}+6 X^{2}+5 X+2 \quad(*)$ \\
\hline$e_{3}=\varepsilon_{1}+\varepsilon_{2}$ & $X^{2}+2 X+6$ & $X^{4}+5 X^{3}+5 X^{2}+2 X+1$ \\
\hline$e_{4}=\varepsilon_{2}+\varepsilon_{3}$ & $X^{2}+6 X+5$ & $X^{4}+X^{3}+3 X^{2}+5 X+4$ \\
\hline$e_{5}=\varepsilon_{3}+\varepsilon_{4}$ & $X^{2}+4 X+3$ & $X^{1}+3 X^{3}+6 X^{2}+2 X+2$ \\
\hline$e_{0}=\varepsilon_{4}+\varepsilon_{5}$ & $X^{2}+5 X+6$ & $X^{4}+2 X^{3}+5 X^{2}+5 X+1$ \\
\hline
\end{tabular}

Exemplo 5.2.3. Sejam $G=\langle a\rangle, a^{5}=1$, um grupo cíclico de ordem 5 e $\mathbb{F}_{81}$ o corpo finito com 81 elementos.

Vamos construir os possíveis códigos $\mathrm{BCH}$ de comprimento 5 e dimensão 2 sobre $\mathbb{F}_{81}$.

Seja $\xi$ uma raiz 5 -ésima primitiva da unidade sobre $\mathbb{F}_{81}$.

Logo, estamos nas condições do Teorema 5.1.1, porque $81=16 \times 5+1$. Como a dimensão desse código é igual a 2 segue que o grau dos polinômios geradores é igual a 3. Do Lema 5.2.1 tem-se que $\delta=4$.

Assim, os elementos idempotentes de $\mathbb{F}_{81} G$ com seus polinômios de teste correspondentes $h(X)$ são:

\begin{tabular}{|l||l|}
\hline Idempotentes Primitivos & $\mathrm{h}(\mathrm{X})$ \\
\hline$\varepsilon_{0}=\frac{1}{5}\left(1+a+a^{2}+a^{3}+a^{4}\right)$ & $X-1$ \\
\hline$\varepsilon_{1}=\frac{1}{5}\left(1+\xi^{4} a+\xi^{3} a^{2}+\xi^{2} a^{3}+\xi a^{4}\right)$ & $X-\xi$ \\
\hline$\varepsilon_{2}=\frac{1}{5}\left(1+\xi^{3} a+\xi a^{2}+\xi^{4} a^{3}+\xi^{2} a^{4}\right)$ & $X-\xi^{2}$ \\
\hline$\varepsilon_{3}=\frac{1}{5}\left(1+\xi^{2} a+\xi^{4} a^{2}+\xi a^{3}+\xi^{3} a^{4}\right)$ & $X-\xi^{3}$ \\
\hline$\varepsilon_{4}=\frac{1}{5}\left(1+\xi a+\xi^{2} a^{2}+\xi^{3} a^{3}+\xi^{4} a^{4}\right)$ & $X-\xi^{4}$ \\
\hline
\end{tabular}

Seja $\zeta$ um elemento primitivo de $\mathbb{F}_{81}$ satisfazendo o polinômio primitivo $X^{4}+$ $X+2$. Como $\xi$ é uma raiz 5-ésima primitiva da unidade temos que $\xi=\zeta^{i}$. As$\operatorname{sim}, \xi^{5}=\left(\zeta^{i}\right)^{5}=1$ então $5 i \mid 80$ ou seja, $i \mid 16$. É fácil ver que $i=16$, isto é, $\xi=\zeta^{16}$.

Os idempotentes geradores $e(X)$, os polinômios de teste $h(X)$ e os polinômios geradores $g(X)$ correspondentes ao códligo BCH cujos parâmetros são $(5,2)$ são:

\begin{tabular}{|l||l||ll|}
\hline Idempotentes & Polinômio de teste & Polinômio Gerador \\
\hline$e_{1}=\varepsilon_{4}+\varepsilon_{0}$ & $X^{2}+\zeta^{2} X+\zeta^{64}$ & $X^{3}+\zeta^{42} X^{2}+\zeta^{34} X+\zeta^{56}$ \\
\hline$e_{2}=\varepsilon_{0}+\varepsilon_{1}$ & $X^{2}+\zeta^{18} X+\zeta^{16}$ & $X^{3}+\zeta^{58} X^{2}+\zeta^{66} X+\zeta^{24} \quad(*)$ \\
\hline$e_{3}=\varepsilon_{1}+\varepsilon_{2}$ & $X^{2}+\zeta^{34} X+\zeta^{18}$ & $X^{3}+\zeta^{74} X^{2}+\zeta^{18} X+\zeta^{72}$ \\
\hline$e_{1}=\varepsilon_{2}+\varepsilon_{3}$ & $X^{2}+\zeta^{50} X+1$ & $X^{3}+\zeta^{10} X^{2}+\zeta^{50} X+\zeta^{40}$ \\
\hline$e_{0}=\varepsilon_{3}+\varepsilon_{1}$ & $X^{2}+\zeta^{66} X+\zeta^{-32}$ & $X^{3}+\zeta^{26} X^{2}+\zeta^{-2} X+\zeta^{8}$ \\
\hline
\end{tabular}


Nós desenvolvemos aqui os mesmos exemplos que aparecem no artigo de Pruthi e Arora [19]. Os idempotentes primitivos encontrados são os mesmos que aparecem naquele artigo. Notamos, porém, que no caso do Exemplo 5.2.2 eles deixaram de encontrar o código de dimensão 2 gerado por $X^{4}+4 X^{3}+$ $6 X^{2}+5 X+2$, e no caso do Exemplo 5.2.3, o código de dimensão 2 gerado por $X^{3}+\zeta^{58} X^{2}+\zeta^{66} X+\zeta^{24}$. Esta omissão clecorre do fato de que esses autores deixaram de tomar índices das somátorias e do produto em módulo $n$ não fazendo o índice $i$ percorrer todos os inteiros no intervalo de 0 a $n-1$. 


\subsection{Apêndice}

Notamos que as bibliografias básicas da Teoria de códigos apresentam a tabela dos elementos do corpo $\mathbb{F}_{81}$ usando o polinômio primitivo $X^{4}+$ $X^{3}+2$. Nós refizemos esta tabela, usando o polinômio primitivo $X^{4}+$ $X+2$, para que os nossos elementos idempotentes primitivos, idempotentes, polinômios geradores e polinômios de teste pudessem ser comparados com os encontrados por Pruthi e Arora em [19, Exemplo4.3], que usaram esse polinômio. Transcreveremos essa tabela a seguir.

\begin{tabular}{|c|c|c|c|}
\hline \multicolumn{4}{|c|}{ Tabela dos elementos do corpo finito $\mathbb{F}_{81}$} \\
\hline Expoente & Elemento em $\mathbb{F}_{81}$ & Expoente & Elemento em $\mathbb{F}_{81}$ \\
\hline 0 & 1 & 40 & 2 \\
\hline 1 & $\zeta$ & 41 & $2 \zeta$ \\
\hline 2 & $\zeta^{2}$ & 42 & $2 \zeta^{2}$ \\
\hline 3 & $\zeta^{3}$ & 43 & $2 \zeta^{3}$ \\
\hline 4 & $2 \zeta+1$ & 44 & $\zeta+2$ \\
\hline 5 & $2 \zeta^{2}+\zeta$ & 45 & $\zeta^{2}+2 \zeta$ \\
\hline 6 & $2 \zeta^{3}+\zeta^{2}$ & 46 & $\zeta^{3}+2 \zeta^{2}$ \\
\hline 7 & $\zeta^{3}+\zeta+2$ & 47 & $2 \zeta^{3}+2 \zeta+1$ \\
\hline 8 & $\zeta^{2}+\zeta+1$ & 48 & $2 \zeta^{2}+2 \zeta+2$ \\
\hline 9 & $\zeta^{3}+\zeta^{2}+\zeta$ & 49 & $2 \zeta^{3}+2 \zeta^{2}+2 \zeta$ \\
\hline 10 & $\zeta^{3}+\zeta^{2}+2 \zeta+1$ & 50 & $2 \zeta^{3}+2 \zeta^{2}+\zeta+2$ \\
\hline 11 & $\zeta^{3}+2 \zeta^{2}+1$ & 51 & $2 \zeta^{3}+\zeta^{2}+2$ \\
\hline 12 & $2 \zeta^{3}+1$ & 52 & $\zeta^{3}+2$ \\
\hline 13 & $2 \zeta+2$ & 53 & $\zeta+1$ \\
\hline 14 & $2 \zeta^{2}+2 \zeta$ & 54 & $\zeta^{2}+\zeta$ \\
\hline 15 & $2 \zeta^{3}+2 \zeta^{2}$ & 55 & $\zeta^{3}+\zeta^{2}$ \\
\hline 16 & $2 \zeta^{3}+\zeta+2$ & 56 & $\zeta^{3}+2 \zeta+1$ \\
\hline 17 & $\zeta^{2}+2$ & 57 & $2 \zeta^{2}+1$ \\
\hline 18 & $\zeta^{3}+2 \zeta$ & 58 & $2 \zeta^{3}+\zeta$ \\
\hline 19 & $2 \zeta^{2}+2 \zeta+1$ & 59 & $\zeta^{2}+\zeta+2$ \\
\hline 20 & $2 \zeta^{3}+2 \zeta^{2}+\zeta$ & 60 & $\zeta^{3}+\zeta^{2}+2 \zeta$ \\
\hline 21 & $2 \zeta^{3}+\zeta^{2}+\zeta+2$ & 61 & $\zeta^{3}+2 \zeta^{2}+2 \zeta+1$ \\
\hline 22 & $\zeta^{3}+\zeta^{2}+2$ & 62 & $2 \zeta^{3}+2 \zeta^{2}+1$ \\
\hline 23 & $\zeta^{3}+\zeta+1$ & 63 & $2 \zeta^{3}+2 \zeta+2$ \\
\hline 24 & $\zeta^{2}+1$ & 64 & $2 \zeta^{2}+2$ \\
\hline 25 & $\zeta^{3}+\zeta$ & 65 & $2 \zeta^{3}+2 \zeta$ \\
\hline 26 & $\zeta^{2}+2 \zeta+1$ & 66 & $2 \zeta^{2}+\zeta+2$ \\
\hline 27 & $\zeta^{3}+2 \zeta^{2}+\zeta$ & 67 & $2 \zeta^{3}+\zeta^{2}+2 \zeta$ \\
\hline 28 & $2 \zeta^{3}+\zeta^{2}+2 \zeta+1$ & 68 & $\zeta^{3}+2 \zeta^{2}+\zeta+2$ \\
\hline 29 & $\zeta^{3}+2 \zeta^{2}+2 \zeta+2$ & 69 & $2 \zeta^{3}+\zeta^{2}+\zeta+1$ \\
\hline 30 & $2 \zeta^{3}+2 \zeta^{2}+\zeta+1$ & 70 & $\zeta^{3}+\zeta^{2}+2 \zeta+2$ \\
\hline 31 & $2 \zeta^{3}+\zeta^{2}+2 \zeta+2$ & 71 & $\zeta^{3}+2 \zeta^{2}+\zeta+1$ \\
\hline 32 & $\zeta^{3}+2 \zeta^{2}+2$ & 72 & $2 \zeta^{3}+\zeta^{2}+1$ \\
\hline 33 & $2 \zeta^{3}+\zeta+1$ & 73 & $\zeta^{3}+2 \zeta+2$ \\
\hline 34 & $\zeta^{2}+2 \zeta+2$ & 74 & $2 \zeta^{2}+\zeta+1$ \\
\hline 35 & $\zeta^{3}+2 \zeta^{2}+2 \zeta$ & 75 & $2 \zeta^{3}+\zeta^{2}+\zeta$ \\
\hline 36 & $2 \zeta^{3}+2 \zeta^{2}+2 \zeta+1$ & 76 & $\zeta^{3}+\zeta^{2}+\zeta+2$ \\
\hline 37 & $2 \zeta^{3}+2 \zeta^{2}+2 \zeta+2$ & 77 & $\zeta^{3}+\zeta^{2}+\zeta+1$ \\
\hline 38 & $2 \zeta^{3}+2 \zeta^{2}+2$ & 78 & $\zeta^{3}+\zeta^{2}+1$ \\
\hline 39 & $2 \zeta^{3}+2$ & 79 & $\zeta^{3}+1$ \\
\hline
\end{tabular}




\section{Capítulo 6}

\section{Códigos Cíclicos Minimais}

Neste capítulo assumiremos que $G$ é um grupo cíclico finito, $\mathbb{F}$ um corpo finito com $|\mathbb{F}|=q$ elementos e $q$ é uma potência de um primo tal que $\operatorname{mdc}(q,|G|)=1$.

\subsection{Preliminares}

Seja $H$ um subgrupo do grupo $G$. Considere

$$
\widetilde{H}=\frac{1}{|H|} \sum_{h \in H} h .
$$

Como $|H|$ divide $|G|$ e $m d c(q,|G|)=1$, segue que $\widetilde{H}$ está bem definida e do Lema 1.4.12, temos que $\widetilde{H}$ é um idempotente de $\mathbb{F} G$.

Lema 6.1.1. Seja $A=\langle a\rangle$ um grupo cíclico de ordem $p^{n}, n \geq 1$. Considere

$$
A=A_{0} \supset A_{1} \supset \cdots \supset A_{n}=\{1\}
$$

a cadeia descendente de todos os subgrupo cíclicos de $A$, onde $A_{i}=\left\langle a^{p^{i}}\right\rangle$ e $\left|A_{i}\right|=p^{n-i}$. Se $1 \leq i \leq j$ então $\widetilde{A_{i}} \widetilde{A_{j}}=\widetilde{A_{i}}$.

Demonstração: Consideremos o produto

$$
\widetilde{A_{i}} \widetilde{A_{j}}=\left(\frac{1}{\left|A_{i}\right|} \widehat{A_{i}}\right)\left(\frac{1}{\left|A_{j}\right|} \widehat{A_{j}}\right)=\frac{1}{\left|A_{i}\right|\left|A_{j}\right|} \widehat{A_{i}} \widehat{A_{j}}
$$


Como $A_{i} \supset A_{j}$ segue que

$$
\widetilde{A_{i}} \widetilde{A_{j}}=\frac{1}{\left|A_{i}\right|\left|A_{j}\right|}\left|A_{j}\right| \widehat{A_{i}}=\frac{1}{\left|A_{i}\right|} \widehat{A_{i}}=\widetilde{A_{i}}
$$

Lema 6.1.2. Seja $p$ um primo racional e seja $A=\langle a\rangle$ um grupo cíclico de ordem $p^{n}, n \geq 1$. Considere

$$
A=A_{0} \supset A_{1} \supset \cdots \supset A_{n}=\{1\}
$$

a cadeia descendente de todos os subgrupo cíclicos de $A$, onde $A_{i}=\left\langle a^{p^{i}}\right\rangle$. Então os elementos

$$
e_{0}=\widetilde{A} \quad e \quad e_{i}=\widetilde{A_{i}}-\widetilde{A_{i-1}}, \quad 1 \leq i \leq n
$$

formam um conjunto de idempotentes primitivos na álgebra de grupo dos racionais $\mathbb{Q} A$ tal que

$$
e_{0}+e_{1}+\cdots+e_{n}=1
$$

Demonstração: Segue diretamente do Corolário do Teorema de PerlisWalker 1.4 .8 que

$$
\mathbb{Q} A \cong \bigoplus_{i=0}^{n} \mathbb{Q}\left(\zeta_{p^{i}}\right)
$$

onde $\zeta_{p^{i}}$ denota uma raiz primitiva da unidade de ordem $p^{i}$ e temos precisamente $n+1$ idempotentes primitivos na álgebra de grupo $\mathbb{Q} A$.

Vamos mostrar que $e_{0}$ e $e_{i}, 1 \leq i \leq n$, são os idempotentes primitivos de $\mathbb{Q} A$.

Note que, do Lema 6.1.1 segue que $e_{0} \widetilde{A_{i}}=e_{0}$, para $i \geq 1$.

Sabemos que $e_{0}$ é um idempotente de $\mathbb{Q} A$ e temos que

$$
e_{0} e_{i}=e_{0}\left(\widetilde{A_{i}}-\widetilde{A_{i-1}}\right)=e_{0} \widetilde{A_{i}}-e_{0} \widetilde{A_{i-1}}=e_{0}-e_{0}=0 .
$$

Agora, mostraremos que os elementos $c_{i}, 1 \leq i \leq n$, são idempotentes de $\mathbb{Q} A$, ortogonais dois a clois. 
Seja $i \leq j$ e consideremos o produto:

$$
\begin{aligned}
e_{i} e_{j} & \left.=\widetilde{\left(A_{i}\right.}-\widetilde{A_{i-1}}\right)\left(\widetilde{A_{j}}-\widetilde{A_{j-1}}\right) \\
& =\widetilde{A_{i}} \widetilde{A_{j}}-\widetilde{A_{i}} \widetilde{A_{j-1}}-\widetilde{A_{i-1}} \widetilde{A_{j}}+\widetilde{A_{i-1}} \widetilde{A_{j-1}} \\
& =\widetilde{A_{i}}-\widetilde{A_{i}} \widetilde{A_{j-1}}-\widetilde{A_{i-1}}+\widetilde{A_{i-1}} \\
& =\widetilde{A_{i}}-\widetilde{A_{i} A_{j-1}} .
\end{aligned}
$$

Se $i<j$ então $e_{i} e_{j}=\widetilde{A_{i}}-\widetilde{A_{i}} \widetilde{A_{j-1}}=\widetilde{A_{i}}-\widetilde{A_{i}}=0$.

Se $i=j$ então $e_{i} e_{j}=\widetilde{A_{i}}-\widetilde{A_{i}} \widetilde{A_{j-1}}=\widetilde{A_{i}}-\widetilde{A_{i-1}}=e_{i}$.

Agora,

$$
\begin{aligned}
e_{0}+e_{1}+\ldots+e_{n} & =\widetilde{A_{0}}+\widetilde{A_{1}}-\widetilde{A_{0}}+\widetilde{A_{2}}-\widetilde{A_{1}}+\cdots+\widetilde{A_{n}}-\widetilde{A_{n-1}} \\
& =\widetilde{A_{n}} \\
& =1 .
\end{aligned}
$$

Portanto, $\left\{e_{0}, e_{1}, \ldots, e_{n}\right\}$ é o conjunto dos $n+1$ idempotentes primitivos de $\mathbb{Q} A$. De fato, se pudermos escrever $e_{i}=e_{i}^{\prime}+e_{i}^{\prime \prime}$, para algum índice $i$, onde $e_{i}^{\prime}, e_{i}^{\prime \prime}$ são idempotentes ortogonais, não nulos, teriamos $n+2$ idempotentes ortogonais cuja soma desses é igual a 1, uma contradição, uma vez que mostramos que há exatamente $n+1$ idempotentes ortogonais cuja soma desses é igual a 1.

Observamos que este método produz idempotentes primitivos de $\mathbb{Q} A$ mas, não em geral, sobre corpos finitos.

Exemplo 6.1.3. Sejam $G$ o grupo cíclico de ordem 3 e $\mathbb{F}$ o corpo finito com 7 elementos então a fatoração do polinômio $X^{3}-1$ sobre $\mathbb{F}$ é igual a $(X-1)(X-2)(X-4)$. Então, a álgebra de grupo $\mathbb{F} G$ é

$$
\mathbb{F} G \cong \frac{\mathbb{F}[X]}{\left\langle X^{3}-1\right\rangle} \cong \frac{\mathbb{F}[X]}{\langle X-1\rangle} \oplus \frac{\mathbb{F}[X]}{\langle X-2\rangle} \oplus \frac{\mathbb{F}[X]}{\langle X-4\rangle} .
$$

Por outro lado,

$$
\mathbb{Q} G \cong \mathbb{Q} \oplus \mathbb{Q}\left(\zeta_{3}\right)
$$

onde $\zeta_{3}$ denota uma raiz cúbica primitiva cla uniclade.

Portanto, a álgebra de grupo racional de $G$ sobre $\mathbb{Q}$ contém apenas 2 idempotentes primitivos não triviais enquanto a álgebra de grupo, do mesmo grupo, sobre F contém 3 . 
Observemos que, do Teorema 2.2.1 segue que o número de componentes simples de $\mathbb{F} A$ é igual ao número de $q$-classes ciclotômicas de $A$. Assim, quando $\mathcal{S}_{h}=\mathcal{G}_{h}$ teremos que o número de componentes simples de $\mathbb{F} A$ será igual ao número de subgrupos cíclicos de $A$. Portanto, o número de componentes simples de $\mathbb{F} A$ será igual ao de $\mathbb{Q} A$. Logo, poderemos aplicar o Lema 6.1.2 para encontrarmos o conjunto de idempotentes primitivos de $\mathbb{F} A$.

Assim, queremos encontrar condições para que $\mathcal{S}_{h}=\mathcal{G}_{h}$.

Corolário 6.1.4. Sejam $\mathbb{F}$ um corpo finito com $q$ elementos, $A$ um grupo cíclico de ordem $p^{n}$, tal que $m d c\left(q, p^{n}\right)=1$. Então, o conjunto de idempotentes primitivos dados no Lema 6.1.2 é o conjunto de idempotentes primitivos de $\mathbb{F} A$ se e somente se vale uma das seguintes afirmações.

(i) $p=2, n=1$ e qué impar ou $n=2$ e $q \equiv 3 \quad(\bmod 4)$;

(ii) p é um primo ímpar e $o(\bar{q})=\phi\left(p^{n}\right)$ em $U\left(\mathbb{Z}_{p^{n}}\right)$;

Demonstração: Se $\mathbb{F} A$ contém $n+1$ idempotentes primitivos então segue o Teorema 2.2.1 que há $n+1 q$-classes ciclotômicas, donde, $\mathcal{S}_{h}=\mathcal{G}_{h}$. Assim, do Corolário 2.2.3 seguem imediatamente os itens (i) e (ii).

Reciprocamente, se valem os itens $(i)$ e (ii) então, do Corolário 2.2.3, vem que $\mathcal{S}_{h}=\mathcal{G}_{h}$. Portanto, da observação acima, segue o resultado.

Como conseqüência imediata, obtemos o seguinte resultado de Pruthi e Arora.

Teorema 6.1.5. ([19], Teorema 3.5) Sejam $\mathbb{F}$ um corpo finito com $q$ elementos e A um grupo cíclico de ordem $p^{n}$ tal que o $(\bar{q})=\phi\left(p^{n}\right)$ em $U\left(\mathbb{Z}_{p^{n}}\right)$. Seja

$$
A=A_{0} \supset A_{1} \supset \cdots \supset A_{n}=\{1\}
$$

a cadeia descendente de todos os subgrupos cíclicos de $A$, onde $A_{i}=\left\langle a^{p^{i}}\right\rangle$. Então, o conjunto dos idempotentes primitivos de $\mathbb{F} A$ é dado por

$$
e_{0}=\frac{1}{p^{n}}\left(\sum_{h \in A} h\right) \quad \text { e } e_{i}=\widetilde{A_{i}}-\widetilde{A_{i-1}}, \quad 1 \leq i \leq n .
$$

Agora, consideremos o grupo cíclico $G$ de ordem $2 p^{n}, \mathbb{F}$ o corpo finito com $q$ elementos, onde $q$ é uma potência de $u$ mrimo ímpar e $m d c(q, p)=1$. O cálculo dos idempotentes primitivos da álgebra de grupo $\mathbb{F} G$ segue facilmente dos resultados anteriores, como veremos a seguir. 
Teorema 6.1.6. ([1], Teorema 2.6) Sejam $\mathbb{F}$ um corpo finito com q elementos e $G$ um grupo cíclico de ordem $2 p^{n}$, $p$ um primo ímpar, tal que o $(\bar{q})=\phi\left(p^{n}\right)$ em $U\left(\mathbb{Z}_{2 p^{n}}\right)$. Escrevendo $G=C \times A$, onde $A$ é o p-subyrupo de Sylow de $G$ e $C=\{1, t\}$ é seu 2-subgrupo de Sylow. Se $e_{i}$ denota os idempotentes primitivos de $\mathbb{F} A, 1 \leq i \leq n$, então os idempotentes primitivos de $\mathbb{F} G$ são:

$$
\frac{(1+t)}{2} e_{i} \quad e \quad \frac{(1-t)}{2} e_{i}, \quad 1 \leq i \leq n .
$$

Demonstração: Como o $(\bar{q})=\phi\left(p^{n}\right)$ em $U\left(\mathbb{Z}_{2 p^{n}}\right)$ tem-se que $\overline{\bar{q}} \in \mathbb{Z}_{p^{n}}$ é um gerador do grupo cíclico $U\left(\mathbb{Z}_{p^{n}}\right)$. Então, segue do Teorema 6.1.5, que o conjunto dos idempotentes primitivos de $\mathbb{F} A$ é dado por

$$
e_{0}=\frac{1}{p^{n}}\left(\sum_{h \in A} h\right) \quad \text { e } \quad e_{i}=\widetilde{A_{i}}-\widetilde{A_{i-1}}, \quad 1 \leq i \leq n .
$$

Também sabemos que os idempotentes de $\mathbb{F} C$ são:

$$
\frac{(t+1)}{2} \text { e } \frac{(t-1)}{2}
$$

Utilizando a Proposição 1.4.13 temos que

$$
\mathbb{F} C=\mathbb{F} C \frac{(1+t)}{2} \oplus \mathbb{F} C \frac{(1-t)}{2} \cong \mathbb{F} \oplus \mathbb{F} .
$$

Assim, temos que $\mathbb{F} C(1+t) / 2 \cong \mathbb{F}$ e, neste isomorfismo o elemento $1 \in \mathbb{F}$ corresponde ao elemento $(1+t) / 2 \in \mathbb{F} C(1+t) / 2$.

Analogamente, $\mathbb{F} C(1-t) / 2 \cong \mathbb{F}$ e, neste isomorfismo o elemento $1 \in \mathbb{F}$ corresponde ao elemento $(1-t) / 2 \in \mathbb{F} C(1-t) / 2$.

Assim, utilizando a Proposição 1.4.3, segue que

$$
\begin{aligned}
\mathbb{F} G \cong(\mathbb{F} C) A & =\left(\mathbb{F} C \frac{(1+t)}{2} \oplus \mathbb{F} C \frac{(1-t)}{2}\right) A \\
& \cong\left(\mathbb{F} C \frac{(1+t)}{2}\right) A \oplus\left(\mathbb{F} C \frac{(1-t)}{2}\right) A \\
& \cong \mathbb{F} A \oplus \mathbb{F} A .
\end{aligned}
$$

Logo, $\mathbb{F} A \cong\left(\mathbb{F} C \frac{(1+t)}{2}\right) A$ e, neste isomorfismo o elemento $e_{i}=1 e_{i} \in \mathbb{F} A$ corresponde ao elemento $\frac{(1+t)}{2} e_{i} \in\left(\mathbb{F} C \frac{(1+t)}{2}\right) A$. E temos também que, $\mathbb{F} A \cong$ $\left(\mathbb{F} C \frac{(1-t)}{2}\right) A$ e, neste isomorfismo o elemento $e_{i}=1 e_{i} \in \mathbb{F} A$ corresponde ao elemento $\frac{(1-t)}{2} e_{i} \in\left(\mathbb{F} C \frac{(1-t)}{2}\right) A$. 
Portanto, os $2 n+2$ idempotentes primitivos de $\mathbb{F} G$ são

$$
\frac{(1+t)}{2} e_{i} \quad \text { e } \quad \frac{(1-t)}{2} e_{i}, \quad 1 \leq i \leq n
$$

Os resultados de Arora e Pruthi [1] e [19] são apresentados em termos de classes ciclotômicas e as expressões dos idempotentes parecem bem mais complexas que as obtidas acima.

No apêndice deste capítulo mostraremos que os resultados obtidos nesta seção na verdade coincidem com os destes autores.

\subsection{Códigos Minimais}

Nesta seção iremos explicitar as expressões dos geradores dos códigos cíclicos minimais de comprimento $p^{n}$ e $2 p^{n}$ a partir dos idempotentes primitivos encontrados nos Teoremas 6.1.5 e 6.1.6.

No Teorema 1.3.7 provamos que se $e_{i}(X) \in \mathbb{F}[X]$ é um polinômio tal que $e_{i}(a)=\varepsilon_{i}$, onde $\varepsilon_{i}$ é um idempotente de $\mathbb{F} G$, então o polinômio gerador do ideal $\mathcal{I}_{i}$ de $\mathbb{F}[X] /\left\langle X^{n}-1\right\rangle$ gerado pelo idempotente $e_{i}(X)$ é dado por:

$$
g_{i}(X)=m d c\left(e_{i}(X), X^{n}-1\right), \quad 0 \leq i \leq n .
$$

\section{Geradores do código cíclico de comprimento $p^{n}$}

Vamos encontrar as expressões dos polinômios $g_{i}(X)$, que são os polinômios geradores dos códigos cíclicos minimais de comprimento $p^{n}$ : gerados pelos iclempotentes encontrados no Teorema 6.1.5.

Note que: 


$$
\begin{aligned}
e_{i}(X) & =\frac{1}{p^{n-i}} \sum_{j=0}^{p^{n-i}-1} X^{j p^{i}}-\frac{1}{p^{n-i+1}} \sum_{j=0}^{p^{n-i+1}-1} X^{j p^{i-1}} \\
& =\frac{1}{p^{n-i+1}}\left[p \sum_{j=0}^{p^{n-i}-1} X^{j p^{i}}-\sum_{j=0}^{p^{n-i+1}-1} X^{j p^{i-1}}\right] \\
& =\frac{1}{p^{n-i+1}}\left[(p-1) \sum_{j=0}^{p^{n-i}-1} X^{j p^{i}}-\left(\sum_{j=1}^{p-1} X^{j p^{i-1}}\right)\left(\sum_{j=0}^{p^{n-i}-1} X^{j p^{i}}\right)\right] \\
& =\frac{1}{p^{n-i+1}}\left[\left(p-\sum_{j=0}^{p-1} X^{j p^{i-1}}\right)\left(\sum_{j=0}^{p^{n-i}-1} X^{j p^{i}}\right)\right]
\end{aligned}
$$

Como toda raiz do polinômio $X^{p^{i-1}}-1$ em um fecho algébrico de $\mathbb{F}$ é também raiz de $p-\sum_{j=0}^{p-1} X^{j p^{i-1}}$ segue que $p-\sum_{j=0}^{p-1} X^{j p^{i-1}}=\left(X^{p^{i-1}}-1\right) h(X)$, para algum $h(X) \in \mathbb{F}[X]$.

E também, temos que

$$
\begin{aligned}
X^{p^{n}}-1 & =\left(X^{p^{i}}-1\right)\left(\sum_{j=0}^{p^{n-i}-1} X^{j p^{i}}\right) \\
& =\left(X^{p^{i-1}}-1\right)\left(\sum_{j=0}^{p-1} X^{j p^{i-1}}\right)\left(\sum_{j=0}^{p^{n-i}-1} X^{j p^{i}}\right) .
\end{aligned}
$$

Logo,

$m d c\left(e_{i}(X), X^{p^{n}}-1\right)=\left(X^{p^{i-1}}-1\right)\left(\sum_{j=0}^{p^{n-i}-1} X^{j p^{i}}\right) m d c\left(h(X), \sum_{j=0}^{p-1} X^{j p^{i-1}}\right)$.

Se $\operatorname{mdc}\left(h(X), \sum_{j=0}^{p-1} X^{j p^{i-1}}\right) \neq 1$ então existe uma raiz comum $\eta$ em alguma extensão algébrica de $\mathbb{F}$. Como $h(X) \mid\left(p-\sum_{j=0}^{p-1} X^{j p^{i-1}}\right)$ segue que $0=p-\sum_{j=0}^{p-1} \eta^{j p^{i-1}}=p$, uma contradição.

Assim, $\operatorname{mdc}\left(h(X), \sum_{j=0}^{p-1} X^{j p^{i-1}}\right)=1$ e portanto

$$
g_{i}(X)=\left(X^{p^{i-1}}-1\right) \sum_{j=0}^{p^{n-i}-1} X^{j p^{i}}, \quad 1 \leq i \leq n
$$


Para o idempotente $e_{0}$ segue que

$$
e_{0}=\widetilde{A}=\frac{1}{p^{n}} \sum_{j=0}^{p^{n}-1} X^{j}
$$

$\mathrm{e}$

$$
X^{p^{n}}-1=(X-1)\left(X^{p^{n}-1}+X^{p^{n}-2}+\cdots+X+1\right) .
$$

Logo, o polinômio gerador do ideal gerado pelo idempotente $e_{0}$ é

$$
g_{0}(X)=X^{p^{n}-1}+X^{p^{n}-2}+\cdots+X+1 .
$$

Agora, como o grau $\left(g_{i}(X)\right)=p^{n}-p^{i}+p^{i-1}$, para $1 \leq i \leq n$, obtemos que

$$
\begin{aligned}
\operatorname{dim}\left(\mathcal{I}_{i}\right) & =p^{n}-\operatorname{grau}\left(g_{i}(X)\right)=p^{n}-\left(p^{n}-p^{i}+p^{i-1}\right) \\
& =p^{i}-p^{i-1}=p^{i-1}(p-1) \\
& =\phi\left(p^{i}\right) .
\end{aligned}
$$

e para o ideal $\mathcal{I}_{0}$ temos

$$
\operatorname{dim}\left(\mathcal{I}_{0}\right)=p^{n}-\left(p^{n}-1\right)=1
$$

Exemplo 6.2.1. Sejam $G=\langle a\rangle$ um grupo cíclico de ordem 125 e $\mathbb{F}$ o corpo finito com 127 elementos. Note que, 127 tem ordem $\phi\left(5^{3}\right)=100$ módulo 125 .

Seja

$$
G=\langle a\rangle \supset\left\langle a^{5}\right\rangle \supset\left\langle a^{25}\right\rangle \supset\left\langle a^{125}\right\rangle=\{1\}
$$

a cadeia descendente todos os subgrupos cíclicos de $G$.

Então, segue, do Teorema 6.1.5, que o conjunto dos idempotentes primitivos de $\mathbb{F} G$ é dado por:

$$
e_{0}=\frac{1}{125}\left(\sum_{h \in G} h\right)
$$

$\mathrm{e}$

$$
e_{i}=\frac{1}{\left|\left\langle a^{5^{i}}\right\rangle\right|} \sum_{h \in\left\langle a^{5^{2}}\right\rangle} h-\frac{1}{\left|\left\langle a^{5^{i-1}}\right\rangle\right|} \sum_{h \in\left\langle a^{5^{i-1}}\right\rangle} h, \quad \text { para } 1 \leq i \leq 3 .
$$

Temos que, os subgrupos cíclicos de $G$ são:

$$
\begin{aligned}
\left\langle a^{5}\right\rangle= & \left\{1, a^{5}, a^{10}, a^{15}, a^{20}, a^{25}, a^{30}, a^{35}, a^{40}, a^{45}, a^{50}, a^{55}, a^{60}, a^{65}, a^{70}, a^{75},\right. \\
& \left.a^{80}, a^{85}, a^{90}, a^{95}, a^{100}, a^{105}, a^{100}, a^{115}, a^{120}\right\} \\
\left\langle a^{25}\right\rangle= & \left\{1, a^{25}, a^{50}, a^{75}, a^{100}\right\} \\
\left\langle a^{125}\right\rangle= & \{1\} .
\end{aligned}
$$


Vamos listar os idempotentes primitivos de $\mathbb{F} G$ :

$$
\begin{aligned}
e_{0} & =\frac{1}{125}\left(1+a+a^{2}+a^{3}+\cdots+a^{12-1}\right) ; \\
e_{1} & =\frac{1}{25}\left(1+a^{5}+a^{10}+a^{15}+\cdots+a^{120}\right)-\frac{1}{125}\left(1+a+a^{2}+a^{3}+\cdots+a^{124}\right) \\
& =\frac{1}{125}\left(5+5 a^{5}+5 a^{10}+\cdots+5 a^{120}-1-a-a^{2}-\cdots-a^{124}\right) \\
& =\frac{1}{125}\left(4-a-a^{2}-a^{3}-a^{4}+4 a^{5}-\cdots+4 a^{120}-a^{121}-a^{122}-a^{123}-a^{124}\right) ; \\
e_{2} & =\frac{1}{5}\left(1+a^{25}+a^{50}+a^{75}+a^{100}\right)-\frac{1}{25}\left(1+a^{5}+a^{10}+a^{15}+\cdots+a^{120}\right) \\
& =\frac{1}{25}\left(5+5 a^{25}+5 a^{50}+5 a^{75}+5 a^{100}-1-a^{5}-a^{10}-a^{15}-\cdots-a^{120}\right) \\
& =\frac{1}{25}\left(4-a^{5}-a^{10}-a^{15}-a^{20}+4 a^{25}-\cdots+4 a^{100}-a^{105}-a^{110}-a^{115}-a^{120}\right) ; \\
e_{3} & =1-\frac{1}{5}\left(1+a^{25}+a^{50}+a^{75}+a^{100}\right) \\
& =\frac{1}{5}\left(5-1-a^{25}-a^{50}-a^{75}-a^{100}\right) \\
& =\frac{1}{5}\left(4-a^{25}-a^{50}-a^{75}-a^{100}\right) ;
\end{aligned}
$$

Agora, vamos aplicar as fórmulas (1) e (2) para encontrarmos os geradores dos códigos cíclicos minimais de comprimento 125 sobre $\mathbb{F}$.

Assim, obtemos:

$$
\begin{aligned}
g_{0}= & 1+X+X^{2}+X^{3}+\cdots+X^{124} \\
g_{1}= & (X-1)\left(\sum_{j=0}^{24} X^{j 5}\right) \\
= & (X-1)\left(1+X^{5}+X^{10}+X^{15}+\cdots+X^{120}\right) \\
= & X+X^{6}+X^{11}+X^{16}+\cdots+X^{121}-1-X^{5}-X^{10}+ \\
& -X^{15}-\cdots-X^{120} \\
g_{2}= & \left(X^{5}-1\right)\left(\sum_{j=0}^{4} X^{j 25}\right) \\
= & \left(X^{5}-1\right)\left(1+X^{25}+X^{50}+X^{75}+X^{100}\right) \\
= & X^{5}+X^{30}+X^{55}+X^{80}+X^{105}-1-X^{25}-X^{50}-X^{75}-X^{100} ; \\
g_{3}= & X^{25}-1
\end{aligned}
$$

Os códigos $\mathcal{I}_{0}, \mathcal{I}_{1}, \mathcal{I}_{2}, \mathcal{I}_{3}$ cujos polinômios geradores são $g_{0}, g_{1}, g_{2} . g_{3}$ respectivamente são cíclicos de comprimento 125 com paràmetros: 


\begin{tabular}{|l||l||l||l|}
\hline Código & Dimensão & Polinômio Gerador & Idlempotente Gerador \\
\hline $\mathcal{I}_{0}$ & 1 & $g_{0}$ & $e_{0}$ \\
\hline $\mathcal{I}_{1}$ & 4 & $g_{1}$ & $e_{1}$ \\
\hline $\mathcal{I}_{2}$ & 20 & $g_{2}$ & $e_{2}$ \\
\hline $\mathcal{I}_{3}$ & 100 & $g_{3}$ & $e_{3}$ \\
\hline
\end{tabular}

\section{Geradores do código cíclico de comprimento $2 p^{n}$}

Nosso objetivo agora, é encontrar os polinômios geradores dos códigos cíclicos de comprimento $2 p^{n}$ cujos idempotentes geradores são dados pelo Teorema 6.1.6.

Assim, temos que

$$
\begin{aligned}
e_{i}(X) & =\frac{\left(1+X^{p^{n}}\right)}{2} \frac{1}{p^{n-i+1}}\left[p \sum_{j=0}^{p^{n-i}-1} X^{2 j p^{i}}-\sum_{j=0}^{p^{n-i+1}-1} X^{2 j p^{i-1}}\right] \\
& =\frac{\left(1+X^{p^{n}}\right)}{2} \frac{1}{p^{n-i+1}}\left[\left(p-\sum_{j=0}^{p-1} X^{2 j p^{i-1}}\right)\left(\sum_{j=0}^{p^{n-i}-1} X^{2 j p^{i}}\right)\right] .
\end{aligned}
$$

Como toda raiz do polinômio $1-X^{p^{i-1}}$ em um fecho algébrico de $\mathbb{F}$ é também raiz de $p-\sum_{j=0}^{p-1} X^{2 j p^{i-1}}$ segue que $p-\sum_{j=0}^{p-1} X^{2 j p^{i-1}}=\left(1-X^{p^{i-1}}\right) h(X)$, para algum $h(X) \in \mathbb{F}[X]$.

E também temos que

$$
\begin{aligned}
1-X^{2 p^{n}} & =\left(1-X^{p^{i}}\right)\left(1+X^{p^{i}}+\cdots+\left(X^{p^{i}}\right)^{2 p^{n-i}-1}\right) \\
& =\left(1-X^{p^{i-1}}\right)\left(1+X^{p^{i-1}}+\cdots+\left(X^{p^{i-1}}\right)^{p-1}\right)\left(1+X^{p^{i}}+\cdots+\left(X^{p^{i}}\right)^{2 p^{n-i}-1}\right) .
\end{aligned}
$$

Como $1+X^{p^{i}}+\cdots+\left(X^{p^{i}}\right)^{2 p^{n-i}-1}=\sum_{j=0}^{p^{n-i}-1} X^{2 j p^{i}}+X^{p^{i}} \sum_{j=0}^{p^{n-i}-1} X^{2 j p^{i}}$ temos que

$$
\begin{aligned}
1-X^{2 p^{n}} & =\left(1-X^{p^{i-1}}\right)\left(\sum_{j=0}^{p-1} X^{j p^{i-1}}\right)\left(\sum_{j=0}^{p^{n-i}-1} X^{2 j p^{2}}+X^{p^{i}} \sum_{j=0}^{p^{n-i}-1} X^{2 j p^{i}}\right) \\
& \left.=\left(1-X^{p^{i-1}}\right)\left(\sum_{j=0}^{p-1} X^{j p^{i-1}}\right)\left(1+X^{p^{2}}\right) \sum_{j=0}^{p^{n-i}-1} X^{2 j p^{i}}\right) \\
& =\left(1-X^{p^{i-1}}\right)\left(1+X^{p^{2}}\right)\left(\sum_{j=1}^{p-1} X^{j p^{i-1}}\right)\left(\sum_{j=0}^{p^{n-2}-1} X^{2 j p^{i}}\right) .
\end{aligned}
$$


Consideremos o polinômio $d_{i}(X)=\left(1-X^{p^{i-1}}\right)\left(1+X^{p^{i}}\right)\left(\sum_{j=0}^{p^{n-i}-1} X^{2 j p^{i}}\right)$ que é um divisor comum dos polinômios $1-X^{2 p^{n}}$ e $e_{i}(X)$. Note que o grau do polinômio $d_{i}(X)$ é igual a $2 p^{n}-p^{i}+p^{i-1}$.

Seja $g_{i}(X)=\operatorname{mdc}\left(e_{i}(X), 1-X^{2 p^{n}}\right)$. Então $d_{i} \mid g_{i}$.

Seja ainda $\mathcal{I}_{i}$ o ideal gerado pelo polinômio $g_{i}$. Como já mostramos que $\operatorname{dim}\left(\mathcal{I}_{i}\right)=\phi\left(p^{i}\right)$ temos que $\operatorname{grau}\left(g_{i}(X)\right)=2 p^{n}-\phi\left(p^{i}\right)=2 p^{n}-p^{i}+p^{i-1}=$ $\operatorname{grau}\left(d_{i}\right)$.

Consequentemente, tem-se que $d_{i}=g_{i}$.

Portanto,

$$
g_{i}(X)=\left(1-X^{p^{i-1}}\right)\left(1+X^{p^{i}}\right) \sum_{j=0}^{p^{n-i}-1} X^{2 j p^{i}}, \quad \text { para } \quad 1 \leq i \leq n .
$$

Agora, consideremos o idempotente

$$
e_{i}^{*}(X)=\frac{\left(1-X^{p^{n}}\right)}{2} \frac{1}{p^{n-i+1}}\left[\left(p-\sum_{j=0}^{p-1} X^{2 j p^{i-1}}\right)\left(\sum_{j=0}^{p^{n-i}-1} X^{2 j p^{i}}\right)\right] .
$$

E como toda raiz do polinômio $1+X^{p^{i-1}}$ em um fecho algébrico de $\mathbb{F}$ é também raiz de $p-\sum_{j=0}^{p-1} X^{2 j p^{i-1}}$ segue que $p-\sum_{j=0}^{p-1} X^{2 j p^{i-1}}=(1+$ $\left.X^{p^{i-1}}\right) t(X)$, para algum $t(X) \in \mathbb{F}[X]$.

Temos também que,

$$
\begin{aligned}
1-X^{2 p^{n}}= & \left(1+X^{p^{i}}\right)\left(1-X^{p^{i}}+X^{2 p^{i}}-X^{3 p^{i}}+\cdots+(-1)^{2 p^{n-i}-1}\left(X^{p^{i}}\right)^{2 p^{n-i}-1}\right) \\
= & \left(1+X^{p^{i-1}}\right)\left(1-X^{p^{i-1}}+X^{2 p^{i-1}}+\cdots+(-1)^{p-1}\left(X^{p^{i-1}}\right)^{p-1}\right)\left(1-X^{p^{i}}+X^{2 p^{i}}+\right. \\
& \left.-X^{3 p^{i}}+\cdots+(-1)^{2 p^{n-i}-1}\left(X^{p^{i}}\right)^{2 p^{n-i}-1}\right) .
\end{aligned}
$$

Como $1-X^{p^{i}}+X^{2 p^{i}}-X^{3 p^{i}}+\cdots+(-1)^{2 p^{n-i}-1}\left(X^{p^{i}}\right)^{2 p^{n-i}-1}=\left(\sum_{j=0}^{p^{n-i}-1} X^{2 j p^{i}}-X^{p^{i}} \sum_{j=0}^{p^{n-i}-1} X^{2 j p^{i}}\right)$ temos que

$$
\begin{aligned}
1-X^{-2 p^{n}} & =\left(1+X^{p^{i-1}}\right)\left(\sum_{j=0}^{p-1}(-X)^{j p^{i-1}}\right)\left(\sum_{j=0}^{p^{n-i}-1} X^{2 j p^{2}}-X^{p^{i}} \sum_{j=0}^{p^{n-i}-1} X^{2 j p^{i}}\right) \\
& =\left(1+X^{p^{i-1}}\right)\left(\sum_{j=0}^{p-1}(-X)^{j p^{i-1}}\right)\left(\left(1-X^{p^{i}}\right) \sum_{j=0}^{p^{n-1}-1} X^{-2 j p^{2}}\right) \\
& =\left(1+X^{p^{i-1}}\right)\left(1-X^{p^{i}}\right)\left(\sum_{j=0}^{p-1}(-X)^{j p^{i-1}}\right)\left(\sum_{j=0}^{p^{n-i}-1} X^{-2 j p^{i}}\right) .
\end{aligned}
$$


Consideremos o polinômio $d_{i}(X)=\left(1+X^{p^{i-1}}\right)\left(1-X^{p^{i}}\right)\left(\sum_{j=0}^{p^{n-i}-1} X^{2 j p^{i}}\right)$ que é um divisor comum dos polinômios $1-X^{2 p^{n}}$ e $e_{i}^{*}(X)$. Note que o grau do polinômio $d_{i}(X)$ é igual a $2 p^{n}-p^{i}+p^{i-1}$.

Seja $g_{i}^{*}(X)=\operatorname{mdc}\left(e_{i}^{*}(X), 1-X^{2 p^{n}}\right)$. Então $d_{i} \mid g_{i}^{*}$.

Seja ainda $\mathcal{J}_{i}$ o ideal gerado pelo polinômio $g_{i}^{*}$. Como já mostramos que $\operatorname{dim}\left(\mathcal{J}_{i}\right)=\phi\left(p^{i}\right)$ temos que $\operatorname{grau}\left(g_{i}^{*}(X)\right)=2 p^{n}-\phi\left(p^{i}\right)=2 p^{n}-p^{i}+p^{i-1}=$ $\operatorname{grau}\left(d_{i}\right)$.

Consequentemente, tem-se que $d_{i}=g_{i}^{*}$.

Portanto,

$g_{i}^{*}(X)=\left(1+X^{p^{i-1}}\right)\left(1-X^{p^{i}}\right) \sum_{j=0}^{p^{n-i}-1} X^{j 2 p^{i}}, \quad$ para $\quad 1 \leq i \leq n$.

Para os idempotentes

$$
e_{0}=\frac{\left(1+X^{p^{n}}\right)}{2} \frac{1}{p^{n}}\left(\sum_{h \in A} h\right)=\frac{\left(1+X^{p^{n}}\right)}{2 p^{n}}\left(\sum_{j=0}^{p^{n}-1} X^{2 j}\right)
$$

$\mathrm{e}$

$$
e_{0}^{*}=\frac{\left(1-X^{p^{n}}\right)}{2 p^{n}}\left(\sum_{j=0}^{p^{n}-1} X^{2 j}\right)
$$

Temos que

$$
g_{0}(X)=(1+X)\left(\sum_{j=0}^{p^{n}-1} X^{2 j}\right)
$$

e

$$
g_{0}^{*}(X)=(1-X)\left(\sum_{j=0}^{p^{n}-1} X^{2 j}\right)
$$

Exemplo 6.2.2. Sejam $G=\langle a\rangle$ um grupo cíclico de ordem 54 e $\mathbb{F}$ o corpo finito com 59 elementos. Note que, 59 tem ordem $\phi\left(3^{3}\right)=18$ módulo 54 .

Escrevendo $G=A \times C$, oncle $A$ é o 3 -subgrupo de Sylow de $G$ e $C=\left\{1, a^{27}\right\}$ é o seu 2-subgrupo de Sylow. 
Seja

$$
A=\left\langle a^{2}\right\rangle \supset\left\langle a^{6}\right\rangle \supset\left\langle a^{18}\right\rangle \supset\left\langle a^{54}\right\rangle=\{1\}
$$

a cadeia descendente de todos os subgrupos cíclicos de $A$.

Assim, temos que os subgrupos cíclicos de $A$ são:

$$
\begin{aligned}
\left\langle a^{2}\right\rangle= & \left\{1, a^{2}, a^{4}, a^{6}, a^{8}, a^{10}, a^{12}, a^{14}, a^{16}, a^{18}, a^{20}, a^{22}, a^{24}, a^{26}, a^{28}, a^{30},\right. \\
& \left.a^{32}, a^{34}, a^{36}, a^{38}, a^{10}, a^{42}, a^{44}, a^{46}, a^{48}, a^{50}, a^{52}\right\} \\
\left\langle a^{6}\right\rangle= & \left\{1, a^{6}, a^{12}, a^{18}, a^{24}, a^{30}, a^{36}, a^{42}, a^{48}\right\} \\
\left\langle a^{18}\right\rangle= & \left\{1, a^{18}, a^{36}\right\} \\
\left\langle a^{54}\right\rangle= & \{1\} .
\end{aligned}
$$

Segue, do Teorema 6.1.6, que o conjunto dos idempotentes primitivos de $\mathbb{F} G$ é dado por:

$$
\varepsilon_{i}=\frac{\left(1+a^{27}\right)}{2} e_{i} \quad \text { e } \quad \varepsilon_{i}^{\prime}=\frac{\left(1-a^{27}\right)}{2} e_{i}, \quad 0 \leq i \leq 3
$$

onde os elementos $e_{i}, 1 \leq i \leq 3$, são os idempotentes primitivos de $\mathbb{F} A$, ou seja, $e_{0}=\widetilde{A}$ e $e_{i}=\widetilde{A}_{i}-\widetilde{A}_{i-1}$.

Calculando estes idempotentes obtemos,

$$
\begin{aligned}
\varepsilon_{0}= & \frac{\left(1+X^{27}\right)}{2 \times 27}\left(\sum_{j=0}^{26} X^{2 j}\right)=\frac{1}{54}\left(\sum_{j=0}^{26} X^{2 j}+X^{27} \sum_{j=0}^{26} X^{2 j}\right) \\
= & \frac{1}{54}\left(1+X+X^{2}+X^{3}+\cdots+X^{53}\right) ; \\
\varepsilon_{0}^{\prime}= & \frac{\left(1-X^{27}\right)}{2 \times 27}\left(\sum_{j=0}^{26} X^{2 j}\right) \\
= & \frac{1}{54}\left(1-X+X^{2}-X^{3}+\cdots+X^{52}-X^{53}\right) ; \\
\varepsilon_{1}= & \frac{\left(1+X^{27}\right)}{2}\left(\frac{1}{9} \widehat{\left\langle a^{6}\right\rangle}-\frac{1}{27} \widehat{\left\langle a^{2}\right\rangle}\right) \\
= & \frac{\left(1+X^{27}\right)}{54}\left(3 \widehat{\left\langle a^{6}\right\rangle}-\widehat{\left\langle a^{2}\right\rangle}\right) \\
= & \frac{1}{54}\left[2 \left(1+X^{6}+X^{12}+X^{18}+X^{24}+X^{30}+X^{36}+X^{42}+X^{48}+X^{3}+X^{9}+\right.\right. \\
& \left.+X^{15}+X^{21}+X^{27}+X^{33}+X^{39}+X^{45}+X^{51}\right)-\left(X^{2}+X^{4}+X^{8}+X^{10}+\right. \\
& +X^{14}+X^{16}+X^{20}+X^{22}+X^{26}+X^{28}+X^{32}+X^{34}+X^{38}+X^{40}+X^{44}+ \\
& +X^{46}+X^{50}+X^{52}+X+X^{5}+X^{7}+X^{11}+X^{13}+X^{17}+X^{19}+X^{23}+ \\
& \left.\left.+X^{25}+X^{29}+X^{31}+X^{35}+X^{37}+X^{41}+X^{43}+X^{47}+X^{49}+X^{53}\right)\right] ;
\end{aligned}
$$




$$
\begin{aligned}
& \varepsilon_{1}^{\prime}=\frac{\left(1-X^{27}\right)}{2}\left(\frac{1}{9} \widehat{\left\langle a^{6}\right\rangle}-\frac{1}{27} \widehat{\left\langle a^{2}\right\rangle}\right) \\
& =\frac{\left(1-X^{27}\right)}{54}\left(3 \widehat{\left\langle a^{6}\right\rangle}-\widehat{\left\langle a^{2}\right\rangle}\right) \\
& =\frac{1}{54}\left[2 \left(1+X^{6}+X^{12}+X^{18}+X^{24}+X^{30}+X^{36}+X^{42}+X^{48}-X^{3}-X^{9}+\right.\right. \\
& \left.-X^{15}-X^{21}-X^{27}-X^{33}-X^{39}-X^{45}-X^{51}\right)-\left(X^{2}+X^{4}+X^{8}+X^{10}+\right. \\
& +X^{14}+X^{16}+X^{20}+X^{22}+X^{26}+X^{28}+X^{32}+X^{34}+X^{38}+X^{40}+X^{44}+ \\
& +X^{46}+X^{50}+X^{52}-X-X^{5}-X^{7}-X^{11}-X^{13}-X^{17}-X^{19}-X^{23}+ \\
& \left.\left.-X^{25}-X^{29}-X^{31}-X^{35}-X^{37}-X^{41}-X^{43}-X^{47}-X^{49}-X^{53}\right)\right] \text {; } \\
& \varepsilon_{2}=\frac{\left(1+X^{27}\right)}{2}\left(\frac{1}{3} \widehat{\left\langle a^{18}\right\rangle}-\frac{1}{9} \widehat{\left\langle a^{6}\right\rangle}\right) \\
& =\frac{\left(1+X^{27}\right)}{18}\left(\widehat{\left.3 a^{18}\right\rangle}-\widehat{\left\langle a^{6}\right\rangle}\right) \\
& =\frac{1}{18}\left[\left(2\left(1+X^{18}+X^{36}+X^{9}+X^{27}+X^{45}\right)-\left(X^{6}+X^{12}+X^{24}+X^{30}+\right.\right.\right. \\
& \left.\left.+X^{42}+X^{48}+X^{3}+X^{15}+X^{21}+X^{33}+X^{39}+X^{51}\right)\right] \text {; } \\
& \varepsilon_{2}^{\prime}=\frac{\left(1-X^{27}\right)}{2}\left(\frac{1}{3} \widehat{\left\langle a^{18}\right\rangle}-\frac{1}{9} \widehat{\left\langle a^{6}\right\rangle}\right) \\
& =\frac{\left(1-X^{27}\right)}{18}\left(3 \widehat{\left\langle a^{18}\right\rangle}-\widehat{\left\langle a^{6}\right\rangle}\right) \\
& \begin{aligned}
= & \frac{1}{18}\left[\left(2\left(1+X^{18}+X^{36}-X^{9}-X^{27}-X^{45}\right)+\left(-X^{6}-X^{12}-X^{24}-X^{30}+\right.\right.\right. \\
& \left.\left.-X^{42}-X^{48}+X^{3}+X^{15}+X^{21}+X^{33}+X^{39}+X^{51}\right)\right] ;
\end{aligned} \\
& \varepsilon_{3}=\frac{\left(1+X^{27}\right)}{2}\left(\widehat{\left\langle a^{54}\right\rangle}-\frac{1}{3} \widehat{\left\langle a^{18}\right\rangle}\right) \\
& =\frac{\left(1+X^{27}\right)}{6}\left(3 \widehat{\left\langle a^{18}\right\rangle}-\widehat{\left\langle a^{6}\right\rangle}\right) \\
& =\frac{1}{6}\left[\left(2\left(1+X^{27}\right)-\left(X^{9}+X^{18}+X^{36}+X^{45}\right)\right]\right. \text {; } \\
& \varepsilon_{3}^{\prime}=\frac{\left(1-X^{27}\right)}{2}\left(\widehat{\left\langle a^{54}\right\rangle}-\frac{1}{3} \widehat{\left\langle a^{18}\right\rangle}\right) \\
& =\frac{\left(1-X^{27}\right)}{6}\left(3 \widehat{\left\langle a^{54}\right\rangle}-\widehat{\left\langle a^{18}\right\rangle}\right) \\
& =\frac{1}{6}\left[\left(2\left(1-X^{2 \tau}\right)+\left(X^{9}-X^{18}-X^{36}+X^{15}\right)\right]\right. \text {; }
\end{aligned}
$$

Agora, vamos aplicar as fórmulas (3), (4), (5) e (6) para encontrarmos os gera- 
dores $g_{i}(X)$ e listarmos os códigos cíclicos minimais cle comprimento 54 sobre $\mathbb{F}$.

Assim, obtemos:

$$
\begin{aligned}
g_{0}= & (1+X)\left(\sum_{j=0}^{26} X^{2 j}\right) \\
= & 1+X+X^{2}+X^{3}+\cdots+X^{53} \\
g_{0}^{\prime}= & (1-X)\left(\sum_{j=0}^{26} X^{2 j}\right) \\
= & 1-X+X^{2}-X^{3}+X^{4}+\cdots+X^{52}-X^{53} \\
g_{1}= & (1-X)\left(1+X^{3}\right)\left(\sum_{j=0}^{8} X^{2 j 3}\right) \\
= & (1-X)\left(1+X^{3}\right)\left(1+X^{6}+X^{12}+X^{18}+X^{24}+X^{30}+X^{36}+X^{42}+X^{48}\right) \\
= & (1-X)\left(1+X^{6}+X^{12}+X^{18}+X^{24}+X^{30}+X^{36}+X^{42}+\right. \\
& \left.+X^{48}+X^{27}+X^{33}+X^{39}+X^{45}+X^{51}+X^{3}+X^{9}+X^{15}+X^{21}\right) ; \\
g_{1}^{\prime}= & (1+X)\left(1-X^{3}\right)\left(\sum_{j=0}^{8} X^{2 j 3}\right) \\
= & (1+X)\left(1-X^{3}\right)\left(1+X^{6}+X^{12}+X^{18}+X^{24}+X^{30}+X^{36}+X^{42}+X^{48}\right) \\
= & (1+X)\left(1+X^{6}+X^{12}+X^{18}+X^{24}+X^{30}+X^{36}+X^{42}+\right. \\
& \left.+X^{48}-X^{27}-X^{33}-X^{39}-X^{45}-X^{51}-X^{3}-X^{9}-X^{15}-X^{21}\right) ; \\
g_{3}= & \left(1-X^{9}\right)\left(1+X^{27}\right) ; \\
g_{2}= & \left(1-X^{3}\right)\left(1+X^{9}\right)\left(\sum_{j=0}^{2} X^{2 j 9}\right) \\
= & \left(1-X^{3}\right)\left(1+X^{9}\right)\left(1+X^{18}+X^{36}\right) \\
= & \left(1-X^{3}\right)\left(1+X^{18}+X^{36}+X^{27}+X^{45}+X^{9}\right) ; \\
g_{2}^{\prime} & \left(1+X^{3}\right)\left(1-X^{9}\right)\left(\sum_{j=0}^{2} X^{2 j 9}\right) \\
= & \left(1+X^{3}\right)\left(1-X^{9}\right)\left(1+X^{18}+X^{36}\right) \\
= & \left(1+X^{3}\right)\left(1+X^{18}+X^{36}-X^{27}-X^{45}-X^{9}\right) ; \\
= & \\
= & \\
= & \\
= & \\
= &
\end{aligned}
$$




$$
g_{3}^{\prime}=\left(1+X^{9}\right)\left(1-X^{27}\right)
$$

Os códigos cíclicos $\mathcal{I}_{0}, \mathcal{I}_{1}, \mathcal{I}_{2}, \mathcal{I}_{3}$ gerados respectivamente por $g_{0}, g_{1}, g_{2}, g_{3}$ e os códigos cíclicos $\mathcal{J}_{0}, \mathcal{J}_{1}, \mathcal{J}_{2}, \mathcal{J}_{3}$ gerados respectivamente por $g_{0}^{\prime}, g_{1}^{\prime}, g_{2}^{\prime}, g_{3}^{\prime}$ todos de comprimento 54 têm como parâmetros:

\begin{tabular}{|l||l||l||l|}
\hline Código & Dimensão & Polinômio Gerador & Idempotente Gerador \\
\hline $\mathcal{I}_{0}$ & 1 & $g_{0}$ & $e_{0}$ \\
\hline $\mathcal{J}_{0}$ & 1 & $g_{0}^{\prime}$ & $e_{0}^{\prime}$ \\
\hline $\mathcal{I}_{1}$ & 2 & $g_{1}$ & $e_{1}$ \\
\hline $\mathcal{J}_{1}$ & 2 & $g_{1}^{\prime}$ & $e_{1}^{\prime}$ \\
\hline $\mathcal{I}_{2}$ & 6 & $g_{2}$ & $e_{2}$ \\
\hline $\mathcal{J}_{2}$ & 6 & $g_{2}^{\prime}$ & $e_{2}^{\prime}$ \\
\hline $\mathcal{I}_{3}$ & 18 & $g_{3}$ & $e_{3}$ \\
\hline $\mathcal{J}_{3}$ & 18 & $g_{3}^{\prime}$ & $e_{3}^{\prime}$ \\
\hline
\end{tabular}




\subsection{Apêndice}

Lembramos que, clado um grupo abeliano $A$, a classe ciclotômica de um elemento $h \in A$, é o conjunto

$$
\mathcal{S}_{h}=\left\{h^{q^{j}} \mid 0 \leq j \leq t_{h}-1\right\}
$$

onde $t_{h}$ é o menor inteiro positivo tal que

$$
q^{t_{h}} \equiv 1 \quad(\bmod o(h))
$$

e a classe ciclotômica de um inteiro $s$ módulo $n$ é

$$
\Omega_{s}=\left\{\bar{s}, \overline{s q}, \ldots \overline{s q^{r_{s}-1}}\right\}
$$

onde $\overrightarrow{s q^{i}}$ é a classe de $s q^{i}$ módulo $n$ e $r_{s}$ é o menor inteiro positivo tal que

$$
s q^{r_{s}} \equiv s \quad(\bmod n)
$$

Considere $G=\langle a\rangle$ o grupo cíclico de ordem $m$.

Assim, para todo $h \in G$ tem-se que $h=a^{s}$, para algum inteiro $s$.

Logo,

$$
\mathcal{S}_{a^{s}}=\left\{a^{s q^{j}} \mid 0 \leq j \leq r_{s}-1\right\}=\left\{a^{l} \mid l \in \Omega_{s}\right\} .
$$

Vamos considerar inicialmente o caso em que $m=p^{n}$, para algum primo $p$.

Lema 6.3.1. ([19], Lema 3.1) Seja q uma potência de primo e suponhamos que q tem ordem $\phi\left(p^{n}\right)$ módulo $p^{n}$. Então existem $n$ q-classes ciclotômicas módulo $p^{n}$, distintas, não nulas, dadas por

$$
\Omega_{j}=\left\{p^{j-1}, p^{j-1} q, \ldots, p^{j-1} q^{\phi\left(p^{n-j+1}\right)-1}\right\},
$$

para $1 \leq j \leq n$.

Demonstração: Suponhamos que $q$ tem ordem $\phi\left(p^{n}\right)$ módulo $p^{n}$, isto é, que $\bar{q}$ gera $\mathbb{Z}_{p^{n}}$.

Para $1 \leq j \leq n$, tem-se que $p^{n-j+1} \mid p^{n}$.

Considere o epimorfismo natural de $\mathbb{Z}_{p^{n}}$ em $\mathbb{Z}_{p^{n-\jmath+1}}$. Assim, se $\bar{q} \in \mathbb{Z}_{p^{n}}$ é un gerador do grupo cíclico $U\left(\mathbb{Z}_{p^{n}}\right)$ tem-se que $\bar{q} \in \mathbb{Z}_{p^{n-1+1}}$ é um gerador 
do grupo cíclico $U\left(\mathbb{Z}_{p^{n-j+1}}\right)$, donde $q$ tem ordem $\phi\left(p^{n-j+1}\right)$ módulo $p^{n-j+1}$. Então,

$$
q^{\phi\left(p^{n-j+1}\right)} \equiv 1 \quad\left(\bmod p^{n-j+1}\right), \quad 1 \leq j \leq n,
$$

ou equivalentemente,

$$
p^{j-1} q^{\phi\left(p^{n-j+1}\right)} \equiv p^{j-1} \quad\left(\bmod p^{n}\right) .
$$

Portanto,

$$
\Omega_{j}=\left\{p^{j-1}, p^{j-1} q, \ldots, p^{j-1} q^{\phi\left(p^{n-j+1}\right)-1}\right\},
$$

são $q$-classes ciclotômicas módulo $p^{n}$, não nulas, e distintas.

Note que, cada conjunto $\Omega_{j}$ tem $\phi\left(p^{n-j+1}\right)$ elementos. Então,

$$
\begin{aligned}
\sum_{j=1}^{n} \phi\left(p^{j}\right) & =\phi(p)+\phi\left(p^{2}\right)+\cdots+\phi\left(p^{n}\right) \\
& =p-1+p(p-1)+p^{2}(p-1)+\cdots+p^{n-1}(p-1) \\
& =p-1+p^{2}-p+p^{3}-p^{2}+\cdots+p^{n}-p^{n-1} \\
& =p^{n}-1 .
\end{aligned}
$$

Como os conjuntos $\Omega_{i}$ e $\Omega_{j}$ são disjuntos dois a dois e o número de elementos da união de todos os $\Omega_{i}$ é igual a $p^{n}-1$. Segue que $\Omega_{1}, \Omega_{2}, \ldots, \Omega_{n}$ são todas as $q$-classes ciclotômicas módulo $p^{n}$ distintas e não nulas.

Seja $A$ um grupo cíclico de ordem $p^{n}$. Definimos $\overline{\mathcal{C}}_{i}=\sum_{s \in \Omega_{i}} a^{s} \in \mathbb{F} A$, para $1 \leq i \leq n$. isto é,

Lembremos que as classes ciclotômicas particionam os inteiros módulo $p^{n}$,

$$
\left\{\overline{0}, \overline{1}, \ldots, \overline{p^{n}-1}\right\}=\bigcup_{s} \Omega_{s}
$$

onde $s$ percorre um sistema de representantes para as classes ciclotômicas módulo $p^{n}$.

Vamos mostrar que os idempotentes primitivos que encontramos no Teorema 6.1.5 são os mesmos encontrados por Pruthi e Arora ([19], Teorema 3.5) que são os seguintes:

$$
e_{0}=\frac{1}{p^{n}}\left(1+\sum_{i=1}^{n} \overline{\mathcal{C}}_{i}\right)
$$


e

$$
e_{i}=\frac{1}{p^{n-i+1}}\left[(p-1)\left(1+\overline{\mathcal{C}}_{i+1}+\cdots+\overline{\mathcal{C}}_{n}\right)-\overline{\mathcal{C}}_{i}\right], \quad \text { para } \quad 1 \leq i \leq n .
$$

Reescrevendo o idempotente $e_{0}$ temos:

$$
\begin{aligned}
e_{0} & =\frac{1}{p^{n}}\left(1+\overline{\mathcal{C}}_{1}+\overline{\mathcal{C}}_{2}+\cdots+\overline{\mathcal{C}}_{n}\right) \\
& =\frac{1}{p^{n}}\left(1+\sum_{s \in \Omega_{1}} a^{s}+\sum_{s \in \Omega_{2}} a^{s}+\cdots+\sum_{s \in \Omega_{n}} a^{s}\right) .
\end{aligned}
$$

Logo, segue da observação acima, que

$$
e_{0}=\frac{1}{p^{n}} \sum_{a \in A} a \text {. }
$$

Portanto, o idempotente $e_{0}$ é o mesmo que nós encontramos.

Agora verificaremos os demais elementos idempotentes $e_{i}$.

Para $1 \leq i \leq n$, temos

$$
\begin{aligned}
e_{i} & =\frac{1}{p^{n-i+1}}\left[(p-1)\left(1+\overline{\mathcal{C}}_{i+1}+\cdots+\overline{\mathcal{C}}_{n}\right)-\overline{\mathcal{C}}_{i}\right] \\
& =\frac{1}{p^{n-i+1}}\left[p\left(1+\overline{\mathcal{C}}_{i+1}+\cdots+\overline{\mathcal{C}}_{n}\right)-\left(1+\overline{\mathcal{C}}_{i}+\overline{\mathcal{C}}_{i+1}+\cdots+\overline{\mathcal{C}}_{n}\right)\right] \\
& =\frac{1}{p^{n-i}}\left(1+\overline{\mathcal{C}}_{i+1}+\cdots+\overline{\mathcal{C}}_{n}\right)-\frac{1}{p^{n-i+1}}\left(1+\overline{\mathcal{C}}_{i}+\overline{\mathcal{C}}_{i+1}+\cdots+\overline{\mathcal{C}}_{n}\right)
\end{aligned}
$$

Assim, temos

$$
\begin{aligned}
& 1+\overline{\mathcal{C}}_{i+1}+\cdots+\overline{\mathcal{C}}_{n}=\left(1+\sum_{s \in \Omega_{i+1}} a^{s}+\sum_{s \in \Omega_{i+2}} a^{s}+\cdots+\sum_{s \in \Omega_{n}} a^{s}\right) \\
& =1+\left(a^{p^{i}}+a^{p^{i} q}+\cdots+a^{p^{2} q^{\phi\left(p^{n-i}\right)-1}}\right)+\left(a^{p^{i+1}}+a^{p^{i+1} q}+\cdots+a^{p^{i+1} q^{\phi\left(p^{n-i-1}\right)-1}}\right)+ \\
& \cdots+\left(a^{p^{n-1}}+a^{p^{n-1} q}+\cdots+a^{p^{n-1} q^{\phi(p)-1}}\right) \\
& =1+\left(a^{p^{i}}+\left(a^{p^{i}}\right)^{q}+\cdots+\left(a^{p^{2}}\right)^{q^{\rho\left(p^{n-i}\right)-1}}\right)+\left(\left(a^{p^{i}}\right)^{p}+\left(a^{p^{i}}\right)^{p q}+\cdots+\left(a^{p^{i}}\right)^{p q^{\rho\left(p^{n-1-1}\right)-1}}\right)+ \\
& \cdots+\left(\left(a^{p^{i}}\right)^{p^{n-i-1}}+\left(a^{p^{i}}\right)^{p^{n-1-1}} q+\cdots+\left(a^{p^{i}}\right)^{p^{n-1-1}} q^{o(p)-1}\right)
\end{aligned}
$$


Agora, consideremos o subgrupo cíclico $A_{i}=\left\langle a^{p^{i}}\right\rangle$ onde $\left|A_{i}\right|=p^{n-i}$, ou seja, $A_{i}=\left\{1, a^{p^{i}}, a^{2 p^{i}}, \ldots,\left(a^{p^{i}}\right)^{p^{n-i}-1}\right\}$.

Uma partição dos inteiros módulo $p^{n-i}$ é a união das $n-i q$-classes ciclotômicas, dadas por

$$
\Omega_{j}=\left\{p^{j-1}, p^{j-1} q, \ldots, p^{j-1} q^{\phi\left(p^{n-i-j+1}\right)-1}\right\},
$$

para $1 \leq j \leq n-i$

Assim,

$$
\begin{aligned}
\Omega_{1} & =\left\{1, q, \ldots, q^{\phi\left(p^{n-i}\right)-1}\right\} \\
\Omega_{2} & =\left\{p, p q, \ldots, p q^{\phi\left(p^{n-i-1}\right)-1}\right\} \\
\vdots & =\vdots \\
\Omega_{n-i} & =\left\{p^{n-i-1}, p^{n-i-1} q, \ldots, p^{n-i-1} q^{\phi(p)-1}\right\} .
\end{aligned}
$$

Observe que os expoentes dos elementos da forma $a^{p^{i}}$ do segundo termo do segundo membro de $\left(^{*}\right)$ estão em $\Omega_{1}$, os expoentes do terceiro termo do segundo membro de $\left(^{*}\right)$ estão em $\Omega_{2}$ e assim sucessivamente até os expoentes do último termo de $(*)$ que estão em $\Omega_{n-i}$.

Logo,

$$
1+\overline{\mathcal{C}}_{i+1}+\cdots+\overline{\mathcal{C}}_{n}=\sum_{a \in A_{i}} a
$$

Analogamente, consideremos o subgrupo cíclico $A_{i-1}=\left\langle a^{p^{i-1}}\right\rangle$ onde $\left|A_{i-1}\right|=$ $p^{n-i+1}$, isto é, $A_{i-1}=\left\{1, a^{p^{i-1}}, a^{2 p^{i-1}}, \ldots,\left(a^{p^{i-1}}\right)^{p^{n-i+1}-1}\right\}$.

Assim, uma partição dos inteiros módulo $p^{n-i+1}$ é a união das $n-i+1$ $q$-classes ciclotômicas, dadas por

$$
\begin{aligned}
\Omega_{1} & =\left\{1, q, \ldots, q^{\phi\left(p^{n-i+1}\right)-1}\right\} \\
\Omega_{2} & =\left\{p, p q, \ldots, p q^{\phi\left(p^{n-i}\right)-1}\right\} \\
\vdots & =\vdots \\
\Omega_{n-i+1} & =\left\{p^{n-i}, p^{n-i} q, \ldots, p^{n-i} q^{\phi(p)-1}\right\} .
\end{aligned}
$$

e

$$
1+\overline{\mathcal{C}_{i}}+\overline{\mathcal{C}}_{i+1}+\cdots+\overline{\mathcal{C}}_{n}=\left(1+\sum_{s \in \Omega_{i}} a^{s}+\sum_{s \in \Omega_{i+1}} a^{s}+\cdots+\sum_{s \in \Omega_{n}} a^{s}\right)
$$




$$
\begin{aligned}
&=1+\left(a^{p^{i-1}}+a^{p^{i-1} q}++\cdots+a^{p^{i-1}} q^{\phi\left(p^{n-i+1}\right)-1}\right)+\left(a^{p^{i}}+a^{p^{i} q}+\cdots+a^{p^{i} q^{\phi\left(p^{n-i}\right)-1}}\right)+ \\
& \cdots+\left(a^{p^{p^{-1}}}+a^{p^{n-1} q}+\cdots+a^{p^{n-1}} q^{\phi(p)-1}\right) \\
&=1+\left(a^{p^{i-1}}+\left(a^{p^{i-1}}\right)^{q}+\cdots+\left(a^{p^{i-1}}\right)^{\left.q^{\phi\left(p^{n-i+1}\right.}\right)-1}\right)+\left(\left(a^{p^{i-1}}\right)^{p}+\left(a^{p^{i-1}}\right) p q+\cdots+\right. \\
&\left.\left(a^{p^{i-1}}\right)^{p q^{\phi\left(p^{n-i}\right)-1}}\right)+\cdots+\left(\left(a^{p^{i-1}}\right)^{p^{n-i}}+\left(a^{p^{i-1}}\right)^{p^{n-i} q}+\cdots+\left(a^{p^{i-1}}\right)^{p^{n-i}} q^{\phi(p)-1}\right)
\end{aligned}
$$

Note que os expoentes dos elementos da forma $a^{p^{i-1}}$ de cada termo do laclo direito da equação (**) pertencem as $n-i+1$ q-classes ciclotômicas módulo $p^{n-i+1}$ dadas acima.

Logo,

$$
1+\overline{\mathcal{C}}_{i}+\overline{\mathcal{C}}_{i+1}+\cdots+\overline{\mathcal{C}}_{n}=\sum_{a \in A_{i-1}} a
$$

Portanto,

$$
\begin{aligned}
e_{i} & =\frac{1}{p^{n-i}}\left(1+\overline{\mathcal{C}}_{i+1}+\cdots+\overline{\mathcal{C}}_{n}\right)-\frac{1}{p^{n-i+1}}\left(1+\overline{\mathcal{C}}_{i}+\overline{\mathcal{C}}_{i+1}+\cdots+\overline{\mathcal{C}}_{n}\right) \\
& =\frac{1}{p^{n-i}} \sum_{a \in A_{i}} a-\frac{1}{p^{n-i+1}} \sum_{a \in A_{i-1}} a \\
& =\frac{1}{\left|A_{i}\right|} \sum_{a \in A_{i}} a-\frac{1}{\left|A_{i-1}\right|} \sum_{a \in A_{i-1}} a \\
& =\widetilde{A}_{i}-\widetilde{A}_{i-1} .
\end{aligned}
$$

Agora, consideremos o caso em que o grupo cíclico $G$ tem ordem $2 p^{n}$.

Mostraremos que, neste caso, os idempotentes primitivos encontrados no Teorema 6.1.6 são os mesmos encontrados por Arora e Pruthi [1].

Lembremos que $\mathbb{F}$ é o corpo finito com $q$ elementos, onde $q$ é uma potência de um primo ímpar tal que $m d c\left(p^{n}, q\right)=1$. Assumiremos que a ordem multiplicativa de $q$ módulo $2 p^{n}$ é $\phi\left(p^{n}\right), n \geq 1$. Então as $2 n+2$ q-classes ciclotômicas módulo $2 p^{n}$ são dadas por

$$
\begin{gathered}
\Omega_{i}=\left\{p^{i-1}, p^{i-1} q, \ldots, p^{i-1} q^{\rho\left(p^{n-\imath+1}\right)-1}\right\}, \\
\Omega_{i}^{*}=\left\{2 p^{i-1}, 2 p^{i-1} q, \ldots, 2 p^{i-1} q^{\phi\left(p^{n-i+1}\right)-1}\right\}
\end{gathered}
$$

onde $1 \leq i \leq n+1$ e $\Omega_{n+1}^{*}=\{0\}$. 
Note que os elementos de $\Omega_{i}$ são os números ímpares do conjunto $\left\{0,1,2, \ldots, 2 p^{n}-1\right\}$ divisíveis por $p^{i-1}$, mas não divisíveis por potências maiores de $p$ e os elementos de $\Omega_{i}^{*}$ são os números pares do conjunto $\left\{0,1,2, \ldots, 2 p^{n}-1\right\}$ divisíveis por $p^{i-1}$, mas não divisíveis por potências maiores de $p$.

Consideremos o anel $\mathcal{R}_{n}=\mathbb{F}[X] /\left\langle X^{2 n^{n}}-1\right\rangle$. Definimos os elementos $X_{i}(x), X_{i}^{*}(x)$ de $\mathcal{R}_{n}$ da seguinte forma

$$
X_{i}(x)=\sum_{s \in \Omega_{i}} x^{s} \quad \text { e } \quad X_{i}^{*}(x)=\sum_{s \in \Omega_{i}^{*}} x^{s}, \quad 1 \leq i \leq n+1 .
$$

Seja $G=\langle a\rangle$ um grupo cíclico de ordem $2 p^{n}$. Então $G$ se decompõe no produto direto de dois subgrupos cíclicos, digamos, $G=C \times A$, onde $C$ é o subgrupo cíclico gerado por $\left\langle a^{p^{n}}\right\rangle$ de ordem 2 e $A$ é o subgrupo cíclico gerado por $\left\langle a^{2}\right\rangle$ de ordem $p^{n}$.

Lembremos que os conjuntos $\Omega_{i}$ particionam os inteiros módulo $p^{n}$, para $1 \leq i \leq n+1$. Logo, cada elemento $h \in A$ pode ser escrito na forma

$$
h=a^{2 p^{i-1} q^{j}}, \quad 0 \leq j \leq \phi\left(p^{n-i+1}\right)-1,
$$

ou seja, o expoente de cada elemento de $A$ pertence ao conjunto $\Omega_{i}^{*}, 1 \leq i \leq$ $n+1$.

Assim, podemos reescrever o conjunto $\mathcal{S}_{h}$ da seguinte forma

$$
\mathcal{S}_{a^{2 p^{i-1}}}=\left\{a^{2 p^{i-1} q^{j}} \mid 0 \leq j \leq \phi\left(p^{n-i+1}\right)-1\right\} .
$$

Portanto, segue imediatamente que

$$
X_{i}^{*}(a)=\sum_{s \in \Omega_{i}^{*}} a^{s}=\sum_{h \in S} h .
$$

Agora, observemos que, se $a^{k} \in G$ tal que $o\left(a^{k}\right)=2 p^{n-i+1}$ então $m d c\left(k, 2 p^{n}\right)=$ $p^{i-1}$, o que implica que $p^{i-1} \mid k$. Logo, $k \in \Omega_{i}$ se $k$ for ímpar e $k \in \Omega_{i}^{*}$ se $k$ for par.

Vamos mostrar que

$$
X_{i}(a)=\sum_{s \in \Omega_{i}} a^{s}=a^{p^{n}} \sum_{h \in S^{\prime}, p^{p^{\prime}-1}} h .
$$


De fato, como

$$
X_{i}(a)=a^{p^{i-1}}+a^{p^{i-1} q}+\cdots a^{p^{i-1} q^{\phi\left(p^{n-i+1}\right)-1}}
$$

temos que cada termo dessa soma tem ordem $2 p^{n-i+1}$.

Agora,

$$
\begin{aligned}
a^{p^{n}} X_{i}^{*}(a) & =a^{p^{n}}\left(a^{2 p^{i-1}}+a^{2 p^{i-1} q}+\cdots a^{\left.2 p^{i-1} q^{\phi\left(p^{n-i+1}\right)-1}\right)}\right. \\
& =a^{2 p^{i-1}+p^{n}}+a^{2 p^{i-1} q+p^{n}}+\cdots a^{2 p^{i-1} q^{\phi\left(p^{n-i+1}\right)-1}+p^{n}} .
\end{aligned}
$$

Para cada termo da soma acima temos

$$
\left(a^{2 p^{i-1} q^{t}+p^{n}}\right)^{2 p^{n-i+1}}=\left(a^{2 p^{i-1} q^{t}}\right)^{2 p^{n-i+1}}\left(a^{p^{n}}\right)^{2 p^{n-i+1}}=1, \quad 0 \leq t \leq \phi\left(p^{n-i+1}\right)-1 .
$$

Agora, se $\alpha<n-i+1$ temos que $\alpha+i-1<n$ logo

$$
\left(a^{2 p^{i-1} q^{t}+p^{n}}\right)^{2 p^{\alpha}}=\left(a^{2 p^{i-1} q^{t}}\right)^{2 p^{\alpha}}\left(a^{p^{n}}\right)^{2 p^{\alpha}}=\left(a^{2 p^{\alpha+i-1}}\right)^{2 q^{t}} 1 \neq 1, \quad 0 \leq t \leq \phi\left(p^{n-i+1}\right)-1 .
$$

Portanto, a ordem de cada termo de $a^{p^{n}} X_{i}^{*}(a)$ é igual a $2 p^{n-i+1}$ e, como cada termo tem expoente ímpar, segue pela observação acima, que o expoente de cada termo da soma $a^{p^{n}} X_{i}^{*}(a)$ pertence a $\Omega_{i}$. Logo,

$$
X_{i}(a)=\sum_{s \in \Omega_{i}} a^{s}=a^{p^{n}} \sum_{h \in S_{a^{2 p^{i-1}}}} h .
$$

Agora, estamos em condições de mostrar que os idempotentes primitivos encontrados no Teorema 6.1.6 são os mesmos encontrados por Arora e Pruthi em [1]:

$$
\begin{gathered}
\varepsilon_{0}(x)=\frac{1}{2 p^{n}} \sum_{j=1}^{n+1}\left(X_{j}^{*}+X_{j}\right)(x), \\
\eta_{0}(x)=\frac{1}{2 p^{n}} \sum_{j=1}^{n+1}\left(X_{j}^{*}-X_{j}\right)(x), \\
\varepsilon_{i}(x)=\frac{1}{2 p^{n-i+1}}\left[(p-1) \sum_{j=i+1}^{n+1}\left(X_{j}^{*}+X_{j}\right)(x)-\left(X_{i}^{*}+X_{i}\right)(x)\right],
\end{gathered}
$$

e

$$
\eta_{i}(x)=\frac{1}{2 p^{n-i+1}}\left[(p-1) \sum_{j=i+1}^{n+1}\left(X_{j}^{*}-X_{j}\right)(x)-\left(X_{i}^{*}-X_{i}\right)(x)\right] \text {. }
$$


para $1 \leq i \leq n$.

Os idempotentes $\varepsilon_{0}$ e $\eta_{0}$ calculados em $a \in G$ nos dão

$$
\begin{aligned}
\varepsilon_{0}(a) & =\frac{1}{2 p^{n}}\left[\sum_{j=1}^{n+1} X_{j}^{*}(a)+\sum_{j=1}^{n+1} X_{j}(a)\right] \\
& =\frac{1}{2 p^{n}}\left[\sum_{j=1}^{n+1}\left(\sum_{h \in S_{a^{2} p^{j-1}}} h\right)+\sum_{j=1}^{n+1}\left(a^{p^{n}} \sum_{h \in S_{a^{2 p^{j-1}}}} h\right)\right] \\
& =\frac{1}{2 p^{n}}\left(\widehat{A}+a^{p^{n}} \widehat{A}\right) \\
& =\frac{1}{2} \widetilde{A}+\frac{a^{p^{n}}}{{ }^{2}} \widetilde{A} \\
& =\frac{\left(1+a^{p^{n}}\right)}{2} \widetilde{A}=\frac{\left(1+a^{p^{n}}\right)}{2} e_{0},
\end{aligned}
$$

e

$$
\begin{aligned}
\eta_{0}(a) & =\frac{1}{2 p^{n}}\left[\sum_{j=1}^{n+1} X_{j}^{*}(a)-\sum_{j=1}^{n+1} X_{j}(a)\right] \\
& =\frac{1}{2 p^{n}}\left[\sum_{j=1}^{n+1}\left(\sum_{h \in S_{a^{2 p^{j-1}}}} h\right)-\sum_{j=1}^{n+1}\left(a^{p^{n}} \sum_{h \in S_{a^{2 p^{j}-1}}} h\right)\right] \\
& =\frac{1}{2 p^{n}}\left(\widehat{A}-a^{p^{n}} \widehat{A}\right) \\
& =\frac{1}{2} \widetilde{A}-\frac{a^{p^{n}}}{2} \widetilde{A} \\
& =\frac{\left(1-a^{p^{n}}\right)}{2} \widetilde{A}=\frac{\left(1-a^{p^{n}}\right)}{2} e_{0} .
\end{aligned}
$$

Agora verificaremos os clemais elementos idempotentes $e_{i}$ e $\eta_{i}$. Para $1 \leq i \leq n$, temos

$$
\xi_{i}(x)=\frac{1}{2 p^{n-i+1}}\left[p \sum_{j=i+1}^{n+1}\left(X_{j}^{*}+X_{j}\right)(x)-\sum_{j=i}^{n+1}\left(X_{j}^{*}+X_{j}\right)(x)\right] .
$$

Portanto, calculando $\Xi_{i}(x)$ em $a \in G$ obtemos 


$$
\begin{aligned}
& \varepsilon_{i}(a)=\frac{1}{2 p^{n-i+1}}\left[p \sum_{j=i+1}^{n+1}\left(X_{j}^{*}+X_{j}\right)(a)-\sum_{j=i}^{n+1}\left(X_{j}^{*}+X_{j}\right)(a)\right] \\
& =\frac{1}{2 p^{n-i+1}}\left[p \sum_{j=i+1}^{n+1}\left(\sum_{h \in S_{a^{2} p^{j-1}}} h+a^{p^{n}} \sum_{h \in S_{a^{2} p^{j-1}}} h\right)+\right. \\
& \left.-\sum_{j=i}^{n+1}\left(\sum_{h \in S_{a^{2} p^{j-1}}} h+a^{p^{n}} \sum_{h \in S_{a^{2} p^{j-1}}} h\right)\right] \\
& =\frac{p}{2 p^{n-i+1}}\left[\widehat{A_{i}}+a^{p^{n}} \widehat{A_{i}}\right]-\frac{1}{2 p^{n-i+1}}\left[\widehat{A_{i-1}}+a^{p^{n}} \widehat{A_{i-1}}\right] \\
& =\frac{\widetilde{A_{i}}}{2}+a^{p^{n}} \frac{\widetilde{A_{i}}}{2}-\frac{\widetilde{A_{i-1}}}{2}-a^{p^{n}} \frac{\widetilde{A_{i-1}}}{2} \\
& =\frac{\left(\widetilde{A_{i}}-\widetilde{A_{i-1}}\right)}{2}+a^{p^{n}} \frac{\left(\widetilde{A_{i}}-\widetilde{A_{i-1}}\right)}{2} \\
& =\frac{e_{i}}{2}+a^{p^{n}} \frac{e_{i}}{2} \\
& =\frac{\left(1+a^{p^{n}}\right)}{2} e_{i}
\end{aligned}
$$

$\mathrm{e}$

$$
\eta_{i}(x)=\frac{1}{2 p^{n-i+1}}\left[p \sum_{j=i+1}^{n+1}\left(X_{j}^{*}-X_{j}\right)(x)-\sum_{j=i}^{n+1}\left(X_{j}^{*}-X_{j}\right)(x)\right]
$$

Calculanclo $\Sigma_{i}(x)$ em $a \in G$, obtemos 


$$
\begin{aligned}
& \eta_{i}(a)=\frac{1}{2 p^{n-i+1}}\left[p \sum_{j=i+1}^{n+1}\left(X_{j}^{*}-X_{j}\right)(a)-\sum_{j=i}^{n+1}\left(X_{j}^{*}-X_{j}\right)(a)\right] \\
& =\frac{1}{2 p^{n-i+1}}\left[p\left(\sum_{j=i+1}^{n+1} \sum_{h \in S_{\mathbf{a}^{2} p^{j-1}}} h-\sum_{j=i+1}^{n+1} a^{p^{n}} \sum_{h \in S_{a^{2} p^{j-1}}} h\right)+\right. \\
& \left.-\sum_{j=i}^{n+1}\left(\sum_{h \in S_{a^{2} p^{j-1}}} h-a^{p^{n}} \sum_{h \in S_{a^{2} p^{j-1}}} h\right)\right] \\
& =\frac{p}{2 p^{n-i+1}}\left[\widehat{A_{i}}-a^{p^{n}} \widehat{A_{i}}\right]-\frac{1}{2 p^{n-i+1}}\left[\widehat{A_{i-1}}-a^{p^{n}} \widehat{A_{i-1}}\right] \\
& =\frac{\widetilde{A_{i}}}{2}-a^{p^{n}} \frac{\widetilde{A_{i}}}{2}-\frac{\widetilde{A_{i-1}}}{2}+a^{p^{n}} \frac{\widetilde{A_{i-1}}}{2} \\
& =\frac{\left(\widetilde{A_{i}}-\widetilde{A_{i-1}}\right)}{2}-a^{p^{n}} \frac{\left(\widetilde{A_{i}}-\widetilde{A_{i-1}}\right)}{2} \\
& =\frac{e_{i}}{2}-a^{p^{n}} \frac{e_{i}}{2} \\
& =\frac{\left(1-a^{p^{n}}\right)}{2} e_{i} \text {. }
\end{aligned}
$$




\section{Capítulo 7}

\section{Códigos Abelianos Minimais}

Neste capítulo iremos estender os resultados apresentados no capítulo $V I$, ou seja, estenderemos para o caso em que os grupos em questão são abelianos finitos. Continuaremos assumindo que $\mathbb{F}$ é um corpo finito com $|\mathbb{F}|=q$ elementos e $q$ é uma potência de um primo tal que $m d c(q,|A|)=1$, onde $|A|$ denota a ordem do grupo abeliano $A$.

\subsection{O Cálculo dos Idempotentes}

Primeiramente consideremos o caso de $p$-grupos.

Seja $A$ um $p$-grupo abeliano. Para cada subgrupo $H$ de $A$ tal que $\{1\} \neq$ $A / H$ é cíclico nos iremos construir um idempotente de $\mathbb{F} A$.

Relembrando: desde que $A / H$ é um grupo cíclico de ordem igual a uma potência de um primo $p, H \neq A$, existe apenas um subgrupo $H^{*}$ de $A$ contendo $H$ tal que $\left|H^{*} / H\right|=p$.

Definimos $e_{H}=\widetilde{H}-\widetilde{H^{*}}$. Claramente $e_{H} \neq 0$ e temos o seguinte resultado.

Lema 7.1.1. Os elementos $e_{H}$ definidos acima, junto com $e_{A}=\tilde{A}$, formam um conjunto de idempotentes, dois a dois ortogonais, de $\mathbb{F} A$ cuja soma é igual a 1 .

Demonstração: Primeiramente vamos mostrar que estes elementos são idempotentes.

Temos que

$$
e_{A} e_{A}=\tilde{A} \widetilde{A}=\left(\frac{1}{|A|} \widehat{A}\right)\left(\frac{1}{|A|} \widehat{A}\right)=\frac{1}{|A||A|} \widehat{A} \hat{A}=\frac{1}{|A|} \cdot \hat{A}=e_{A} .
$$


e

$$
\begin{aligned}
e_{H} e_{H} & =\left(\widetilde{H}-\widetilde{H^{*}}\right)\left(\widetilde{H}-\widetilde{H^{*}}\right) \\
& =\widetilde{H} \widetilde{H}-\widetilde{H} \widetilde{H^{*}}-\widetilde{H^{*}} \widetilde{H}+\widetilde{H^{*}} \widetilde{H^{*}} .
\end{aligned}
$$

Como $H^{*} \supset H$ segue que

$$
\begin{aligned}
e_{H} e_{H} & =\widetilde{H}-\widetilde{H^{*}}-\widetilde{H^{*}}+\widetilde{H^{*}} \\
& =\widetilde{H}-\widetilde{H^{*}}=e_{H} .
\end{aligned}
$$

Sejam $H$ e $K$ subgrupos distintos de $A$ tal que $A / H$ e $A / K$ são ambos cíclicos, diferentes de $\{1\}$ e, sejam $H^{*}$ e $K^{*}$ subgrupos de $A$ contendo $H$ e $K$, respectivamente, tal que $H^{*} / H$ e $K^{*} / K$ são ambos cíclicos de ordem $p$.

Consideremos primeiro o caso em que $H \subset K$.

Tem-se que $|K / H|=p^{j}, j \geq 1$. Logo, existe um subgrupo, $\{1\} \neq H_{1}$ de $K$ tal que $H \subset H_{1} \subset K$ e $\left|H_{1} / H\right|=p$. Como $H \subset H_{1} \subset K \subset A$ segue que, $H_{1}=H^{*}$, pela unicidade de $H^{*}$.

Assim, $H^{*} \subseteq K$.

Portanto,

$$
\begin{aligned}
e_{H} e_{K} & =\left(\widetilde{H}-\widetilde{H^{*}}\right)\left(\widetilde{K}-\widetilde{K^{*}}\right) \\
& =\widetilde{H} \widetilde{K}-\widetilde{H} \widetilde{K^{*}}-\widetilde{H^{*}} \widetilde{K}+\widetilde{H^{*}} \widetilde{K^{*}} \\
& =\widetilde{K}-\widetilde{K^{*}}-\widetilde{K}+\widetilde{K^{*}}=0 .
\end{aligned}
$$

Agora, se nenhum desses subgrupos estiver contido no outro então $H$ e $K$ estão contidos propriamente em $H K$.

Assim, $H \subset H K$. Tem-se que $|H K / H|=p^{i}, i \geq 1$. Logo, existe um subgrupo, $\{1\} \neq H_{2}$ de $H K$ tal que $H \subset H_{2} \subset H K \subset A$ e $\left|H_{2} / H\right|=p$. Pela unicidade de $H^{*}$, temos que $H_{2}=H^{*}$.

Portanto, $H^{*} \subset H K$.

Analogamente, tem-se que $K^{*} \subset H K$.

Portanto, $H^{*} K^{*} \subset H K$ e, como $H K \subset H^{*} K^{*}$, segue que $H K=H^{*} K^{*}$.

Descle que $H K \subset H K^{*} \subset H^{*} K^{*}$ scgue também que $H K^{*}=H K$ e, da mesma forma $H K \subset H^{*} K \subset H^{*} K^{*}=H K \log 0 H^{*} K=H K$.

Portanto,

$$
\begin{aligned}
e_{H} e_{K} & =\left(\widetilde{H}-\widetilde{H^{*}}\right)\left(\widetilde{K}-\widetilde{K^{*}}\right) \\
& =\widetilde{H} \widetilde{K}-\widetilde{H} \widetilde{K^{*}}-\widetilde{H^{*}} \widetilde{K}+\widetilde{H^{*}} \widetilde{K^{*}} \\
& =\widetilde{H} \widetilde{K}-\widetilde{H} \widetilde{K}-\widetilde{H} \tilde{K}+\widetilde{H} \widetilde{K}=0 .
\end{aligned}
$$


Agora, se um dos idempotentes é igual a $e_{A}$ tem-se que

$$
e_{H} e_{A}=\left(\widetilde{H}-\widetilde{H^{*}}\right) \widetilde{A}=\widetilde{H} \widetilde{A}-\widetilde{H^{*}} \widetilde{A}=\widetilde{A}-\widetilde{A}=0
$$

Assim, estes são os idempotentes ortogonais de $\mathbb{F} A$.

Finalmente, iremos mostrar que a soma deles é igual a 1.

Para cada subgrupo cíclico $C$ se $A$ denotaremos por $\mathcal{G}(C)$ o conjunto de todos elementos de $C$ que geram o subgrupo, isto é,

$$
\mathcal{G}(C)=\{c \in C \mid m d c(o(c),|C|)=1\} .
$$

Se $\mathcal{C}$ denota a família de todos os subgrupos cíclicos de $A$ então

$$
|A|=\sum_{C \in \mathcal{C}}|\mathcal{G}(C)| .
$$

Se $C$ é um grupo cíclico de uma dada ordem $p^{r}$, temos que $\left|\mathcal{G}\left(C^{\prime}\right)\right|=$ $\phi\left(p^{r}\right)=p^{r}-p^{r-1}=|C|-|C| / p$.

Seja $\mathcal{S}$ o conjunto de todos os subgrupos $H$ de $A$ tal que o quociente $A / H$ é cíclico. Denotaremos $e=\sum_{H \in \mathcal{S}} e_{H}$.

Afirmamos que $e=1$.

Para provarmos isto é suficiente mostrarmos que $(\mathbb{F} A) e=\mathbb{F} A$.

Conı mostramos anteriormente, os idempotentes que compõem $e$ são ortogonais dois a dois, logo temos que

$$
(\mathbb{F} A) e=\bigoplus_{H \in \mathcal{S}}(\mathbb{F} A) e_{H}
$$

então

$$
\operatorname{dim}_{\mathbb{F}}((\mathbb{F} A) e)=\sum_{H \in \mathcal{S}} \operatorname{dim}_{\mathbb{F}}\left((\mathbb{F} A) e_{H}\right) .
$$

Note que $\widetilde{H}=\widetilde{H^{*}}+e_{H}$ e que $\widetilde{H^{*}} e_{H}=0$.

Portanto,

$$
(\mathbb{F} A) \widetilde{H}=(\mathbb{F} A) \widetilde{H^{*}} \oplus(\mathbb{F} A) e_{H} .
$$

Assim,

$$
\operatorname{dim}_{\mathbb{F}}\left((\mathbb{F} A) e_{H}\right)=\operatorname{dim}_{\mathbb{F}}((\mathbb{F} A) \widetilde{H})-\operatorname{dim}_{F}\left((\mathbb{F} A) \widetilde{H^{*}}\right) .
$$


Segue da demonstração da Proposição 1.4 .13 que

$$
\operatorname{dim}_{\mathbb{F}}\left((\mathbb{F} A) e_{H}\right)=\operatorname{dim}_{\mathbb{F}}\left((\mathbb{F}(A / H))-\operatorname{dim}_{\mathbb{F}}\left(\left(\mathbb{F}\left(A / H^{*}\right)\right)\right.\right.
$$

e temos que

$$
\operatorname{dim}_{\mathbb{F}}\left(( \mathbb { F } ( A / H ) ) = | A / H | \quad \text { e } \quad \operatorname { d i m } _ { \mathbb { F } } \left(\left(\mathbb{F}\left(A / H^{*}\right)\right)=\left|A / H^{*}\right| .\right.\right.
$$

Do Teorema 1.6.6 segue que existe uma bijeção $\Phi: \mathcal{C} \longrightarrow \mathcal{S}$ tal que $|X|=|A / \Phi(X)|$, para todo $X \in \mathcal{C}$.

Se denotarmos por $C \in \mathcal{C}$ o subgrupo tal que $\Phi(C)=H$ temos que

$$
\operatorname{dim}_{\mathbb{F}}((\mathbb{F}(A / H))=|A / H|=|C|
$$

$\mathrm{e}$

$$
\operatorname{dim}_{\mathbb{F}}\left(\left(\mathbb{F}\left(A / H^{*}\right)\right)=\left|A / H^{*}\right|=|A / H| /\left|H^{*} / H\right|=|C| / p .\right.
$$

Assim,

$$
\operatorname{dim}_{\mathbb{F}}\left((\mathbb{F} A) e_{H}\right)=|C|-|C| / p=|\mathcal{G}(C)|
$$

e portanto,

$$
\sum_{H \in \mathcal{S}} \operatorname{dim}_{\mathbb{F}}\left((\mathbb{F} A) e_{H}\right)=\sum_{C \in \mathcal{C}}|\mathcal{G}(C)|=|A|
$$

e segue o resultado, uma vez que

$$
\operatorname{dim}_{\mathbb{F}}((\mathbb{F} A) e)=|A|=\operatorname{dim}_{\mathbb{F}}(\mathbb{F} A) .
$$

O próximo resultado é consequência imediata do Lema 7.1.1 e do Corolário 2.2.3.

Teorema 7.1.2. Sejam p um primo ímpar e A um p-grupo abeliano de expoente $p^{r}$. Então, o conjunto de idempotentes do Lema 7.1.1 é o conjunto de idempotentes primitivos de $\mathbb{F} A$ se e somente se vale uma das seguintes afirmações:

(i) $p=2$ eq é ímpar;

(ii) $p^{r}=4 e q \equiv 3 \quad(\bmod 4)$; 
(iii) se p é um primo ímpar e o $(\bar{q})=\phi\left(p^{n}\right)$ em $U\left(\mathbb{Z}_{p^{n}}\right)$.

Demonstração: Consideremos o conjunto de idempotentes de $\mathbb{F} A$ dado pelo Lema 7.1.1. Se estes são os idempotentes primitivos de $\mathbb{F} A$ então o número de componentes simples de $\mathbb{F} A$ é igual ao número de fatores cíclicos de $A$. Do Teorema 1.4 .8 segue que o número de fatores cíclicos de $A$ é igual ao número de componentes simples de $\mathbb{Q} A$, o que implica que $\mathcal{S}_{g}=\mathcal{G}_{g}$. Assim, do Corolário 2.2.3 valem as condições do enunciado.

Reciprocamente, os idempotentes de $\mathbb{F} A$ dado pelo Lema 7.1 .1 são ortogonais dois a dois e somam 1. Tem-se que o número destes idempotentes é igual ao número de quocientes cíclicos de $\mathrm{A}$, que é igual ao número de subgrupos cíclicos de $A$. Do Teorema 1.4 .8 segue que o número de fatores cíclicos de $A$ é igual ao número de componentes simples de $\mathbb{Q} A$. Do Corolário 2.2.3 segue que o número de componentes simples de $\mathbb{F} A$ é igual ao de $\mathbb{Q} A$.

Portanto, este é o conjunto de idempotentes primitivos de $\mathbb{F} A$. De fato, se pudermos escrever $e_{i}=e_{i}^{\prime}+e_{i}^{\prime \prime}$, para algum índice $i$, onde $e_{i}^{\prime}, e_{i}^{\prime \prime}$ são idempotentes ortogonais, não nulos, teríamos um conjunto com um número maior de idempotentes ortogonais cuja soma desses é igual a 1, ou seja, teríamos um número maior de componentes simples de $\mathbb{F} A$, uma contradição.

Também temos o seguinte resultado:

Teorema 7.1.3. Sejam $p$ um primo ímpar e $A$ um p-grupo abeliano de expoente $2 p^{r}$. Escrevendo $A=E \times B$, onde $E$ é um 2-grupo abeliano elementar $e B$ é um p-grupo. Então os idempotentes primitivos de $\mathbb{F} A$ são produtos da forma $e \cdot f$, onde e é um idempotente primitivo de $\mathbb{F} E$ e $f$ um idempotente primitivo de $\mathbb{F} B$.

Demonstração: Como os idempotentes primitivos de $\mathbb{F} B$ são dados pelo Teorema 7.1.2 e, escrevendo $E=\left\langle a_{1}\right\rangle \times\left\langle a_{2}\right\rangle \times \cdots \times\left\langle a_{m}\right\rangle$ como produto de grupos cíclicos de ordem 2 , temos que os idempotentes primitivos de $\mathbb{F} E$ são todos os produtos da forma $e=e_{1} e_{2} \cdots e_{m}$ onde

$$
e_{i}=\frac{1+a_{i}}{2} \quad \text { ou } \quad e_{i}=\frac{1-a_{i}}{2}, \quad 1 \leq i \leq m
$$

Logo, segue da demonstração do Teorema 6.1.6 que os idempotentes primitivos de $\mathbb{F} A$ são produtos da forma $e \cdot f$, onde $e$ é um idempotente primitivo de $\mathbb{F} E$ e $f$ um idempotente primitivo de $\mathbb{F} B$. 


\subsection{Dimensão e distância mínima}

A partir de agora, assumiremos que $|A|=2^{m} p^{n}$, onde $p$ denota um primo ímpar, $m \geq 0$ e $p$ é tal que $o(q)=\phi\left(p^{n}\right)$ em $U\left(\mathbb{Z}_{p^{n}}\right)$.

Assim, podemos escrever $A=E \times B$, onde $E$ é um 2-grupo abeliano elementar de ordem $2^{m}$ (eventualmente trivial) e $B$ é um $p$-grupo.

Note que, no Teorema 7.1.2 da seção anterior, encontramos os idempotentes primitivos de $\mathbb{F} B$. Agora, escrevendo $E=\left\langle a_{1}\right\rangle \times\left\langle a_{2}\right\rangle \times \cdots \times\left\langle a_{m}\right\rangle$ como produto de grupos cíclicos de ordem 2 , temos que os idempotentes primitivos de $\mathbb{F} E$ são todos os produtos da forma $e=e_{1} e_{2} \cdots e_{m}$ onde

$$
e_{i}=\frac{1+a_{i}}{2} \quad \text { oul } \quad e_{i}=\frac{1-a_{i}}{2}, \quad 1 \leq i \leq m
$$

Logo, os idempotentes primitivos de $\mathbb{F} A$ são produtos da forma $e_{E} e_{B}$, onde $e_{E}$ é um idempotente primitivo de $\mathbb{F} E$ e $e_{B}$ um idempotente primitivo de $\mathbb{F} B$.

Agora, para idempotentes fixos $e_{E}$ de $\mathbb{F} E$ e um elemento $y \in E$, podemos escrever $y=a_{1}^{\varepsilon_{1}} a_{2}^{\varepsilon_{2}} \cdots a_{m}^{\varepsilon_{m}}$, onde $\varepsilon_{i}=0$ ou $1,1 \leq i \leq m$.

Note que, se $\varepsilon_{i}=1$ então $a_{i}^{\varepsilon_{i}} a_{i}=a_{i}^{2}=1$, pois $E$ é um 2-grupo abeliano elementar de ordem $2^{m}$, donde $a_{i}^{\varepsilon_{i}}\left(\frac{1 \pm a_{i}}{2}\right)=-\left(\frac{1 \pm a_{i}}{2}\right)$.

Portanto,

$$
\begin{aligned}
y e_{E} & =a_{1}^{\varepsilon_{1}} a_{2}^{\varepsilon_{2}} \cdots a_{m}^{\varepsilon_{m}}\left(\frac{1 \pm a_{1}}{2}\right)\left(\frac{1 \pm a_{2}}{2}\right) \cdots\left(\frac{1 \pm a_{m}}{2}\right) \\
& =a_{1}^{\varepsilon_{1}}\left(\frac{1 \pm a_{1}}{2}\right) a_{2}^{\varepsilon_{2}}\left(\frac{1 \pm a_{2}}{2}\right) \cdots a_{m}^{\varepsilon_{m}}\left(\frac{1 \pm a_{m}}{2}\right) \\
& = \pm e_{E}=(-1)^{\varepsilon_{y}} e_{E}
\end{aligned}
$$

onde $\varepsilon_{y}=0$ ou 1 .

Vamos considerar primeiro os idempotentes primitivos da forma $e_{E} \widetilde{B}$, onde $\widetilde{B}$ é o idempotente principal de $\mathbb{F} B$.

Um elemento de $(\mathbb{F} A) e_{E} \widetilde{B}$ é da forma $\gamma e_{E} \widetilde{B}$ onde podemos escrever $\gamma=$ $\sum_{\substack{y \in E \\ b \in B}} x_{y b} y b$. Então, temos que 


$$
\begin{aligned}
\gamma \cdot e_{E} \widetilde{B} & =\sum_{\substack{y \in E \\
b \in B}} x_{y b} y b \cdot e_{E} \widetilde{B} \\
& =\sum_{\substack{y \in E \\
b \in B}} x_{y b} y e_{E} \cdot b \widetilde{B} \\
& =\sum_{\substack{y \in E \\
b \in B}} x_{y b}(-1)^{\varepsilon_{y}} e_{E} \cdot b \widetilde{B} \\
& =\left(\sum_{\substack{y \in E \\
b \in B}} x_{y b}(-1)^{\varepsilon_{y}}\right) e_{E} \widetilde{B} .
\end{aligned}
$$

Note que, $\sum_{\substack{y \in E \\ b \in B}} x_{y b}(-1)^{\varepsilon_{y}} \in \mathbb{F}$.

O cálculo acima nos mostra que $(\mathbb{F} A) e_{B} \widetilde{B}=\mathbb{F} e_{B} \widetilde{B}$, logo a dimensão do ideal $\mathcal{I}=(\mathbb{F} A) e_{E} \widetilde{B}$ é igual a 1 .

Tem-se que

$$
e_{E} \widetilde{B}=\left(\frac{1 \pm a_{1}}{2}\right)\left(\frac{1 \pm a_{2}}{2}\right) \cdots\left(\frac{1 \pm a_{m}}{2}\right) \sum_{b \in B} b .
$$

Portanto,

$$
e_{E} \widetilde{B}=\left(\sum_{x \in E} \delta(x) x\right) \sum_{b \in B} b
$$

onde $\delta(x)=1$ ou -1 .

Logo,

$$
e_{E} \widetilde{B}=\left(\sum_{a \in A} \sigma(a) a\right)
$$

onde $\sigma(a)=1$ ou -1 .

Assim, $d\left(e_{E} \widetilde{B}\right)=|A|$ e, como a dimensão do ideal $\mathcal{I}=(\mathbb{F} A) e_{E} \widetilde{B}$ é igual a 1 , todo outro elemento é um inultiplo escalar de $\epsilon_{E} \widetilde{B}$. Portanto, segue que $d(\mathcal{I})=|A|$.

Agora, consideremos os idempotentes primitivos da forma $e=e_{E} e_{H}$, onde $e_{E} \in \mathbb{F} E$ como acima e $e_{H}=\widetilde{H}-\widetilde{H^{*}}$, onde $H$ ć um subgrupo de $B$ tal que $B / H$ é cíclico de ordem $p^{i}$, e $H^{*}$ é o ínico subgrupo de $B$ contendo $H$ tal que $\left[H^{*}: H\right]=p$. Seja $\mathcal{I}_{e}=(\mathbb{F} A) e$. 
Seja $b \in B$ um elemento tal que $B=\langle b, H\rangle$. Então, temos também que $H^{*}=\left\langle b^{p^{i-1}}, H\right\rangle$. Note que

$$
\begin{aligned}
\left(1-b^{p^{i-1}}\right) e_{E} \widetilde{H} & =\left(1-b^{p^{i-1}}\right) e_{E}\left(e_{H}+\widetilde{H^{*}}\right) \\
& =\left(1-b^{p^{i-1}}\right) e_{E} e_{H}+\left(1-b^{p^{i-1}}\right) e_{E} \widetilde{H^{*}} \\
& =\left(1-b^{p^{i-1}}\right) e_{E} e_{H}+e_{E} \widetilde{H^{*}}-b^{p^{i-1}} e_{E} \widetilde{H^{*}} \\
& =\left(1-b^{p^{i-1}}\right) e_{E} e_{H}+e_{E} \widetilde{H^{*}}-e_{E} \widetilde{H^{*}} \\
& =\left(1-b^{p^{i-1}}\right) e_{E} e_{H} \in \mathcal{I}_{e} .
\end{aligned}
$$

Desde que, $b^{p^{i-1}} \notin H$ é claro que $\operatorname{supp}\left(\left(1-b^{p^{i-1}}\right) \widetilde{H}\right)$ é a união disjunta de $H \cup b^{p^{i-1}} H$. Assim, o peso do elemento $\left(1-b^{p^{i-1}}\right) \widetilde{H}$ é igual a $2|H|$, donde o peso do elemento $\left(1-b^{p^{i-1}}\right) e_{E} \widetilde{H}$ é igual a $2|E||H|$.

Se denotarmos por $d\left(\mathcal{I}_{e}\right)$ a distância minima de $\mathcal{I}_{e}$ temos que

$$
d\left(\mathcal{I}_{e}\right) \leq 2^{m+1}|H|
$$

Descle que $B$ é a união disjunta de $H \cup b H \cup \cdots \cup b^{p^{i}-1} H$ segue que $A=E \times B$ pode ser escrito como

$$
\begin{aligned}
A & =E \times\left(H \cup b H \cup \cdots \cup b^{p^{i}-1} H\right) \\
& =E \times H \cup b(E \times H) \cup \cdots \cup b^{p^{i}-1}(E \times H) .
\end{aligned}
$$

Então um elemento arbitrário de $\mathbb{F} A$ pode ser escrito na forma

$$
\alpha=\sum_{j=0}^{p^{i}-1} \alpha_{j} b^{j}, \quad \text { com } \quad \alpha_{j} \in \mathbb{F}(E \times H) .
$$

Agora, levando em conta a fórmula (2) e o fato que $h \widetilde{H}=\widetilde{H}$, para todo $h \in H$. Se $\alpha_{j} \in \mathbb{F}(E \times H)$ então $\alpha_{j}=\sum_{\substack{i, \in E \\ h \in H}} \alpha_{y h} y h$. Assim,

$$
\begin{aligned}
\alpha_{j} \cdot e_{E} e_{H} & =\sum_{\substack{y \in E \\
h \in H}} \alpha_{y, h} y h \cdot e_{E} e_{H} \\
& =\sum_{\substack{y \in E \\
h \in H}} \alpha_{y h} y e_{E} \cdot h e_{H} \\
& =\sum_{\substack{y \in E \\
h \in H}} \alpha_{y h}(-1)^{\bar{z}_{y}} e_{E} e_{I I} .
\end{aligned}
$$


Tomando $k_{j}=\sum_{\substack{y \in E \\ h \in H}} \alpha_{y h}(-1)^{\varepsilon_{y}} \in \mathbb{F}$ temos que cada produto $\alpha_{j} e_{E} e_{H}$ é da forma $\alpha_{j} e_{E} e_{H}=k_{j} e_{E} e_{H}$, onde $k_{j} \in \mathbb{F}, 0 \leq j \leq p^{i}-1$.

Como $(\mathbb{F} A) \cdot e_{E} e_{H} \subset(\mathbb{F} A) \cdot e_{E} \widetilde{H}$, um elemento $0 \neq \gamma \in(\mathbb{F} A) e_{E} e_{H}=\mathcal{I}_{e}$ pode ser escrito na forma

$$
\gamma=\alpha e_{E} \widetilde{H}=\left(k_{0}+k_{1} b+\cdots+k_{p^{i}-1} b^{p^{i}-1}\right) e_{E} \widetilde{H} .
$$

Desde que $\gamma \neq 0$ temos que no mínimo um coeficiente $k_{j} \neq 0$. Se $\gamma=$ $k_{j} b^{j} e_{E} \widetilde{H}$ nos temos que $e_{E} \widetilde{H} \in(\mathbb{F} A) e_{E} e_{H}$, uma contradição.

Portanto, pelo menos dois coeficientes $k_{j}, k_{l}$ devem ser não nulos para cada $\gamma \in \mathcal{I}_{e}$ e, assim $d\left(\mathcal{I}_{e}\right) \geq 2^{m+1}|H|$.

Portanto,

$$
d\left(\mathcal{I}_{e}\right)=2^{m+1}|H| .
$$

Finalmente, podemos calcular a dimensão dos ideais minimais.

Seja $e=e_{E} e_{H}$ um idempotente primitivo de $\mathbb{F} A$. Temos:

$\mathbb{F} A \cdot e_{E} e_{H}=\mathbb{F}(E \times B) \cdot e_{E} e_{H}=((\mathbb{F} E) B) \cdot e_{E} e_{H}=\left(\mathbb{F} E e_{E}\right) B e_{H}$.

Como $(\mathbb{F} E) e_{E} \cong \mathbb{F}$ para todo idempotente primitivo de $\mathbb{F} E$ temos que,

$$
\mathbb{F} A \cdot e_{E} e_{H} \cong \mathbb{F} B e_{H} .
$$

Assim, a fórmula (1) nos dá

$$
\begin{aligned}
\operatorname{dim}_{\mathbb{F}}\left(\mathbb{F} B e_{H}\right) & =\operatorname{dim}_{\mathbb{F}}(\mathbb{F}(B / H))-\operatorname{dim}_{\mathbb{F}}\left(\mathbb{F}\left(B / H^{*}\right)\right) \\
& =|B / H|-\left|B / H^{*}\right| \\
& =p^{i}-p^{i-1}
\end{aligned}
$$

isto é,

$$
\operatorname{dim}_{\mathbb{F}}\left(\mathbb{F} A e_{E} e_{H}\right)=\operatorname{dim}_{\mathbb{F}}\left(\mathbb{F} B e_{H}\right)=\phi\left(p^{i}\right) .
$$

Analogamente, temos que

$$
\operatorname{dim}_{\mathbb{F}}\left(\mathbb{F} A e_{E} \widetilde{B}\right)=\operatorname{dim}_{\mathbb{F}}(\mathbb{F} B \cdot \widetilde{B})=1 .
$$

De fato, como

$$
\mathbb{F} A \cdot e_{E} \widetilde{B}=\mathbb{F}(E \times B) \cdot e_{E} \widetilde{B}=((\mathbb{F} E) B) e_{E} \widetilde{B}=\left(\mathbb{F} E e_{E}\right) B \widetilde{B} .
$$

Como $\mathbb{F} E e_{E} \cong \mathbb{F}$ para todo idempotente primitivo de $\mathbb{F} E$ temos que

$$
\mathbb{F} A \cdot e_{E} \widetilde{B} \cong \mathbb{F} B \widetilde{B} \text {. }
$$

Assim.

$$
\operatorname{dim}_{\mathbb{F}}\left(\mathbb{F} A \cdot e_{E} \widetilde{B}\right)=\operatorname{dim}_{\mathbb{F}}(\mathbb{F} B \widetilde{B})=1
$$




\section{Referências Bibliográficas}

[1] S. K. Arora and M. Pruthi, Minimal cyclic codes of length $2 p^{n}$, Finite Field and Appl., 5(1999), 177-187.

[2] I. F. Blake and R.C. Mullin, The Mathematical Theory of Coding, Academic Press, New York, 1975.

[3] C. W. Curtis and I. Reiner, Methods of Representation Theory, vol. I, Wiley Interscience, New York, 1981.

[4] A. Hefez e M. L. T. Villela, Códigos Corretores de Erros, Rio de Janeiro, IMPA, 2002.

[5] L. Dornhoff, Group Representation Theory, Part B, Marcel Dekker Inc., New York, 1971.

[6] R. Ferraz, Simple components and central units in group algebras, J. Algebra, 279 (2004), 191-203.

[7] R. Ferraz and C. Polcino Milies, Idempotents in Group and Minimal Abelian Codes, preprint, 2004.

[8] E. G. Goodaire, E. Jespers and C. Polcino Milies, Alternative Loop Rings, North-Holland Math. Studies No. 184, Elsevier, Amsterdam, 1996.

[9] R. W. Hamming, Interview, February 3 - 4, 1977.

[10] R. W. Hamming, Error Detecting and Error Correcting Codes, The Bell. System Technical J., XXVI (1948), 379 - 423, 623 - 656.

[11] A. V. Kelarev and P. Solé, Error-correcting cocles as ideals in group rings, Contemp. Math., 273 (2001), 11-18. 
[12] B. Khülshammer, Bemerkugen über die Gruppenalgebra als symmetrische Albegra III, J. of Algebra, 88 (1984), 279 - 291.

[13] R. Lidl and H. Niederreiter, Finite Fields, second edition, Cambridge University Press, 1997.

[14] F. J. MacWilliams and N. J. A. Sloane, The Theory of Error-Correcting Codes, The Mathematical Association of America, vol. 21, 1983.

[15] P. A. Martin, Introdução à Teoria dos Grupos e à Teoria de Galois, São Paulo, IME-USP, 1998.

[16] V. S. Pless and W. C. Huffman, Handbook of Coding Theory, vol. I, Elsevier Amsterdam Lausanne New York Oxford Shannon Singapore Tokyo, 1998.

[17] C. Polcino Milies, Anéis de Grupo, IV Esc. de Álgebra, São Paulo, 1976.

[18] C. Polcino Milies and S.K. Sehgal, An Introduction to Group Rings, Kluwer Academic Publishers, Dordrecht, 2002.

[19] M. Pruthi and S.K. Arora, Minimal codes of prime power length, Finite Field and Appl., 3(1997), 93-113.

[20] C. E. Shannon, A Mathematical Theory of communication, The Bell System Technical Journal, vol. XXVIII, april, 1950, n.o 2.

[21] S. Roman, Introduction to Coding and InformationTheory, SpringerVerlang New York Berlin Heidelberg, 1996.

[22] S. Roman, Coding and Information Theory, Springer-Verlang New York Berlin Heidelberg, 1992.

[23] T. M. Thompson, From Error-Correcting Codes Through Sphere Packings To Simple Groups, North-Holland Publishing Company, 1977.

[24] J. H. van Lint, An Introduction to Coding Theory, Springer-Verlang New York Inc., 1982.

[25] G. Neuberger, F. de Lima, L. Carro e R. Reis, Projeto de uma Memória SRAM Tolerante a Múltiplas Falhas, R. Elet. Inic. Cient., 3, 3 (2003). 
[26] www.microprocessadores.hpg.ig.com.br

[27] www.jpl.nasa.gov 